University of Rhode Island

DigitalCommons@URI

Open Access Dissertations

2020

\title{
OPTICAL PROPERTIES OF ONE DIMENSIONAL QUATERNARY PHOTONIC CRYSTALS AND ASSOCIATED HETEROSTRUCTURES
}

Nicholas J. Bianchi

University of Rhode Island, nicholasbianchi@uri.edu

Follow this and additional works at: https://digitalcommons.uri.edu/oa_diss

\section{Recommended Citation}

Bianchi, Nicholas J., "OPTICAL PROPERTIES OF ONE DIMENSIONAL QUATERNARY PHOTONIC CRYSTALS AND ASSOCIATED HETEROSTRUCTURES" (2020). Open Access Dissertations. Paper 1146.

https://digitalcommons.uri.edu/oa_diss/1146

This Dissertation is brought to you for free and open access by DigitalCommons@URI. It has been accepted for inclusion in Open Access Dissertations by an authorized administrator of DigitalCommons@URI. For more information, please contact digitalcommons-group@uri.edu. 
OPTICAL PROPERTIES OF ONE DIMENSIONAL QUATERNARY PHOTONIC CRYSTALS AND ASSOCIATED HETEROSTRUCTURES

BY

NICHOLAS J. BIANCHI

A DISSERTATION SUBMITTED IN PARTIAL FULFILLMENT OF THE REQUIREMENTS FOR THE DEGREE OF

DOCTOR OF PHILOSOPHY

IN

PHYSICS

UNIVERSITY OF RHODE ISLAND

2020 
DOCTOR OF PHILOSOPHY DISSERTATION

$\mathrm{OF}$

NICHOLAS J. BIANCHI

\title{
APPROVED:
}

Dissertation Committee:

\author{
Major Professor Leonard Kahn \\ Alexander Meyerovich \\ Orlando Merino \\ Nasser H. Zawia \\ DEAN OF THE GRADUATE SCHOOL
}

\section{UNIVERSITY OF RHODE ISLAND}




\begin{abstract}
The research conducted in this dissertation is the theoretical investigation into the transmission properties of one dimensional inversion symmetric quaternary photonic crystals and heterostructures created by combining quaternary and binary crystals. A photonic crystal is a device constructed from dielectric or conducting scattering elements arranged in a periodic manner. Saying a crystal has inversion symmetry simply means that the individual unit cells do too. Inversion symmetry is needed to ensure the system remains reciprocal and that topological phase of bands in a band structure remains discrete. The terms "binary" and "quaternary" refer to the number of layers in a unit cell, in this case, two and four, respectively. Similar to how an ionic lattice manipulates the flow of electrons, the periodicity of a photonic crystal can control the flow of photons. This leads to interesting physical properties in a device, such as photonic band gaps, regions in frequency space where photonic states cannot exist, and interface modes, localized states that can form at the boundary between two different crystals due to some change in material parameters or geometry.

In the first manuscript, we investigate interface modes in a heterostructure composed of a single binary photonic crystal and single quaternary photonic crystal. Several papers have already investigated modes in structures solely consisting of binary crystals. Being one dimensional, quaternary crystals are still relatively simple to fabricate while providing more interesting behavior compared with a binary system. In the work, this is done by inserting a tunable layer in between every original layer of the binary crystals. By setting other parameters in the heterostructure constant, it is possible to smoothly transform one binary photonic crystal into another through an intermediate quaternary state. The transfer matrix method is used in the simulation of these crystals and their resultant prop-
\end{abstract}


erties. Identifying topological phase in the band structure for quaternary crystals is discussed and compared to that of binary crystals. Band gap closings are also discussed. Examples of topological interface modes in the transmission spectra of binary/quaternary structures are provided showing that modes only exist in certain band gap when the quaternary crystal is tuned from one binary to another.

In the second manuscript, the single binary/quaternary system mentioned previously is generalized to a periodic array of crystals with each crystal still being periodic itself. Interface modes are shown to display different behavior as the tunable layer in the quaternary crystal(s) increases. Topological modes in the band gap are shown to vanish, split in two, or be surrounded by additional modes. Investigations are done for systems consisting entirely of lossless dielectrics and for systems where the tunable layer has a frequency dependent refractive index via the Drude model. Also, examples are given of how modes couple as the number of unit cells in the central crystal of a heterostructure changes.

In the third manuscript, effective medium theory is applied to both isolated quaternary photonic crystals and heterostructures. Effective medium theory has been used on photonic crystals and metamaterials in previous works to recover effective parameters, such as refractive index and impedance from transmission and reflection coefficients. We briefly compare analytic and numerical techniques for recovering effective parameters. We then examine how branch point singularities in the real part of the effective refractive index evolve as various parameters in the quaternary crystal, such as tunable layer thickness and loss components of permittivity, change. Construction of the effective refraction index analytically requires knowing the correct branches across the index profile in order to ensure it is both continuous and smooth. Understanding how branch crossings change with other parameters can help prevent abrupt profile changes/discontinuities. 
We then consider the homogenization of a symmetric heterostructures composed of a binary/quaternary/binary, a possible configuration discussed in the second manuscript. The effective refractive index of the system is shown to display brief regions of normal dispersion in the otherwise anomalous regions which correspond to the interfaces states discussed previously. 


\section{ACKNOWLEDGMENTS}

The journey to earn my $\mathrm{PhD}$ here at the University of Rhode Island would not have been possible without the supportive community with whom I have surrounded myself. First, and foremost, I would like to thank Dr. Leonard Kahn for being my doctoral advisor in graduate school. I have known Dr. Kahn for

over 10 years, from the beginning of my undergraduate studies, in 2009. He has been both a fantastic instructor and a great supporting mentor. I am also grateful to the other members of my doctoral committee, Dr. Alexander Meyerovich, Dr. Orlando Merino, and Dr. Mustafa Kulenovic for allowing me this opportunity.

Throughout my undergraduate and graduate studies at URI, I have met many wonderful colleagues with whom I have shared enthusiasm for learning. Sarah Brent, Jennifer Daniels, Aaron Meyer, Adam Card, and many others have provided a great sense of community. The great trio of Steve Pellegrino, Anuradha Weerakkody, and Amer Hodzic have been an amazing group of friends that I am glad I have gotten to know. Our movie and game nights in East Hall have always been a welcome respite from the perils of research. While some friends have had to move away after URI, like Mauricio Escobar and Anton Lobach, I would sometimes receive a humorous text from them that would always lighten my day. I would like to thank Suhail Habeeb, who tragically passed away in 2019, for his company during some late nights in the department as we solved homework problems together or discussed our research. Suhail, you will be greatly missed.

I would also like to give thanks to my amazing family, without whom this incredible journey would not have been possible. My mother, Ann, father, Henry, brother, Brian, and, of course, family cat, Sasha, have given me the strength and determination to pursue this opportunity. 


\section{DEDICATION}

To my grandparents 


\section{PREFACE}

This dissertation has been prepared in manuscript form and consists of three separate academic papers which have either been submitted to journals or intend to be submitted. The first manuscript is an investigation into optical properties of one dimensional dielectric inversion symmetric quaternary photonic crystals. Topology of the band structure for this system is first discussed and compared with that of binary photonic crystals. This is followed by a discussion of the behavior of interface states at the boundary of the heterostructure composed of one binary and one quaternary photonic crystal. This paper is being prepared for submission in OSA Optics Express.

The second paper extends this topic to a heterostructure consisting of an alternationg array of multiple binary and quaternary photonic crystals. The interface states is dependent on whether there is an even or odd number of photonic crystal interfaces in the heterostructure. If there is an odd number, then the properties of the crystals comprising the boundaries will affect the states as well. This paper has been submitted to IOP Journal of Optics.

The third paper examines 1D quaternary photonic crystals and associated heterostructures using effective medium theory. The behavior of branch crossing

points in the effective refractive index is examined as different parameters in the crystal change. Effective medium theory is then applied to a heterostructure consisting of multiple photonic crystals. This paper is being prepared for submission. 


\section{TABLE OF CONTENTS}

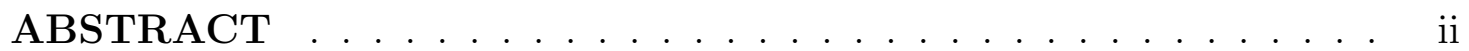

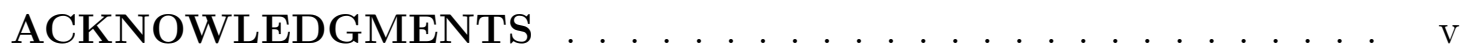

DEDICATION ......................... vi

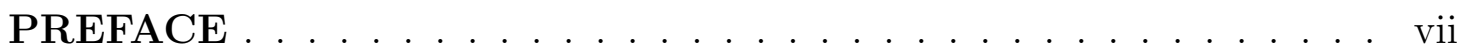

TABLE OF CONTENTS . . . . . . . . . . . . . . v viii

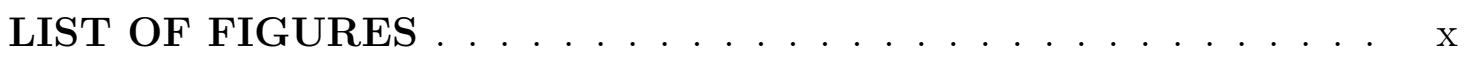

\section{MANUSCRIPT}

1 Topological Photonic States at a 1-D Binary-Quaternary In-

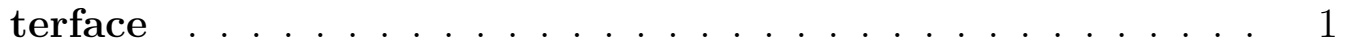

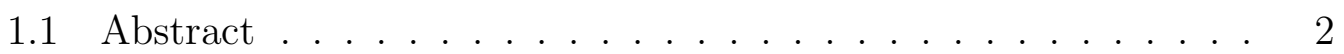

1.2 Introduction . . . . . . . . . . . . . . . . . 2

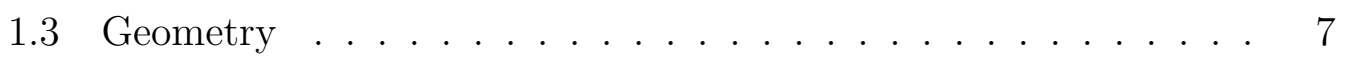

1.4 Results . . . . . . . . . . . . . . . . . . . . 10

1.5 Conclusion . . . . . . . . . . . . . . . . 17

1.6 References . . . . . . . . . . . . . . . . . . . 28

2 Optical States in a 1-D Superlattice with Multiple Photonic Crystal Interfaces . . . . . . . . . . . . . . . . . . 32

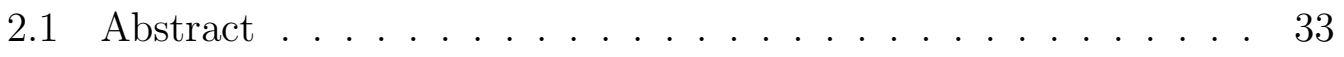

2.2 Introduction $\ldots \ldots \ldots \ldots \ldots \ldots \ldots \ldots \ldots \ldots \ldots$

2.3 Methods . . . . . . . . . . . . . . . . 36

2.4 Results . . . . . . . . . . . . . . . . . . . . . . . . 39 


\section{Page}

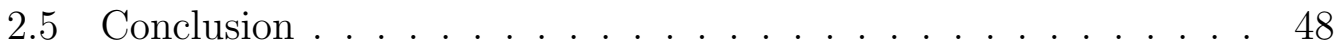

2.6 References . . . . . . . . . . . . . . . . . . . . . 50

3 Branch point crossings and interface states in quaternary photonic crystals using effective medium theory . . . . . . . 53

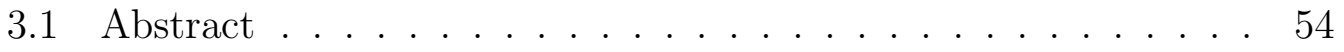

3.2 Introduction . . . . . . . . . . . . . . . . . 54

3.3 Methods . . . . . . . . . . . . . . . . . . . 56

3.4 Results and Discussions . . . . . . . . . . . . . . . 60

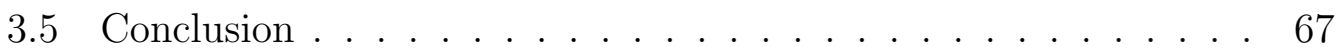

3.6 References . . . . . . . . . . . . . . . . . . . . . . . 69

\section{APPENDIX}

Transfer Matrix Method for general quaternary unit cell _ . . 72 A.1 References . . . . . . . . . . . . . . . . . . 76 


\section{LIST OF FIGURES}

Figure

Page

1 (a) Symmetric unit cells of a binary PC. The primes on $l_{A}^{\prime}$ and $l_{B}^{\prime}$ simply indicate that the lengths of layers $\mathrm{A}$ and $\mathrm{B}$ in the binary $\mathrm{PC}$ are different from the lengths of $\mathrm{A}$ and $\mathrm{B}$ in the quaternary PC. (b) Symmetric unit cells of a quaternary PC. . . . . . . .

2 The interface between two semi-infinite PCs is displayed as a thick black line. On the left hand side (LHS) is a single unit cell from Fig. 1(a). On the RHS (right hand side) is a single unit cell from Fig. 1(b). (a) $d_{C}=0$. Both PCs are identical binaries. (b) As $d_{C}$ increases (while $d_{A}$ and $d_{B}$ change to keep $\gamma$ constant), the PC on the RHS becomes quaternary. (c) Final binary configuration on the RHS when $d_{B} \rightarrow 0$. (d) Final binary configuration on the RHS when $d_{A} \rightarrow 0$. (e) Final configuration on the RHS when both $d_{A} \rightarrow 0$ and $d_{B} \rightarrow 0$. The RHS is a semi-infinite uniform medium. . . . . . . . . . . . . .

3 (a) $\gamma, n_{A}$, and $n_{B}$ are fixed in the system. (b) Surfaces are used to show how the relative thicknesses of layers A (blue), B (red), and C (black) change according to Eqs. (5) and (6). Six different trios of lines of constant $n_{C}$ are highlighted. (c)-(h) Those six trios are plotted sepa rately as profiles with the value of $n_{C}$ displayed above each plot. . . . . . . . . . . . .

4 (a)-(e) The $\left(d_{C}, n_{C}\right)$ parameter space is displayed. The PC can only exist in the shaded areas where $d_{A}, d_{B}, d_{C}$ are all nonnegative. $\gamma$ and $n_{A}$ are constants, with $\gamma<n_{A}$ and $n_{B}$ is free to vary. The dashed line is $n_{C}=\gamma$. The blue and red lines are Eq. (7) and Eq. (8), respectively. In (d), the shaded region vanishes, since the curves overlap. . . . . . . . . . . . 20

5 (a)-(e) Similar to Fig. 4, the $\left(d_{C}, n_{C}\right)$ parameter space is displayed. The PC can only exist in the shaded areas where $d_{A}, d_{B}, d_{C}$ are all non-negative. $\gamma$ and $n_{A}$ are constants, with $\gamma>n_{A}$ and $n_{B}$ is free to vary. The dashed line is $n_{C}=\gamma$. The blue and red lines are Eq. (7) and Eq. (8), respectively. In (b), the shaded region vanishes, since the curves overlap . . . . . . . 
$6 \quad$ System Parameters: $d_{A}=0.2005, \epsilon_{A}=1, \mu_{A}=6, d_{B}=0.5135$, $\epsilon_{B}=1, \mu_{B}=1, d_{C}=0.143, \epsilon_{C}=1, \mu_{C}=3$ (a) $\operatorname{Im}\left(t_{12} \exp \left(i \phi_{A}\right)\right)$ is plotted with respect to frequency, $\xi$. The zeros are displayed as vertical dashed lines. (b) Band structure is plotted. Those three frequencies are superimposed on the plot. The band numbers are displayed in red and the Zak phases in green. There is a very thin $\mathrm{PBG}$ at $\xi \approx 0.9996 . .$. .

$7 \quad$ Band crossing examples for $\phi_{B}=M \phi_{C}$ and $l=1$ (lowest crossing) For each case, $\gamma=1.5, n_{A}=3, n_{B}=1, n_{C}=2$. Red values are band numbers and blue values are Zak phases. Only isolated bands have a Zak phase. (a) $M=\infty$ This is just a binary PC. The first crossing occurs between bands 1 and 2. (b) $M=7$ The first crossing occurs between bands 14 and 15. (c) $M=2.1$ The first crossing occurs between bands 51 and 52. The two inserts show that it can be difficult to distingish a crossing from a thin gap solely by eye. (d) $M=1.59$ The first crossing occurs between bands 417 and $418 \ldots \ldots \ldots \ldots$. . . . . . . . .

8 Locations of the PBG closing points are shown in $\left(\xi, d_{C}\right)$ parameter space. Blue curve are $t_{11}+t_{22}=0$, black curve are $t_{11}+t_{22}=-2$, and red curves are $t_{11}+t_{22}=2$. Green dots are gap closings described by Eq. (23); red dots are closings found graphically or numerically. . . . . . . . . . . . . .

(a) Transmission spectrum about the $3^{\text {rd }} \mathrm{PBG}$ for inversion symmetric quaternary PC with parameters from Fig. 3(a) as well as $\epsilon_{C}=3, \mu_{C}=1$. There is one region where the PBG closes then reopens, at $\left(\xi, d_{C}\right) \approx(0.9996,0.1461)$. Ten unit cells are used. (b) Sign $\left(\zeta^{(3)}\right)$ is displayed as described by Eq. (4). Colors are kept consistent with Ref. [26]. The impedance switches sign after the gaps reopen. (c) Transmission spectrum for combined system of binary and quaternary PCs. For $d_{C}=0$, the system is described by Fig. 2(a). For $d_{C}=d_{C}^{\max }=0.341$, it is described by Fig. 2(d). For $0<d_{C}<d_{C}^{\max }$, the system is described by Fig. 2(b). Five unit cells are used for each PC. Note the topological state after the gap reopens. (d) The state is clearly shown by plotting the implicit equation, $Z_{\text {left }}(\xi)+Z_{\text {right }}\left(\xi, d_{C}\right)=0$, where each term is described by Eq. (16). . . . . . . . . . .

10 (a)-(d) Similar to Fig. 9 except the plots focus on the $10^{\text {th }}$ PBG. There are two regions where the PBG closes then reopens, at $\left(\xi, d_{C}\right) \approx(3.332,0.04508)$ and $\left(\xi, d_{C}\right) \approx(3.334,0.2151) . \ldots$. 
11 (a)-(d) Similar to Fig. 9 except $\epsilon_{C}=1$ and $\mu_{C}=2.25$. PBGs $1-5$ are shown . . . . . . . . . . . . . . 27

12 Schematic of PCs and overall heterostructure. The heterostructure is displayed in the middle as repeating light grey and blue "slabs". Each of these "slabs" represent entire photonic crystals. The grey slabs represent binary PCs, displayed at the bottom. In the bottom diagram, dark green is layer $A$ and dark blue is layer $B$. The vertical dashed lines and double arrow show one unit cell. The primes indicate that the individual layer lengths are different from those of the quaternary PC. Note that the PC is capped on both sides with a half-width of layer $A$, making the unit cells symmetric. The top diagram shows the quaternary $\mathrm{PC}$, which is represented in the middle picture as the light blue regions. In the quaternary $\mathrm{PC}$, layers $A$ and $B$ are the same material as in the binary crystal. Layer $C$ is orange. As in the binary case, the symmetric unit cell is displayed by the double arrow. . . . . . . . . . . . . . .

13 Transmission map for PC heterostructure in $3^{\text {rd }}$ PBG. The stucture consists of alternating binary $(b)$ and quaternary $(q)$ PCs. Each PC has 4 symmetric unit cells. $\epsilon_{A}=6, \epsilon_{C}=3$,

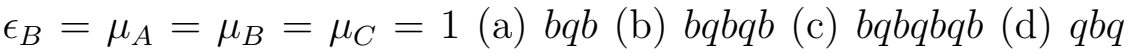
(e) $q b q b q$ (f) $q b q b q b q$ (g) $b q[32]$ (h) $b q b q$ (i) $b q b q b q \ldots$. . . . . .

14 (a) Transmission resonance behavior for PC heterostucture $b q \ldots q b$ as the number of central binary crystals varies. Each $b$ and $q$ represents 4 unit cells ( $b / 2$ is 2 unit cells). (b) Central region of $(\mathrm{a}) . \ldots \ldots \ldots \ldots$

15 Real and imaginary parts of $\epsilon_{C}$ for metallic layer $C$. Plasma frequency $\xi_{p}=2$ and collision frequency $g=10^{-10}$. Frequencies are scaled according to $\xi=f \Lambda / c_{0}$. . . . . . . . . . . . .

16 (a) Transmission map behavior for PC heterostucture $b q$ where the quaternary PC contain metallic layer $C . \epsilon_{A}=6, \epsilon_{B}=\mu_{A}=$ $\mu_{B}=\mu_{C}=1$. $\epsilon_{C}$ is given by Eq. 36. Note the five topological states indicated by the arrows. The color scheme is logarithmic. (b) These states correspond to where the imaginary part of the impedance is zero. . . . . . . . . . . . . . . . . 46

17 Transmission map for a double interface heterostructure. Layer $C$ of the quaternary $\mathrm{PC}$ is metallic. Configuration is (a) $q b q(\mathrm{~b}) b q b 47$ 
18 Transmission cross section for the heterostucture in Fig. 12 with layer $C$ having frequency dependence described by Eq. 36. All other $\epsilon$ and $\mu$ values are the same as in Fig. 13. (a) Transmission for structures of form $q b \ldots b q$. (b) Zoomed in version of left collection of peaks in (a). (c) Transmission for structures of form $b q \ldots q b . \ldots \ldots \ldots$. . . . . . . . . . . . . . . 4

19 Analogous coupled oscillator model of the heterostructure shown in Fig 12. The spring constants represent the PCs and the masses represent interfaces between PCs. . . . . . . . . . . .

20 Schematic showing the splitting of the original interface state. The number of interfaces is $N$. The left diagram represents an even number of interfaces while the right one is for an odd number of interfaces. . . . . . . . . . . . . . 48

21 Inversion symmetric quaternary photonic crystal . . . . . . . 56

22 Example of effective parameters of 1 unit cell from Fig. 21a. In the original unit cell $d_{A}=0.14207, d_{B}=0.45793, d_{C}=0.2$, $\epsilon_{A}=6+0.2 i, \mu_{A}=1, \epsilon_{B}=1, \mu_{B}=1, \epsilon_{C}=3+0.2 i$, and $\mu_{C}=1$.(a) Real and imaginary effective impedance. (b) Real component of refractive index. The values of $m$ specify the branches to ensure $n_{R}$ is continuous and smooth. The black dots highlight the points where the branches connect. (c) Real component of refractive index after nonphysical solutions are removed. (d) Imaginary component of refractive index. . . . . . 59

23 Difference in transmission calculated analytically with Eqs. 46 and 47 and numerically with Eqs. 48 and 49 (a) 1 unit cell (b) 3 unit cells. Note the regions given by the arrows where convergence is poor. With 3 unit cells the band gaps are more visible, showing better agreement than surrounding areas. . . . 61

24 Behavior of (a) $1^{\text {st }}(\mathrm{b}) 2^{\text {nd }}$ (c) $3^{\text {rd }}$ crossing point as $\operatorname{Im}\left(\epsilon_{A}\right)$ changes 62

25 Behavior of (a) $1^{\text {st }}$ (b) $2^{\text {nd }}$ (c) $3^{\text {rd }}$ crossing point as $\operatorname{Im}\left(\epsilon_{C}\right)$ changes 63

26 Behavior of (a) $1^{\text {st }}$ (b) $2^{\text {nd }}$ (c) $3^{\text {rd }}$ crossing point as $d_{C}$ changes .64 
27 (a) Two transmission peaks in $3^{\text {rd }}$ band gap for heterostructure: binary/quaternary/binary. Parameters are: $\gamma=1.5, \epsilon_{A}=6+$ $0.01 i, \mu_{A}=1, \epsilon_{B}=1, \mu_{B}=1, \epsilon_{C}=3+0.01 i, \mu_{C}=1, d_{C}=0.25$. $d_{A}$ and $d_{B}$ are given by Eqs. $40 \& 41$. (b) Real part of effective refractive index. The boxed region is in (c). (c) The arrows indicate the regions that correspond to the transmission peaks. From left to right, the physcial solution smoothly transitions across the following branches: purple $\rightarrow$ green $\rightarrow$ cyan $\rightarrow$ yellow. 66

A.1 Diagram of the PC used in the calculations below . . . . . . . 72 


\title{
MANUSCRIPT 1 \\ Topological Photonic States at a 1-D Binary-Quaternary Interface
}

\author{
Nicholas J Bianchi and Leonard M Kahn \\ University of Rhode Island \\ Physics Department
}

This manuscript is being prepared for submission in OSA Optics Express. 


\section{$1.1 \quad$ Abstract}

The existence of topological interface states is investigated in a heterostructure consisting of a binary photonic crystal and a quaternary photonic crystal. The individual crystals possess inversion symmetric unit cells. In this work, a photonic crystal is made quaternary by inserting a tunable third layer into two different locations in the unit cell. Conditions are established that describe where the quaternary crystal can exist in parameter space subject to constraints. The closing of band gaps is discussed for different optical path ratios. When the binary and quaternary crystals share an interface, optical states appear at the interface when the two crystals have different signs for the surface impedance. The evolution of the states is displayed as the geometry of the quaternary crystal changes.

\subsection{Introduction}

A photonic crystal (PC) is an periodic array of dielectrics and/or conductors able to scatter electromagnetic (EM) fields, where the scattering elements and incident wavelengths are of similar size [1]. This periodicity implies that a PC possesses discrete translational symmetry. Therefore, as in solid state lattices, EM waves are described using Bloch's Theorem [2], and the wave equation can be solved for the modes allowed in the PC. Destructive interference due to multiple scattering inside the PC produces frequency ranges in which no mode is allowed to propagate through the crystal regardless of crystal momentum (i.e. Bloch wavevector). These regions of reduced transmission are known as photonic band gaps (PBG), although in 1-D systems they are also called stop bands. While investigations about 1-D photonic systems began with Lord Rayleigh [3], PBG research in two and three dimensions did not accelerate until the works of Yablonovitch [4, 5] and John [6] about a century later. Applications of PBGs in PCs are numerous, including

dielectric mirrors [1], channeling EM modes through photonic slab waveguides [7] 
and optical fibers [8], and construction of defect states [9, 10]. PBGs are also not just limited to PCs; photonic aperiodic structures $[11,12,13,14]$ and certain types of disordered hyperuniform [15] media can also support band gaps, due to isotropy $[16,17,18]$.

When multiple PCs are joined together, a superlattice can be constructed that can possess optical properties not observed in an isolated crystal. Among these properties is the ability to localize EM fields at an interface. This was theoretically demonstrated by Kavokin et al. [19], using two adjacent lossless PCs with different periods. The interface states that developed at the boundary of the individual crystals were called optical Tamm states (OTS), due to similarities with electronic surface modes discovered by Tamm [20], and were found to be strongly dependent on the order of the individual layers in the crystals. Follow up investigations by Vinogradov et al. (2006) [21] demonstrated that these OTSs require the normal wavevector to decrease with distance from the interface on both sides. Since there is a PC on both sides, the only way this can happen is if the wave is trying to propagate in a PBG. This implies that a necessary condition for the formation of interface modes is that PBGs of the individual PCs in the superlattice must overlap. These states were experimentally verified soon after and appear as a sharp peak in transmission spectra [22]. Kang et al. [23] used a Bloch Wave Expansion technique for symmetric and asymmetric unit cells to show that OTSs would appear if impedance matching was satisfied at the interface and confirmed the idea that the order of layers in a unit cell mattered for the appearance of states [19]; however,no physical connection between the appearance of states and the layer order was found [23]. While OTSs were mostly studied in asymmetric unit cell configurations, Vinogradov et al. (2010) [24] showed that symmetric unit cells were also valid. Interface states with symmetric unit cells 
were classified as optical Shockley [25] states (OSSs); however, it was determined that the underlying physical mechanism that produced OTSs and OSSs was the same, thus all optical states are referred to as Tamm states, although many papers simply call them interface states. While the bulk band structure for an infinite crystal will be unaffected by the symmetry of the unit cell, the exact location of the interface state will shift slightly [24].

Utilizing a symmetric unit cell, a special type of OTS called a topological interface state can be studied. As stated before, the existence of interface states in a superlattice is strongly dependent on the order of the layers comprising the PCs. Xiao et al. [26] was able to explain the surface impedance of a PC in terms of a topological invariant known as the Zak phase [27]. A Zak phase is assigned to each isolated bulk band in the band structure. It was shown that interface states emerge in the band gaps if these phases change value via a topological phase transition. One way these phase transitions can occur is if the order of layers in unit cells on one side of the interface is reversed while on the other side it is not. The electric field will acquire a Zak phase (for each band) as the Bloch wavevector, $\kappa$, travels on a closed path around the $1^{\text {st }}$ Brillouin zone. Since the system is $1 \mathrm{D}$, this path is a ring. If the $\mathrm{PC}$ is $D$-dimensional, the trajectory that $\kappa$ traces in momentum space would exist on the surface of a $D$-torus. For a PC whose unit cell possesses inversion symmetry, the Zak phase is a convenient measure of topological phase of the band structure as it is constrained to 0 or $\pi$, depending on the inversion center [27]. For EM systems with period $\Lambda$, the Zak phase can be written as [26]:

$$
\theta_{n}^{\mathrm{zak}}=\int_{-\pi / \Lambda}^{\pi / \Lambda} i\left\langle u_{n, \kappa}\left|\epsilon \partial_{\kappa}\right| u_{n, \kappa}\right\rangle d \kappa
$$

where

$$
i\left\langle u_{n, \kappa}\left|\epsilon \partial_{\kappa}\right| u_{n, \kappa}\right\rangle=i \int_{\text {unit cell }} u_{n, \kappa}^{*}(z) \epsilon(z) \partial_{\kappa} u_{n, \kappa}(z) d z
$$


represents the Berry connection. In Eq. (2), $\epsilon(z)$ is the relative permittivity across the unit cell, and $u_{n, \kappa}(z)$ is the periodic function from Bloch's Theorem, $E_{n, \kappa}(z)=\exp (i \kappa z) u_{n, \kappa}(z)$, where $E_{n, \kappa}(z)$ is the electric field. The label $n$ specifies the isolated band.

A topological interface state will appear if the surface impedances of the PCs on both sides of the interface sum to zero [26]:

$$
Z_{\text {left }}+Z_{\text {right }}=0
$$

Since the impedance in a PBG is imaginary, it can be written as $Z / Z_{0}=i \zeta$, where:

$$
\operatorname{sign}\left(\zeta^{(n)}\right)=(-1)^{n+l} \exp \left(i \sum_{m=0}^{n-1} \theta_{n}^{\mathrm{zak}}\right)
$$

with $Z_{0}$ being the vacuum impedance. The variable $l$ indicates the number of band crossings (Dirac points) in the band structure below gap $n$. In a binary PC, a Dirac point will occur if the ratio of the optical path lengths of the two layers is a rational number $[26,28]$.

After Xiao's paper, research in this field began to rapidly expand. Experimental measurements of Zak phase in a 1-D $\mathrm{SiO}_{2}-\mathrm{TiO}_{2}$ composite structure were conducted by first measuring reflection phase [29, 30]. Other experiments have measured Zak phases through direct observation of interface states [31]. Recent theoretical work has shown that by manipulating the unit cell inversion centers, a superlattice can be designed that supports topological states in every PBG[32]. The robustness of topological states in photonic systems with a finite number of layers has also been examined as the unit cell number varies [33]. Some photonic systems simultaneously support topological and Fano resonances [34]. Other works have extended these ideas to include PCs including metallic layers [35, 36, 37].

While the concept of interface states at the boundary of two inversion symmetric binary PCs is well understood, the literature is sparse about what happens 
when an additional layer is added to one of the crystals $[38,39,40]$. More specifically, we consider inserting an additional layer inbetween every original layer of the binary PC. The binary crystal is displayed in Fig. 1(a) while the new crystal is shown in Fig. 1(b). Despite being composed of three different materials, this new $\mathrm{PC}$ is not ternary. In order to keep the unit cell inversion symmetric, two layer $C$ 's must be included, thus giving it four layers and being referred to as a quaternary PC. The primes are used to indicate that the thicknesses of layers $A$ and $B$ can be different between the two PCs, even if the respective layers are composed of the same material.

Section III discusses the geometry of the PCs used in this work. With a constant optical path length, as a new layer $(C)$ is introduced to a binary PC, the original layers will grow or shrink, depending on their respective refractive indices. If this new layer is large enough, the now quaternary $\mathrm{PC}$ will become binary again, but with a different periodic configuration. A parameter space with dimensions of the refractive index and thickness of the new layer is also constructed. As properties of the PC are altered, the acceptable region within this parameter space where the PC can exist will change. Section IV discusses topological phase and band crossing conditions for a quaternary PC and contrasts them with the more familar binary PC. Transfer matrix elements are derived for a quaternary PC and crossing conditions are written in terms of a ratio between two propagation phase terms. As this ratio becomes irrational, the first band crossing occurs at higher and higher bands. Next, several examples are given of interface state behavior in a binary-quaternary structure as the geometry changes. It is shown, especially at higher frequencies, that when an additional layer is introduced to make half of the structure quaternary, an interface state can appear but then disappear again before the quaternary PC becomes another different binary crystal. Section V concludes 
this work.

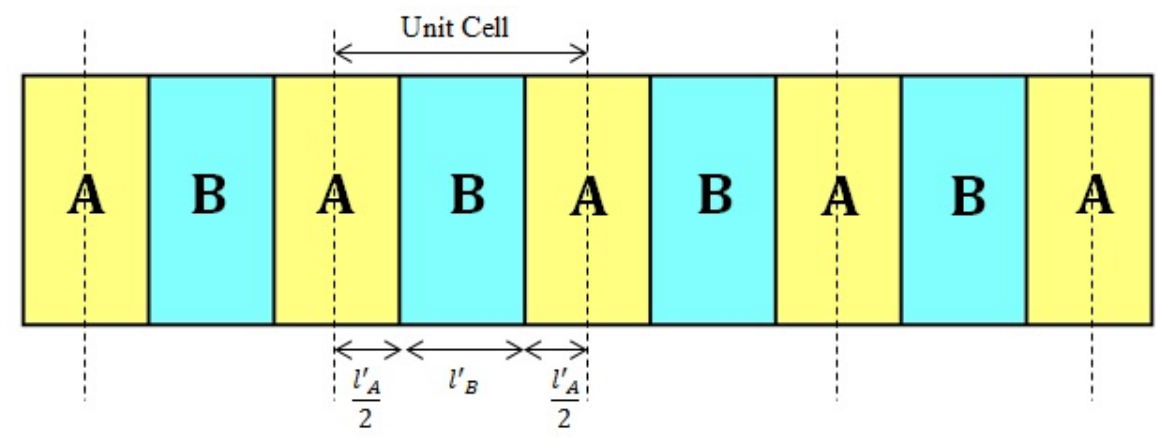

(a)

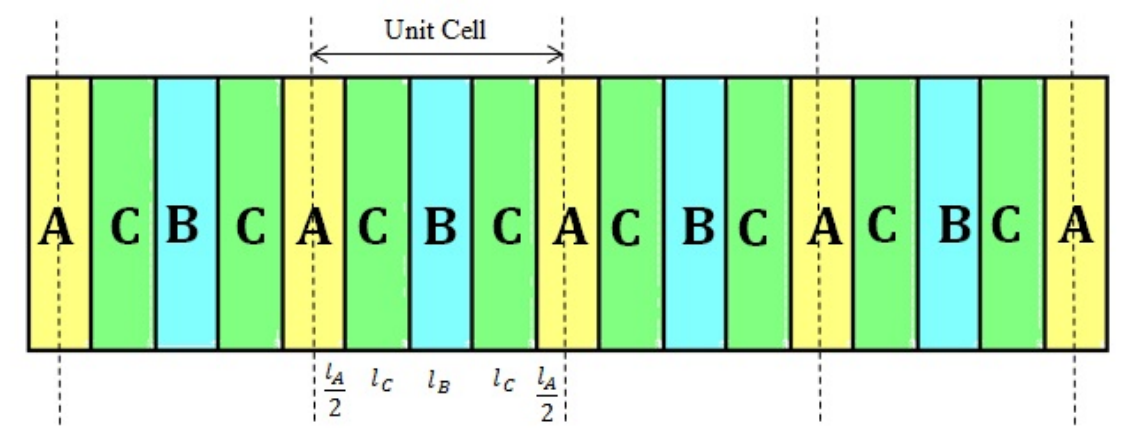

(b)

Figure 1: (a) Symmetric unit cells of a binary PC. The primes on $l_{A}^{\prime}$ and $l_{B}^{\prime}$ simply indicate that the lengths of layers $\mathrm{A}$ and $\mathrm{B}$ in the binary $\mathrm{PC}$ are different from the lengths of A and B in the quaternary PC. (b) Symmetric unit cells of a quaternary PC.

\subsection{Geometry}

Before discussing the results of this investigation, it is benifical to establish some dimensionless quantities that will make scaling more natural. Since any periodic PCs are constructed of identical unit cells, we need only consider a single unit cell. The period and optical path length of the unit cell are $\Lambda=l_{A}+l_{B}+2 l_{C}$ and $\Gamma=n_{A} l_{A}+n_{B} l_{B}+2 n_{C} l_{C}$, respectively. The individual layers have thicknesses $l_{i}$ and refractive indices $n_{i}$, for $i=\{A, B, C\}$. In this paper, $\Lambda$ and $\Gamma$ are held constant. Therefore, we define a parameter, $\gamma \equiv \Gamma / \Lambda \geq 1$. The layer thicknesses are made dimensionless with $d_{i} \equiv l_{i} / \Lambda$.With this information: 


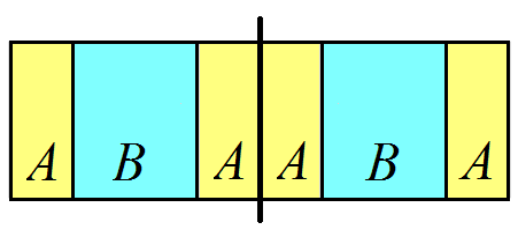

(a)

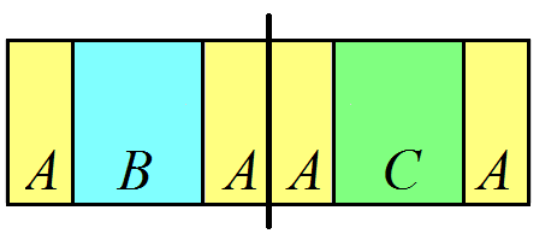

(c)

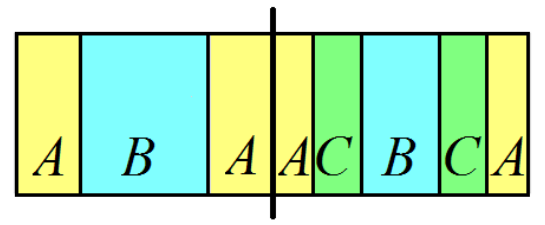

(b)

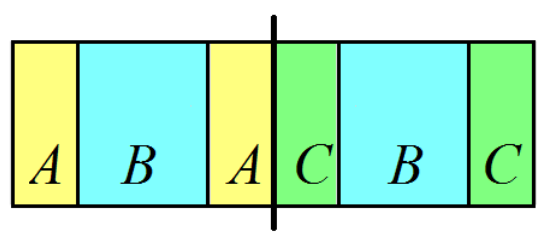

(d)

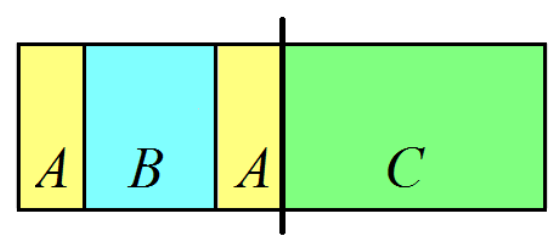

(e)

Figure 2: The interface between two semi-infinite PCs is displayed as a thick black line. On the left hand side (LHS) is a single unit cell from Fig. 1(a). On the RHS (right hand side) is a single unit cell from Fig. 1(b). (a) $d_{C}=0$. Both PCs are identical binaries. (b) As $d_{C}$ increases (while $d_{A}$ and $d_{B}$ change to keep $\gamma$ constant), the PC on the RHS becomes quaternary. (c) Final binary configuration on the RHS when $d_{B} \rightarrow 0$. (d) Final binary configuration on the RHS when $d_{A} \rightarrow 0$. (e) Final configuration on the RHS when both $d_{A} \rightarrow 0$ and $d_{B} \rightarrow 0$. The RHS is a semi-infinite uniform medium.

$$
\begin{aligned}
& d_{A}=\frac{\gamma-n_{B}-2\left(n_{C}-n_{B}\right) d_{C}}{n_{A}-n_{B}} \\
& d_{B}=\frac{\gamma-n_{A}-2\left(n_{C}-n_{A}\right) d_{C}}{n_{B}-n_{A}}
\end{aligned}
$$

In Eqs. (5) and (6), $d_{C}$ is an independent variable. As $d_{C}$ changes, $d_{A}$ and $d_{B}$ increase or decrease depending on the relative sizes of $\gamma, n_{A}$, and $n_{B}$. An example of this behavior is shown in Fig. 3. For the given parameters in Fig. 3(a), $d_{A}, d_{B}$, and $d_{C}$ can exist only on the blue, red, and black surfaces, respectively, shown in 
Fig. 3(b). The bright lines in the surfaces represent behavior of $d_{i}$ vs $d_{C}$ for six different values of $n_{C}$. That is, each $n_{C}$ value presents a particular vertical slice of Fig. 3(b), which is shown in Figs. 3(c) - 3(h). While each of the six profiles show

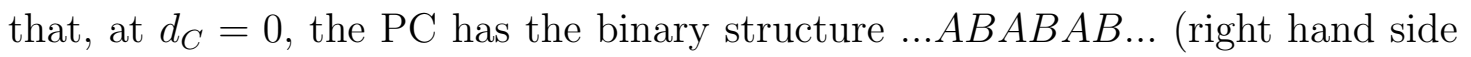
(RHS) of Fig. 2(a)), by altering $n_{C}, d_{A}$ and $d_{B}$ can display different rates of change with respect to $d_{C}$. In Figs. $3(\mathrm{c})$ and $3(\mathrm{~d}), n_{C}<\gamma$, so as $d_{C}$ increases, $d_{B}$ reaches 0 before $d_{A}$. When this happens, the PC configuration becomes ...ACACAC... (RHS of Fig. 2(c)). Note that in Fig. 3(c), since $n_{B}=n_{C}, d_{A}$ is flat ${ }^{1}$. Also, as $n_{C} \rightarrow \gamma$, Eqs. (5) and (6) can stay positive for larger values of $d_{C}$. This continues until $n_{C}=\gamma$, in Fig. 3(e). At this point, a cusp is reached and $d_{A}=d_{B}=0$ when $d_{C}=0.5$. Therefore, the PC become a uniform dielectric, ...CCCCCC... (RHS of Fig. 2(e)). As $n_{C}$ increases further, in Fig. 3(f) to $\sqrt{3}, d_{A}$ drops to 0 first. When this occurs, the PC takes the form ...CBCBCB... (RHS of Fig. 2(d)). The maximum $d_{C}$ value also starts to decrease. In Fig. $3(\mathrm{~g}), n_{C}$ increases until $n_{C}=n_{A}$, at which point $d_{B}$ become flat. Finally, $d_{B}$ has a positive slope in Fig. 3(h), as $n_{C}>n_{A}$.

It is important to reiterate that in the example described in Fig. $3, n_{A}$ and $n_{B}$ are held constant while $n_{C}$ is free to vary. The surface boundaries in Fig. 3(b) are shaped by the conditions that the layer thicknesses must be non-negative and their collective sum must be the period (i.e $d_{A}+d_{B}+2 d_{C}=1$ ). In other words, we can imagine viewing Fig. 3(b) from a "top-down" perspective, projecting the surfaces into a 2-D parmeter space of coordinates $\left(d_{C}, n_{C}\right)$ In this space, setting Eqs. (5) and (6) to 0 and solving each for $n_{C}$ yields:

$$
\left.n_{C}\right|_{d_{A}=0}=\frac{\gamma-n_{B}}{2 d_{C}}+n_{B}
$$

\footnotetext{
${ }^{1}$ While $n_{B}=1$ in this example for simplicity, if $1<n_{B}<\gamma$, then $d_{A}$ would have a positive slope for $n_{C}<n_{B}$. The slope would go to 0 as $n_{C} \rightarrow n_{B}$
} 


$$
\left.n_{C}\right|_{d_{B}=0}=\frac{\gamma-n_{A}}{2 d_{C}}+n_{A}
$$

Eqs. (7) and (8) are displayed in Fig. 4 and represent the boundaries in parameter space. The blue curve is Eq. (7) and the red curve is Eq. (8). The shaded area is the region where the PC is allowed to exist. Here $\gamma<n_{A}$ and both constants are given the same values they had in Fig. 3(a); however, now $n_{B}$ is allowed to change. In Fig. 4(a), since $n_{B}<\gamma$, the shaded region is unbounded as $n_{C} \rightarrow \infty$, although Eq. (7) asymptotically approaches $d_{C}=0$. Fig. 4(a) represents the parameter space for Fig. 3(b). As $n_{B} \rightarrow \gamma$, the shaded area transforms into Fig. 4(b). Now there is a strict upper bound on $n_{C}$, above which the PC cannot exist. Further increasing $n_{B}$ causes Eq. (7) to become concave-down. In Fig. 4(c), this cuts off $d_{C}=0$ from the available region. This means that in order for the PC to exist, it must contain layer $C$ everywhere. As $n_{B} \rightarrow n_{A}$, in Fig. $4(\mathrm{~d})$, the two curves overlap, causing the region to vanish. It then opens again in Fig. 4(e), but with Eq. (7) ahead of Eq. (8) ${ }^{2}$.

Fig. 5 displays a similar situation to Fig. 4 except now $n_{A}<\gamma$. The main difference is that $n_{C}=\gamma$ serves as a lower bound rather than an upper bound.

\subsection{Results}

Now that the conditions for the existence of an inversion-symmetric 4-layer $\mathrm{PC}$ and its connection to the binary crystal have been shown, it is easier to discuss the conditions in which a topological state can form. By using the transfer matrix method (See Appendix), we can extend the familiar transfer matrix elements from a binary unit cell $[26,41]$ :

\footnotetext{
${ }^{2}$ If $\gamma$ and $n_{B}$ were constants, with $\gamma<n_{B}$ and $n_{A}$ allowed is change, then the positions of the red and blue curves would be reversed in each plot of Fig. 4.
} 


$$
\begin{gathered}
t_{11}=e^{i \phi_{A}}\left(\cos \phi_{B}+i z_{A B}^{+} \sin \phi_{B}\right) \\
t_{12}=i e^{-i \phi_{A}} z_{A B}^{-} \sin \phi_{B}
\end{gathered}
$$

to a symmetric 4 layer unit cell:

$$
\begin{gathered}
t_{11}=e^{i \phi_{A}}\left[\cos \phi_{B} \cos \left(2 \phi_{C}\right)+\left(i z_{A C}^{+} \cos \phi_{B}-z_{B C}^{+} \sin \phi_{B}\right) \sin \left(2 \phi_{C}\right)\right. \\
\left.+i\left(z_{A B}^{+} \cos ^{2} \phi_{C}-z_{A B C}^{+} \sin ^{2} \phi_{C}\right) \sin \phi_{B}\right] \\
t_{12}=i e^{-i \phi_{A}}\left[z_{A C}^{-} \cos \phi_{B} \sin \left(2 \phi_{C}\right)+\left(z_{A B}^{-} \cos ^{2} \phi_{C}-z_{A B C}^{-} \sin ^{2} \phi_{C}\right) \sin \phi_{B}\right]
\end{gathered}
$$

where $\phi_{i} \equiv k_{i} l_{i}=2 \pi n_{i} d_{i} \xi$ for dimensionless frequency $\xi=f \Lambda / c_{0} . \quad f$ is the frequency and $c_{0}$ is the speed of light in vacuum. The impedance mismatch terms are defined as:

$$
\begin{gathered}
z_{i j}^{ \pm} \equiv \frac{1}{2}\left(\frac{z_{i}}{z_{j}} \pm \frac{z_{j}}{z_{i}}\right) \\
z_{A B C}^{ \pm} \equiv \frac{1}{2}\left(\frac{z_{A} z_{B}}{z_{C}^{2}} \pm \frac{z_{C}^{2}}{z_{A} z_{B}}\right)
\end{gathered}
$$

for relative impedance, $z_{i}$. It is easy to show that Eq. (11) reduces to Eq. (9) and Eq. (12) reduces to Eq. (10) when either $d_{B} \rightarrow 0$ or $d_{C} \rightarrow 0$. A third case, $d_{A} \rightarrow 0$, will also work, but is a bit more subtle; in order for this case to simplify, the substitution $z_{A} \rightarrow z_{C}$ must be made. Adding Eq. (11) with its complex conjugate yields the dispersion relation (See Appendix):

$$
\begin{aligned}
\cos (\kappa \Lambda) & =\cos \phi_{A} \cos \phi_{B} \cos \left(2 \phi_{C}\right) \\
& -\left(z_{B C}^{+} \cos \phi_{A} \sin \phi_{B}+z_{A C}^{+} \sin \phi_{A} \cos \phi_{B}\right) \sin \left(2 \phi_{C}\right) \\
& +\left(z_{A B C}^{+} \sin ^{2} \phi_{C}-z_{A B}^{+} \cos ^{2} \phi_{C}\right) \sin \phi_{A} \sin \phi_{B}
\end{aligned}
$$


With Eqs. (11) and (12), we can use the expression for surface impedance in Ref. [26],

$$
\operatorname{Im} \frac{Z}{Z_{0}}=z_{A} \frac{t_{12} \exp \left(i \phi_{A}\right)+\left(\exp (i \kappa \Lambda)-t_{11}\right)}{t_{12} \exp \left(i \phi_{A}\right)-\left(\exp (i \kappa \Lambda)-t_{11}\right)}
$$

Note that Eq. (16) is simply the ratio of Eqs. (A.1) and (A.2) with eigenvector components: $a_{0}^{(1)}=t_{12}$ and $b_{0}^{(1)}=\exp (i \kappa \Lambda)-t_{11}$ [41]. Inserting Eq. (16) into Eq. (3), gives a complete equation for a topological interface state. By letting $\kappa \Lambda=n \pi+i x$ [41], the Bloch phase in Eq. (16) can be re-written in terms of the dispersion relation:

$$
e^{i \kappa \Lambda}=(-1)^{n} e^{-x}
$$

where,

$$
\cosh (x)=(-1)^{-n} \cos (\kappa \Lambda)
$$

The value $n$ denotes the band gap number and $x$ represents a decay factor.

As in Ref. [26], calculating the Zak phase for each isolated band, from Eq. (15), requires finding the set of frequencies, $\xi$, in which $\operatorname{Im}\left(t_{12} \exp \left(i \phi_{A}\right)\right)=0$, assuming the center of layer $A$ is chosen as the center of inversion. If such a value of $\xi$ intersects a band $n>0$, then for that band, $\theta_{n}^{\text {zak }}=\pi$; for all bands not intersected, $\theta_{n}^{\text {zak }}=0$. For the binary PC, the $\xi$ and thus the Zak phases can be found analytically for all bands. This is done using Eq. (10) [26]: $z_{A B}^{-} \sin \phi_{B}=0$. For the $0^{\text {th }}$ band:

$$
\exp \left(i \theta_{0}^{\mathrm{zak}}\right)=\operatorname{sign}\left(z_{A B}^{-}\right)
$$

For all other bands, $\sin \phi_{B}=0$. A similar procedure can be done for the 4-layer unit cell, using Eq. (12); however, one quickly realizes that now $\xi$ cannot be found analytically. Furthermore, the situation is complicated by the fact that $\theta_{0}^{\text {zak }}$ cannot be separately calculated. While it is still true that $\theta_{n>0}^{\text {zak }}=\pi$ for bands intersected by $\xi$, this rule does not appear to consistently hold for the $0^{\text {th }}$ band. In addition, for bands $n>0$, there may be instances where two different $\xi$ values intersect the 
same band. If this happens, that band has $\theta_{n>0}^{\mathrm{zak}}=0$; two crossings are treated as no crossing. An example of this behavior is displayed in Fig. 6. Note that in Fig. 6(a), the local maximum would be equal to zero for $d_{C} \approx 0.1425821$. In that case, the blue and magenta $\xi$ values would become repeated solutions, and despite there only being one root, $\theta_{3}^{\text {Zak }}$ would still be 0 since an infintesimal increase or decrease in $d_{C}$ would result in a double crossing or no crossing, respectively. The dashed lines in Fig. 6(a) are plotted with the band structure in Fig. 6(b). As $d_{C}$ increases, the blue $\xi$ will shift down. When $d_{C} \approx 0.1461$, bands 2 and 3 will cross, closing the gap. At that moment, the blue line will exist exactly where the bands cross, at $\xi \approx 0.9996$. Increasing $d_{C}$ further reopens the gap, but now the blue line has moved down to the $2^{\text {nd }}$ band. The $2^{\text {nd }}$ band would now have a double crossing with $\theta_{2}^{\text {zak }}=0$ and the $3^{\text {rd }}$ band would now have a single crossing with $\theta_{3}^{\text {zak }}=\pi$.

Analytic results for band crossings can be obtained if the constraint, $M \phi_{C}=$ $\phi_{B}$, is applied, for $M \in \mathbb{Q}$, assuming that all layer widths remain non-negative. Applying this condition to Eqs. (5) and (6) yields:

$$
\begin{aligned}
& d_{A}=\frac{(M+2) n_{B} n_{C}-\gamma\left(2 n_{B}+M n_{C}\right)}{(M+2) n_{B} n_{C}-n_{A}\left(2 n_{B}+M n_{C}\right)} \\
& d_{B}=\frac{M n_{C}\left(\gamma-n_{A}\right)}{(M+2) n_{B} n_{C}-n_{A}\left(2 n_{B}+M n_{C}\right)} \\
& d_{C}=\frac{n_{B}\left(\gamma-n_{A}\right)}{(M+2) n_{B} n_{C}-n_{A}\left(2 n_{B}+M n_{C}\right)}
\end{aligned}
$$

It is easy to check that as $M \rightarrow \infty, d_{C} \rightarrow 0$ while $d_{A}$ and $d_{B}$ reduce to their respective binary expressions. This constraint allows for the following band crossing condition to hold $[39,40]^{3}$ :

$$
n_{A} d_{A}: n_{B} d_{B}: 2 n_{C} d_{C}=m_{1}: m_{2}: m_{3}
$$

\footnotetext{
${ }^{3}$ The factor of 2 in the last term is due to the total length of layer $C$ being $2 d_{C}$. In $[39,40]$, there is only a single layer $C$, hence no extra factor
} 
for $\left\{m_{1}, m_{2}, m_{3}\right\} \in \mathbb{N}$. Therefore, bands $l\left(m_{1}+m_{2}+m_{3}\right)$ and $l\left(m_{1}+m_{2}+m_{3}\right)-1$ will cross at frequency $\xi_{\text {cross }}=l\left(m_{1}+m_{2}+m_{3}\right) /(2 \gamma)$, where $l \in \mathbb{N}^{+}$. It is productive to illustrate these crossing with examples. In Fig. 7, four examples of band crossings are shown, each with a different $M$ value. To ensure that a crossing exists, all refractive indices are rational numbers. Therefore, Eq. (23) can be written as a trio of non-negative integers. In Fig 7(a), when one of the terms is 0 , the PC becomes binary and the first crossing occurs at a low frequency. In fact, for the particular refractive index values used in this example, only the $0^{\text {th }}$ band is isolated. Fig 7(b)- 7(d), show that as the number of non-repeating digits in $M$ increases, the first crossing occurs at ever higher band numbers, tending to $\infty$ as $M$ becomes irrational. Note that in Fig 7(b), the lower band in the crossing pair is even, leading to the crossing happening at the band edge; in the other three plots, the lower band in the crossing pair is odd, so the crossing occurs at the band center. Fig 7(c) also highlights the difficulty in trying to identify band crossing visually. The larger graph appears to show a crossing at $\xi=18$; however, this is simply due to a lack of resolution. The left insert shows that there is a very narrow gap at this frequency value, while the right insert does indeed show a Dirac point at $\xi=52 / 3$. If $M$ were to slightly increase from $2.1 \rightarrow 2.2$, then bands 51 and 52 would separate while bands 53 and 54 cross, for $l=2$ and $\left\{m_{1}, m_{2}, m_{3}\right\}=\{6,11,10\}$. Note that while Eq. (23) is sufficent to allow band crossings, it is not necessary. This ratio can only predict values of $\xi_{\text {cross }}$ that are integer multiples of $(2 \gamma)^{-1}$.

The distinction between the crossing points described by Eq. (23) and those that are not is displayed in Fig. 8. The points governed by the ratio are green and occur at integer multiples of $(2 \gamma)^{-1}$ (1/3 for these examples). Since the refractive indices in both Fig 8(a) and 8(b) are the same, these three points remain the same. Note that Fig. $7(\mathrm{~b})$ is the band structure for Fig. $8(\mathrm{a})$ when $d_{C}=0.05(M=7)$. 
If the band diagram was based on the parameters from Fig. 8(b), it would look slightly different, but the crossing point at $\xi=5$ would remain unchanged. The crossing points denoted in red are not described by Eq. (23). When $\epsilon_{A}$ and $\mu_{A}$ switch values, these points change position, implying that they depend on the impedance.

Another interesting difference between binary and inversion symmetric quaternary PCs is the closing of the first band gap. For a binary crystal, the first PBG will only close when there is impedance matching across the layers of the unit cell (i.e. $z_{A}=z_{B}$ ); however, this matching would cause either $d_{A}$ or $d_{B}$ (see Eq. (5) or Eq. (6)) to be negative. Even if layers $A$ and $B$ are forced to have positive widths (by ignoring the constraints of $\Lambda$ and $\Gamma$ ), every band gap would close, since the PC would exhibit perfect transmission for all frequencies. For the quaternary PC with layer width dictated by Eq. (5) or Eq. (6), the first gap is allowed to close even if the others remain open. Despite $z_{A} \neq z_{B} \neq z_{C}, d_{C}$ can be tuned so that the lowest gap closes. This closing is not associated with Eq. (23), since two of the $m$ 's are zero, and thus the associated Dirac point in $\left(\xi, d_{C}\right)$ space must be found numerically.

By setting this quaternary PC adjacent to a binary PC, interesting topological behavior is observed. For both PCs, let $\gamma=1.5, \epsilon_{A}=6, \mu_{A}=1, \epsilon_{B}=1, \mu_{B}=1$ (See Fig. 3). Three examples of topological state behavior are shown in Fig. 9, Fig. 10, and Fig. 11. For the quaternary PC let the parameters for layer $\mathrm{C}$ be $\epsilon_{C}=3, \mu_{C}=1$ for Fig. 9 and Fig. 10 , and $\epsilon_{C}=1, \mu_{C}=2.25$ for Fig. 11 . Let us first consider Fig. 9. A transmission map about the $3^{\text {rd }} \mathrm{PBG}$ of the isolated quaternary PC is displayed in Fig. 9(a), showing two transmission deserts. At $d_{C} \approx 0.1461$, a Dirac point occurs. In Fig. $9(\mathrm{~b})$, it is shown that this crossing produces a change in the sign of the surface impedance of the gap, thus producing a 
change in topological phase of the band structure. As in Ref. [26], cyan is negative impedance and magenta is positive; however, the topology changes due to change in $d_{C}$ rather than changes in $\epsilon_{i}$ or $\mu_{i}$. Ideally, the cyan and magenta parts of the impedance map should meet at a point. The reason why they do not is because the map was created using the transmission map. Everywhere the transmission from Fig. 9(a) was less than some selected percentage (say 0.05), that value would be placed in Fig. 9(b) and assigned the correct color according to Eq. (4). Now if the binary $\mathrm{PC}$ is placed next to the quaternary crystal, the new transmission is shown in Fig. 9(c). A topological state can be easily seen in the upper half of the map. For the binary $\mathrm{PC}, d_{C}=0$, so the impedance in the $3^{\text {rd }} \mathrm{PBG}$ is always negative. As $d_{C}$ increases in the other crystal, its impedance eventually flips sign. Therefore, in the region of $d_{C}$ values above the transition, Eq. (3) holds and thus a state appears. This is also clearly shown in Fig. 9(d), in which the imaginary part of Eq. (3) is directly plotted. The state can be seen starting from the crossing point.

In a similar manner to Fig. 9(a), Fig. 10(a) displays the transmission map for the $10^{\text {th }} \mathrm{PBG}$. The main difference now is that there are two points of band gap closure. Unlike the previous case, the gap width undergoes somewhat oscillatory behavior. It can be seen in Fig. 10(b) that the second closing causes the sign of the surface impedance to revert back to the sign it had when $d_{C}=0$. This means that the topological state produced at the interface between the binary and quaternary PCs will vanish before $d_{C}=d_{C}^{\max }$. That state is seen in the transmission map in Fig. 10(c). Lastly, Fig. 10(d), clearly shows the interface state starting at the first crossing point and ending at the second. This means that the state only exists for certain intermediate values of $d_{C}$, for which the superlattice configuration is Fig. 2(b). It is not present for the PC configurations corresponding to the extreme 
values of $d_{C}$ (i.e. Fig. 2(a) for $d_{C}=0$ and Fig. 2(d) for $d_{C}=d_{C}^{\max }$ ). This is in constrast to Fig. 9(d), where the state persisted for $d_{C}^{\max }$.

In Fig. 11(a) and Fig. 11(b), the transmission and impedance maps are shown for a quaternary PC where $n_{C}=\gamma=1.5$. Note that unlike in the previous two examples where PBGs remained open when $d_{C}=d_{C}^{\max }$, all gaps close as $d_{C} \rightarrow 0.5$, leading to a final configuration shown on the RHS of Fig. 2(e). The PC becomes a uniform medium with index, $n_{C}$. With the inclusion of the binary PC on the left hand side (LHS) of the interface, multiple localized states appear, as seen in Fig. 11(c). In total, four are present in the given frequency range, with all them appearing between the first and second gap closing in their respective gap. The states are more clearly defined in Fig. 11(d).

\subsection{Conclusion}

The optical properties of inversion symmetric quaternary PCs have been investigated along with the interface states that appear at the boundary of binary and quaternary PCs. First, the geometry of a quaternary PC was discussed. By inserting two tunable layers into a binary PC to make it quaternary, the rate at which all layers in the unit cell change width with respect to this tunable layer was examined. Depending on the refractive indices, as the tunable layer got large enough, the quaternary PC achieved one of three final configurations. Next, inequalites among $n_{A}, n_{B}$, and $\gamma$ were established that displayed the quaternary PC in a parameter space consisting of dimensions of the layer thickness and refractive index of the tunable layer. As an extension to binary crystals, it was shown that while it was still possible to determine whether the Zak phase of an isolated band is 0 or $\pi$ for a quaternary PC by finding the zeros of $t_{12}$, this must be done numerically; however, unlike for binary PCs, a band can have two frequency crossings, which indicates a Zak phase of 0 for that band. Band crossings were also exam- 
ined in terms of a ratio between two phase coefficents, in this case $\phi_{B} / \phi_{C}$. As this ratio became irrational, the first band crossing occured at increasingly higher band numbers. There also appeared to be two different types of band crossings: one, as stated in previous works, that is described by a simple ratio between the individual optical lengths in the unit cell, but also another class that seems to only be found numercally. Multiple examples of interface state behavior were given for binary/quaternary PC heterostructures. Assuming that when the tunable layer width is 0 the entire structure has the same binary periodicity, interface states only appeared (via topological phase change) between odd and even closing points in all band gaps. This also meant that, especially in higher gaps, there can be interface states that only appear for intermediate values of tunable layer thickness, i.e. states vanish before $d_{C}$ reaches its maximum value, causing the quaternary PC to become a (different) binary one. 


\begin{tabular}{|c|c|c|}
\hline \multicolumn{3}{|c|}{ PC Parameters } \\
\hline \multicolumn{3}{|c|}{$\gamma=3 / 2$} \\
\hline Layer & Relative Permittivity, $\varepsilon$ & Relative Permeability, $\mu$ \\
\hline A & 6 & 1 \\
\hline B & 1 & 1 \\
\hline
\end{tabular}

(a)

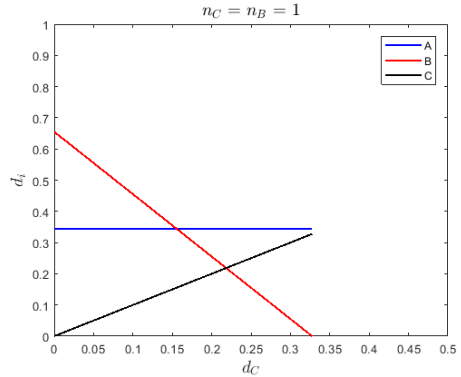

(c)

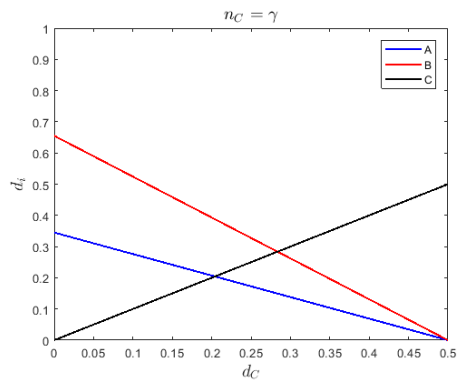

(e)

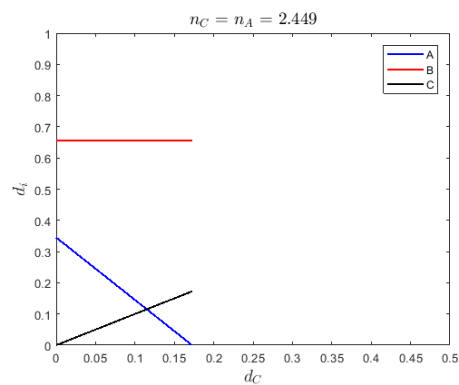

(g)

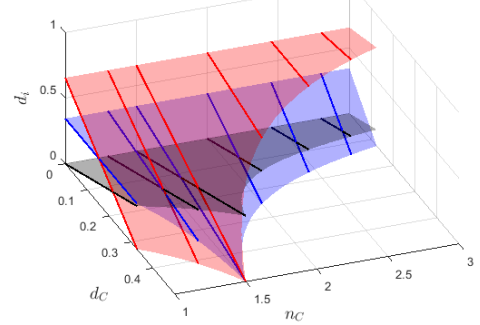

(b)

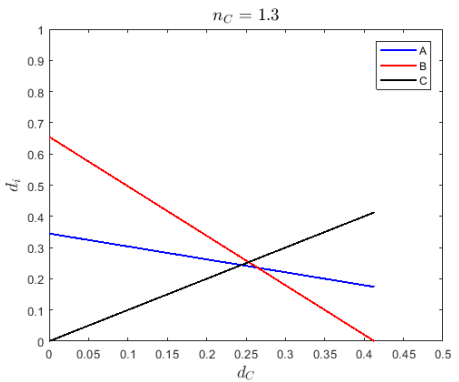

(d)

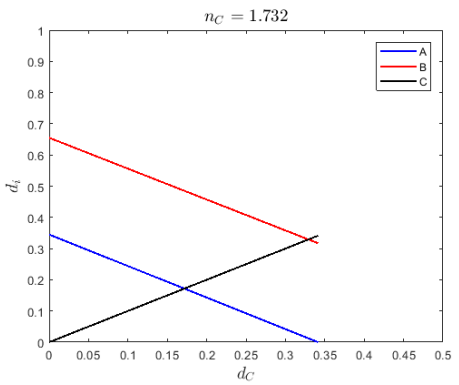

(f)

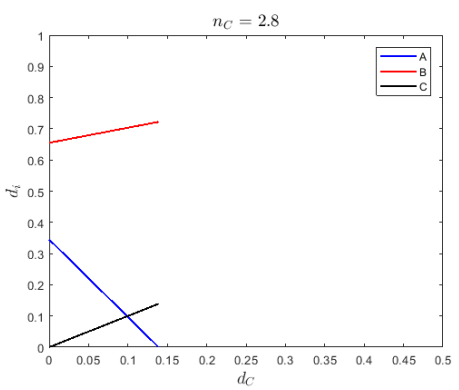

(h)

Figure 3: (a) $\gamma, n_{A}$, and $n_{B}$ are fixed in the system. (b) Surfaces are used to show how the relative thicknesses of layers A (blue), B (red), and C (black) change according to Eqs. (5) and (6). Six different trios of lines of constant $n_{C}$ are highlighted. (c)-(h) Those six trios are plotted sepa rately as profiles with the value of $n_{C}$ displayed above each plot. 


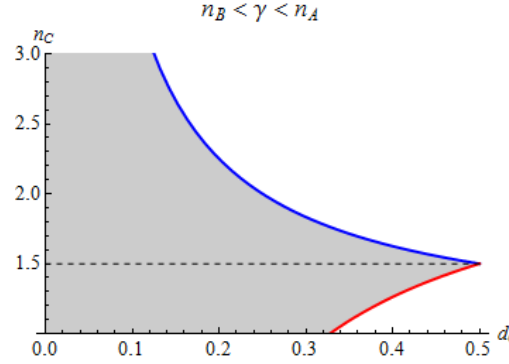

(a)

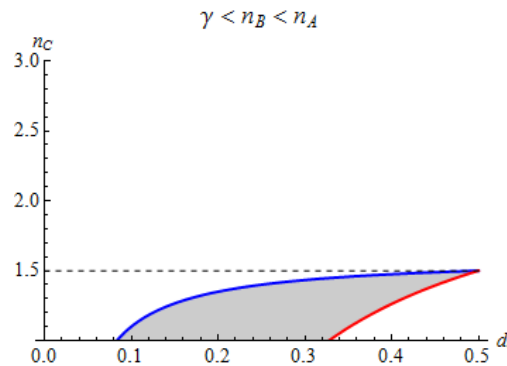

(c)

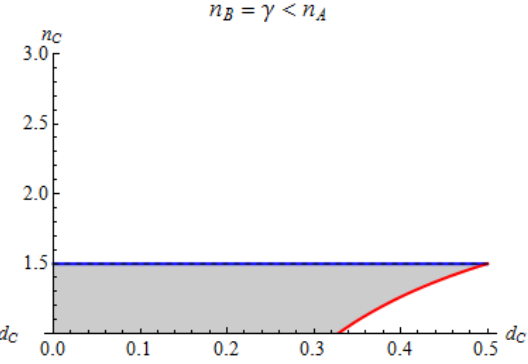

(b)

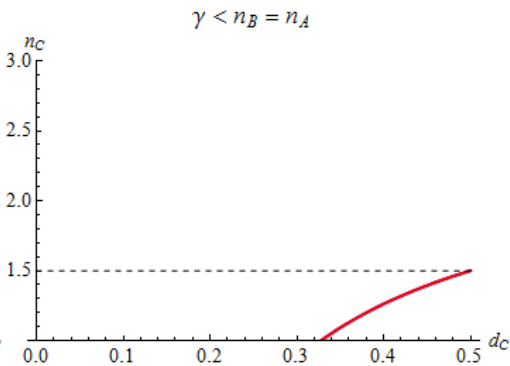

(d)

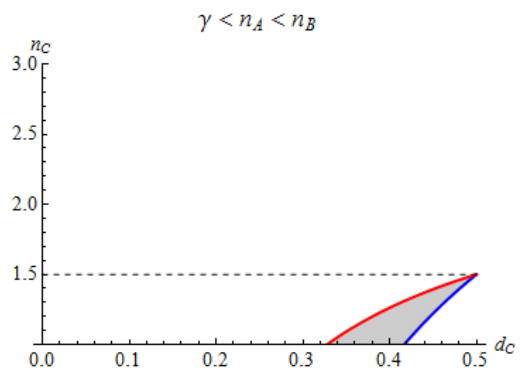

(e)

Figure 4: (a)-(e) The $\left(d_{C}, n_{C}\right)$ parameter space is displayed. The PC can only exist in the shaded areas where $d_{A}, d_{B}, d_{C}$ are all non-negative. $\gamma$ and $n_{A}$ are constants, with $\gamma<n_{A}$ and $n_{B}$ is free to vary. The dashed line is $n_{C}=\gamma$. The blue and red lines are Eq. (7) and Eq. (8), respectively. In (d), the shaded region vanishes, since the curves overlap. 


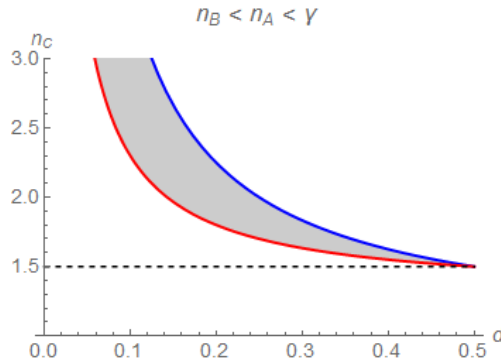

(a)

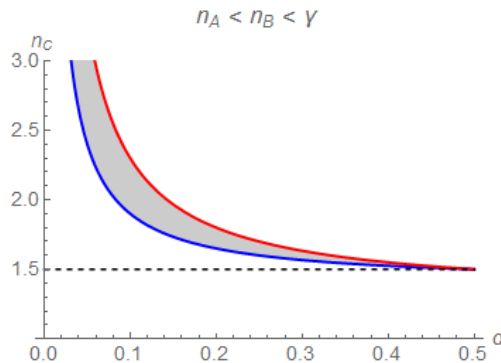

(c)

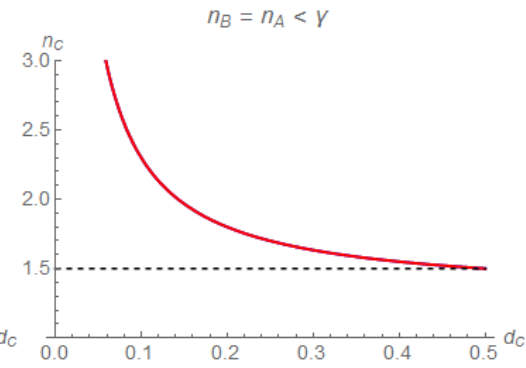

(b)

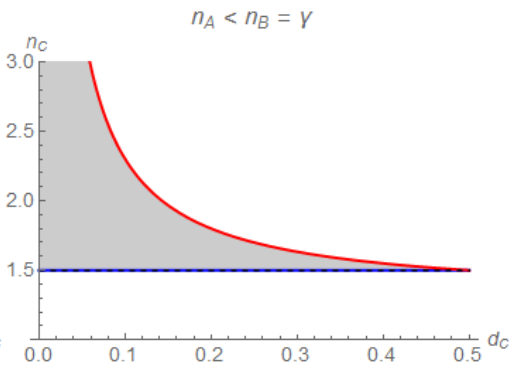

(d)

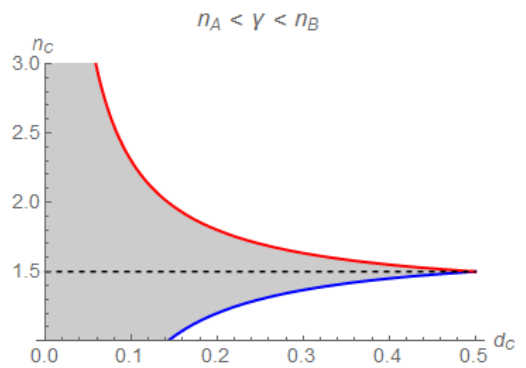

(e)

Figure 5: (a)-(e) Similar to Fig. 4 , the $\left(d_{C}, n_{C}\right)$ parameter space is displayed. The PC can only exist in the shaded areas where $d_{A}, d_{B}, d_{C}$ are all non-negative. $\gamma$ and $n_{A}$ are constants, with $\gamma>n_{A}$ and $n_{B}$ is free to vary. The dashed line is $n_{C}=\gamma$. The blue and red lines are Eq. (7) and Eq. (8), respectively. In (b), the shaded region vanishes, since the curves overlap 


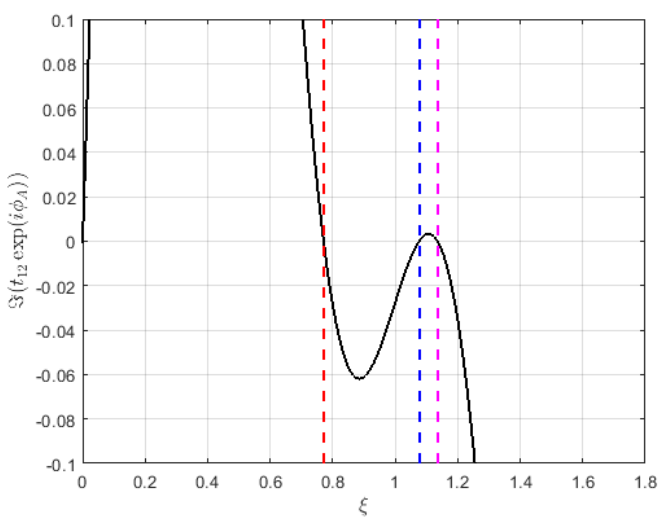

(a)

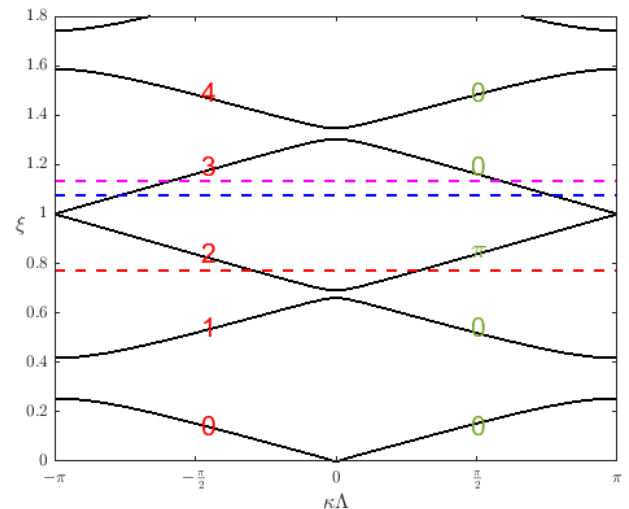

(b)

Figure 6: System Parameters: $d_{A}=0.2005, \epsilon_{A}=1, \mu_{A}=6, d_{B}=0.5135, \epsilon_{B}=1$, $\mu_{B}=1, d_{C}=0.143, \epsilon_{C}=1, \mu_{C}=3$ (a) $\operatorname{Im}\left(t_{12} \exp \left(i \phi_{A}\right)\right)$ is plotted with respect to frequency, $\xi$. The zeros are displayed as vertical dashed lines. (b) Band structure is plotted. Those three frequencies are superimposed on the plot. The band numbers are displayed in red and the Zak phases in green. There is a very thin PBG at $\xi \approx 0.9996$. 


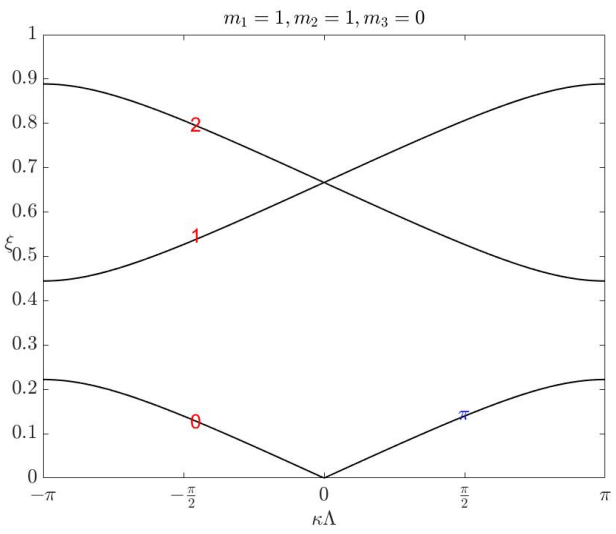

(a)

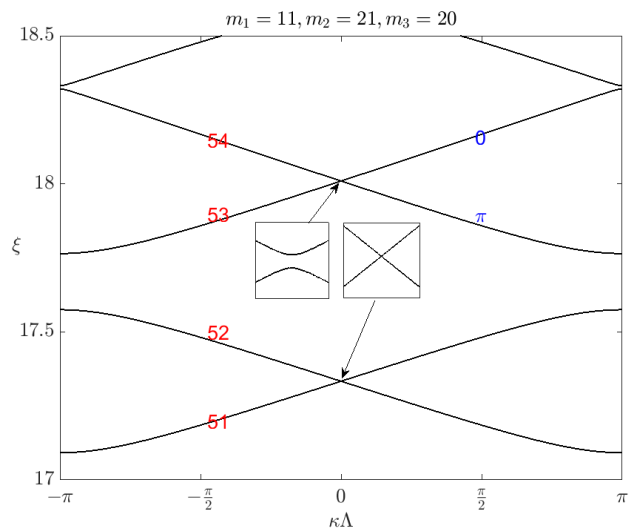

(c)

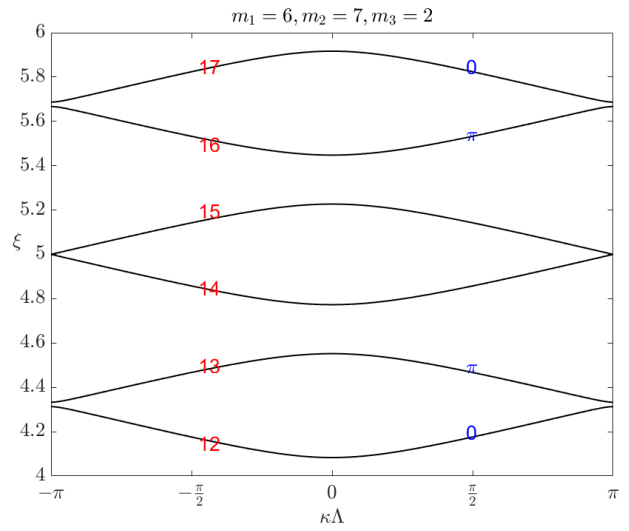

(b)

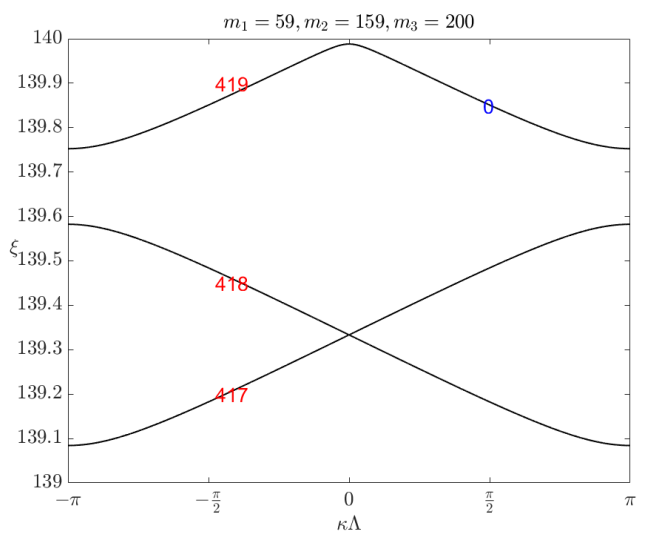

(d)

Figure 7: Band crossing examples for $\phi_{B}=M \phi_{C}$ and $l=1$ (lowest crossing) For each case, $\gamma=1.5, n_{A}=3, n_{B}=1, n_{C}=2$. Red values are band numbers and blue values are Zak phases. Only isolated bands have a Zak phase. (a) $M=\infty$ This is just a binary PC. The first crossing occurs between bands 1 and 2. (b) $M=7$ The first crossing occurs between bands 14 and 15 . (c) $M=2.1$ The first crossing occurs between bands 51 and 52. The two inserts show that it can be difficult to distingish a crossing from a thin gap solely by eye. (d) $M=1.59$ The first crossing occurs between bands 417 and 418 . 


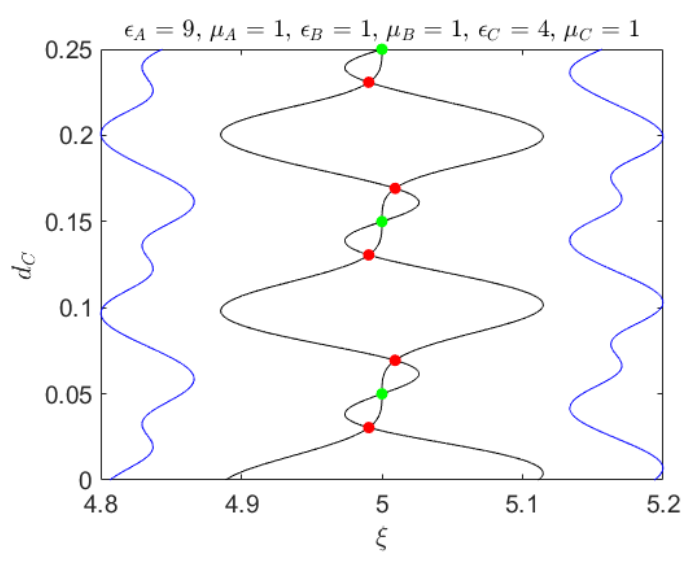

(a)

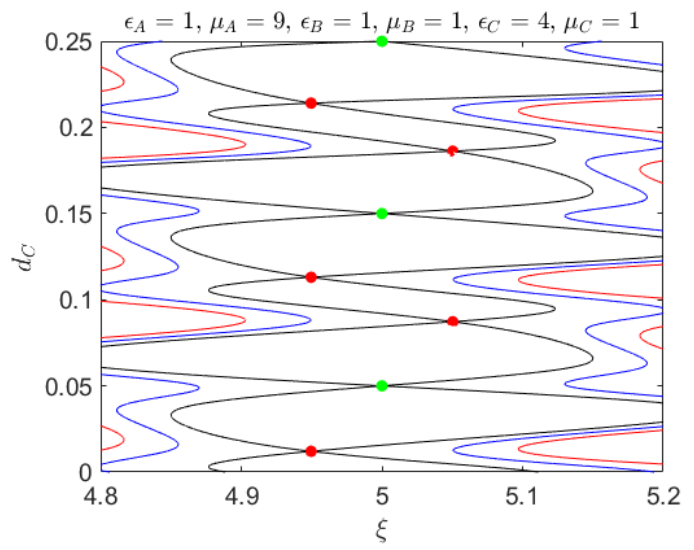

(b)

Figure 8: Locations of the PBG closing points are shown in $\left(\xi, d_{C}\right)$ parameter space. Blue curve are $t_{11}+t_{22}=0$, black curve are $t_{11}+t_{22}=-2$, and red curves are $t_{11}+t_{22}=2$. Green dots are gap closings described by Eq. (23); red dots are closings found graphically or numerically. 


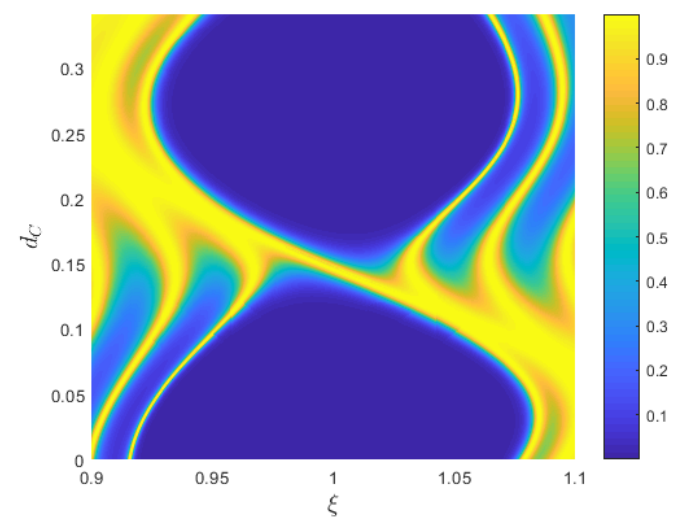

(a)

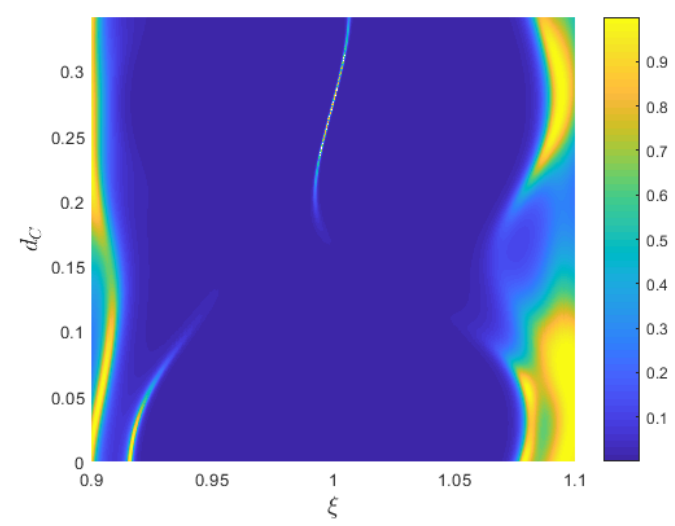

(c)

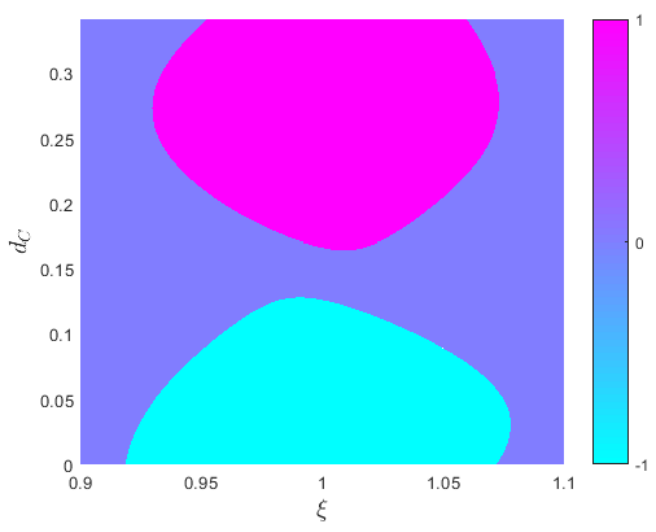

(b)

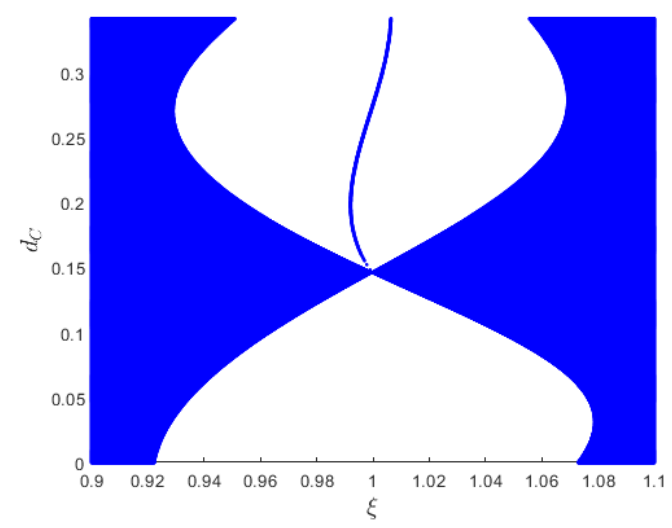

(d)

Figure 9: (a) Transmission spectrum about the $3^{\text {rd }} \mathrm{PBG}$ for inversion symmetric quaternary $\mathrm{PC}$ with parameters from Fig. 3(a) as well as $\epsilon_{C}=3, \mu_{C}=1$. There is one region where the $\mathrm{PBG}$ closes then reopens, at $\left(\xi, d_{C}\right) \approx(0.9996,0.1461)$. Ten unit cells are used. (b) Sign $\left(\zeta^{(3)}\right)$ is displayed as described by Eq. (4). Colors are kept consistent with Ref. [26].The impedance switches sign after the gaps reopen. (c) Transmission spectrum for combined system of binary and quaternary PCs. For $d_{C}=0$, the system is described by Fig. 2(a). For $d_{C}=d_{C}^{\max }=0.341$, it is described by Fig. 2(d). For $0<d_{C}<d_{C}^{\max }$, the system is described by Fig. 2(b). Five unit cells are used for each PC. Note the topological state after the gap reopens. (d) The state is clearly shown by plotting the implicit equation, $Z_{\text {left }}(\xi)+Z_{\text {right }}\left(\xi, d_{C}\right)=0$, where each term is described by Eq. (16). 


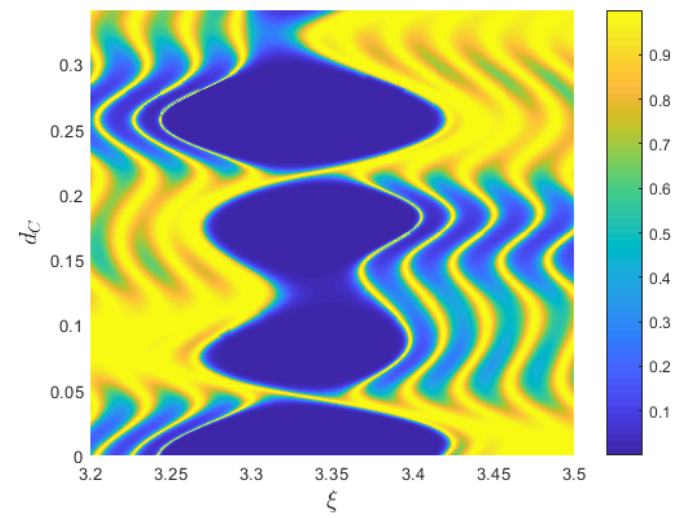

(a)

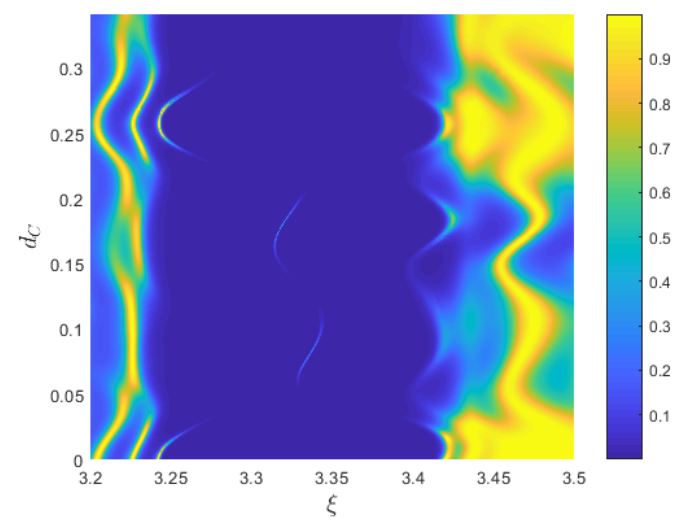

(c)

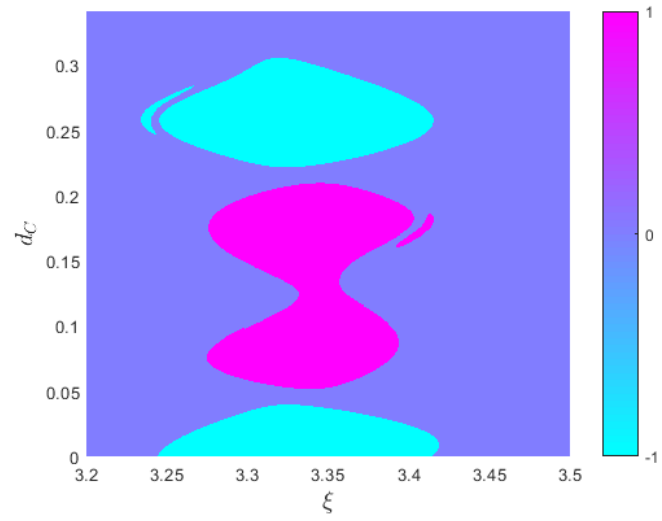

(b)

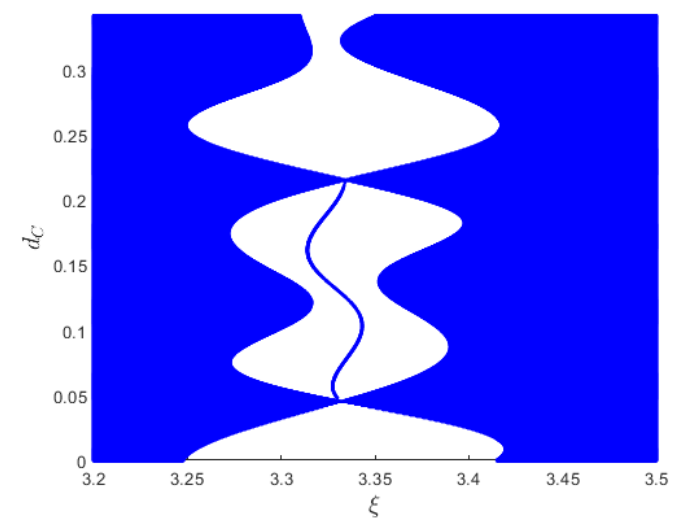

(d)

Figure 10: (a)-(d) Similar to Fig. 9 except the plots focus on the $10^{\text {th }}$ PBG. There are two regions where the PBG closes then reopens, at $\left(\xi, d_{C}\right) \approx(3.332,0.04508)$ and $\left(\xi, d_{C}\right) \approx(3.334,0.2151)$. 


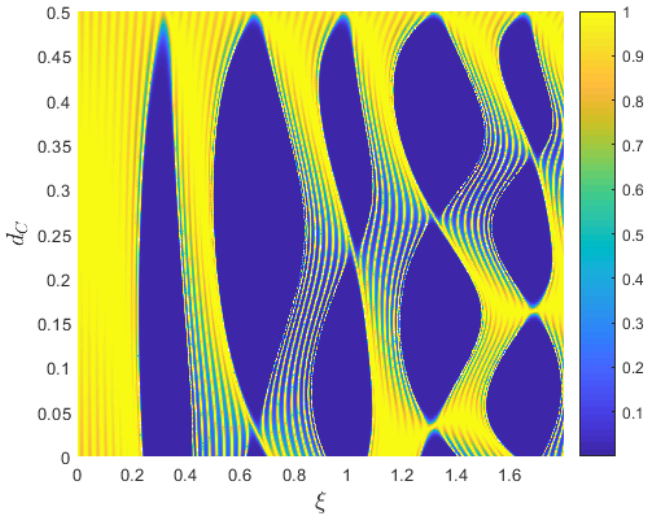

(a)

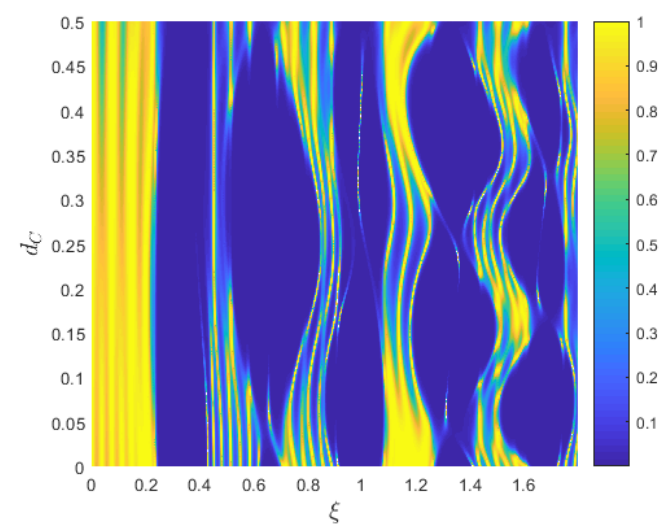

(c)

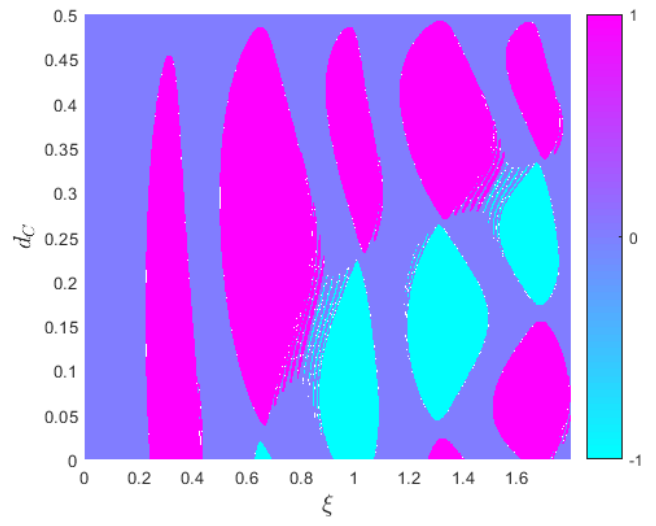

(b)

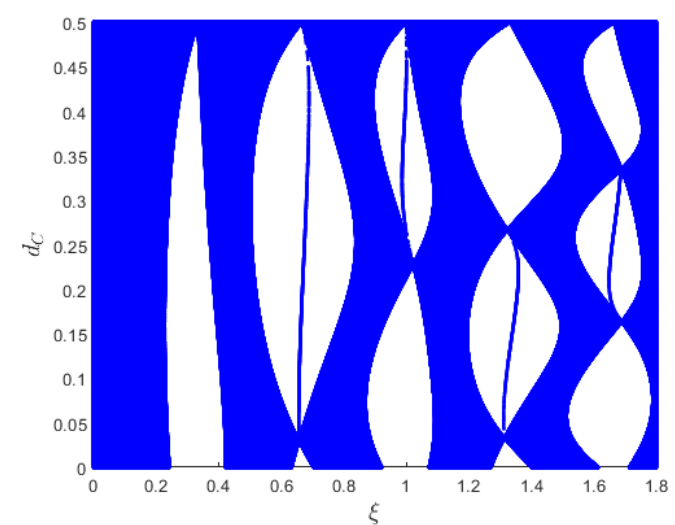

(d)

Figure 11: (a)-(d) Similar to Fig. 9 except $\epsilon_{C}=1$ and $\mu_{C}=2.25$. PBGs 1-5 are shown 


\subsection{References \\ List of References}

[1] J. D. Joannopoulos, R. D. Meade, J. N. Winn, and S. G. Johnson, Photonic Crystals: Molding The Flow of Light, (Princeton University, Princeton, NJ, 2008).

[2] F. Bloch, Über die Quantenmechanik der Elektronen in Kristallgittern, Z. Phys. 52, 555 (1929).

[3] J. W. Strutt, On the reflection of light from a regularly stratified medium, Proceedings of the Royal Society of London. Series A, Containing Papers of a Mathematical and Physical Character 93, 565 (1917).

[4] E. Yablonovitch, Inhibited Spontaneous Emission in Solid-State Physics and Electronics, Phys. Rev. Lett. 58, 2059 (1987).

[5] E. Yablonovitch, Photonic band-gap structures, J. Opt. Soc. Am. B 10, 283 (1993).

[6] S. John, Strong localization of photons in certain disordered dielectric superlattices, Phys. Rev. Lett. 58, 2486 (1987).

[7] M. C. Netti, M. D. B. Charlton, G. J. Parker, and J. J. Baumberg, Visible photonic band gap engineering in silicon nitride waveguides, Appl. Phys. Lett. 76, 991 (2000).

[8] J. C. Knight, T. A. Birks, P. St. J. Russell, and D. M. Atkin, All-silica singlemode optical fiber with photonic crystal cladding, Opt. Lett. 21, 1547 (1996).

[9] H. Vinck-Posada, and F. A. Segovia-Chaves, Transmittance spectra in onedimensional dielectric photonic crystals with defects, J. Phys: Conference Series 792, 012008 (2017).

[10] H. Xiao-Qin, and C. Yi-Ping, Degeneracy and Split of Defect States in Photonic Crystals, Chin. Phys. Lett. 20, 1721 (2003).

[11] M. Florescu, S. Torquato, and P. J. Steinhardt, Complete band gaps in twodimensional photonic quasicrystals, Phys. Rev. B 80, 155112 (2009).

[12] X. Jiang, Y. Zhang, S. Feng, K. C. Huang, Y. Yi, and J. D. Joannopoulos, Photonic band gaps and localization in the Thue-Morse structures, Appl. Phys. Lett. 86, 201110 (2005).

[13] H. Lei, J. Chen, G. Nouet, S. Feng, Q. Gong, and X. Jiang, Photonic band gap structures in the Thue-Morse lattice, Phys. Rev. B 75, 205109 (2007). 
[14] A. N. Poddubny, and E. L. Ivchenko, Photonic quasicrystalline and aperiodic structures, Physica E: Low-dimensional Systems and Nanostructures 42, 1871 (2010).

[15] S. Torquato, F. H. Stillinger, Local density fluctuations, hyperuniformity, and order metrics, Phys. Rev. E 68, 041113 (2003).

[16] W. Man, M. Florescu, E. P. Williamson, Y. He, S. R. Hashemizad, B. Y. C. Leung, D. R. Liner, S. Torquato, P. M. Chaikin, and P. J. Steinhardt, Isotropic band gaps and freeform waveguides observed in hyperuniform disordered photonic solids, National Academy of Sciences 110, 15886 (2013).

[17] W. Man, M. Florescu, K. Matsuyama, P. Yadak, S. Torquato, P. Steinhardt, and P. Chaikin, "Experimental observation of photonic bandgaps in hyperuniform disordered material," in Conference on Lasers and Electro-Optics 2010, OSA Technical Digest (CD) (Optical Society of America, 2010), paper CThS2.

[18] W. Man, M. Florescu, K. Matsuyama, P. Yadak, G. Nahal, S. Hashemizad, E. P. Williamson, P. Steinhardt, S. Torquato, and P. M. Chaikin, Photonic band gap in isotropic hyperuniform disordered solids with low dielectric contrast, Opt. Express 21, 19972 (2013).

[19] A. V. Kavokin, I. A. Shelykh, and G. Malpuech, Lossless interface modes at the boundary between two periodic dielectric structures, Phys. Rev. B 72, 233102 (2005).

[20] I. Tamm, Über eine mögliche Art der Elektronenbindung an Kristalloberflächen, Z. Phys. 76, 849 (1932).

[21] A. P. Vinogradov, A. V. Dorofeenko, S. G. Erokhin, M. Inoue, A. A. Lisyansky, A. M. Merzlikin, and A. B. Granovsky, Surface state peculiarities in onedimensional photonic crystal interfaces, Phys. Rev. B 74, 045128 (2006).

[22] T. Goto, A. V. Dorofeenko, A. M. Merzlikin, A. V. Baryshev, A. P. Vinogradov, M. Inoue, A. A. Lisyansky, and A. B. Granovsky, Optical Tamm States in One-Dimensional Magnetophotonic Structures, Phys. Rev. Lett. 101, 113902 (2008).

[23] X. Kang, W. Tan, Z. Wang, and H. Chen, Optic Tamm states: The Blochwave-expansion method, Phys. Rev. A 79, 043832 (2009).

[24] A. P. Vinogradov, A. V. Dorofeenko, A. M. Merzlikin, and A. A. Lisyansky, Surface states in photonic crystals, Sov. Phys.-Usp 53, 243 (2010).

[25] W. Shockley, On the Surface States Associated with a Periodic Potential, Phys. Rev. 56, 317 (1939). 
[26] M. Xiao, Z. Q. Zang, and C. T. Chan, Surface Impedance and Bulk Band Geometric Phases in One-Dimensional Systems, Phys. Rev. X 4, 021017 (2014).

[27] J. Zak, Berry's phase for energy bands in solids, Phys. Rev. Lett. 62, 2747 (1989).

[28] I. Nusinsky, and A. A. Hardy, Band-gap analysis of one-dimensional photonic crystals and conditions for gap closing, Phys. Rev. B 73, 125104 (2006).

[29] W. S. Gao, M. Xiao, C. T. Chan, and W. Y. Tam, Determination of Zak phase by reflection phase in 1D photonic crystals, Opt. Lett. 40, 5259 (2015).

[30] W. S. Gao, M. Xiao, B. Chen, E. Y. B. Pun, C. T. Chan, and W. Y. Tam, Controlling interface states in $1 D$ photonic crystals by tuning bulk geometric phases, Opt. Lett. 42, 1500 (2017).

[31] Q. Wang, M. Xiao, H Liu, S. Zhu, and C. T. Chan, Measurement of the Zak phase of photonic bands through the interface states of a metasurface/photonic crystal, Phys. Rev. B 93, 041415(R) (2016).

[32] K. H. Choi, C. W. Ling, K. F. Lee, Y. H. Tsang, and K. H. Fung, Simultaneous multi-frequency topological edge modes between one-dimensional photonic crystals, Opt. Lett. 41, 1644 (2016).

[33] P. A. Kalozoumis, G. Theocharis, V. Achilleos, S. Félix, O. Richoux, and V. Pagneux, Finite-size effects on topological interface states in one-dimensional scattering systems, Phys. Rev. A 98, 023838 (2018).

[34] W. Gao, X. Hu, C. Li and J. Yang, Z. Chai, J. Xie, and Q. Gong, Fanoresonance in one-dimensional topological photonic crystal heterostructure, Opt. Express 26, 8634 (2018).

[35] H. Deng, X. Chen, N. C. Panoiu, F. Ye, Topological surface plasmons in superlattices with changing sign of the average permittivity, Opt. Lett. 41, 4281 (2016).

[36] L. Ge, L. Liu, M. Xiao, G. Du, L. Shi, D. Han, C. T. Chan, and J. Zi, Topological phase transition and interface states in hybrid plasmonic-photonic systems, J. Opt. 19, 06LT02 (2017).

[37] L. Wang, W. Cai, M. Bie, X. Zhang, and J. Xu, Zak phase and topological plasmonic Tamm states in one-dimensional plasmonic crystals, Opt. Express 26, 28963 (2018).

[38] B. Midya, and L. Feng, Topological multiband photonic superlattices, Phys. Rev. A 98, 043838 (2018). 
[39] Q. Li, and X. Jiang, Singularity induced topological transition of different dimensions in one synthetic photonic system, Opt. Commun. 440, 32 (2019).

[40] Q. Li, Y. Zhang, and X. Jiang, Two classes of singularities and novel topology in a specially designed synthetic photonic crystals, Opt. Express 27, 4956 (2019).

[41] A. Yariv, and P. Yeh, Optical Waves in Crystals: Propagation and Control of Laser Radiation, (John Wiley \& Sons, 1984). 


\title{
MANUSCRIPT 2
}

\section{Optical States in a 1-D Superlattice with Multiple Photonic Crystal Interfaces}

\author{
Nicholas J Bianchi and Leonard M Kahn \\ University of Rhode Island \\ Physics Department
}

This manuscript has been submitted to IOP Journal of Optics. 


\section{$2.1 \quad$ Abstract}

Interface states in a 1-D photonic crystal heterostructure with multiple interfaces are examined. The heterostructure is a periodic network consisting of two different photonic crystals. In addition, the two crystals themselves are periodic, with one being made of alternating binary layers and the other being a quaternary crystal with a tunable layer. The second crystal can thus be smoothly transformed from one binary crystal to another. All individual photonic crystals in the superstructure have symmetric unit cells, as well as identical periods and optical path lengths. Therefore, as the tunable layer in the quaternary crystal expands, other layers will shrink. It is found that the behavior of the localized modes in the band gaps is dependent on whether there is an even or odd number of interfaces in the heterostructure. With certain sequences of all dielectric photonic crystals, topological states are shown to split in two, whereas for other heterostructures they are shown to vanish. Additional resonant modes appear depending on how many crystals are in the heterostructure. If the tunable layer is frequency dependent, the band gap can still support topological/resonant modes with some band gaps even supporting two separate groups.

\subsection{Introduction}

A photonic crystal (PC) is a periodic array of dielectrics and/or conductors used to scatter light $[1,2]$. In a similar manner to how semiconductors control the passage of electrons, $\mathrm{PCs}$ possess passbands which allow photons in certain frequency ranges to propagate through the crystal and photonic band gaps (PBGs), which inhibit photon flow, producing regions of suppressed transmission. The existence of these pass and stop bands are governed by Bloch's Thereom. Photonic heterostructure devices are comprised of multiple periodic components that can produce transmission properties and field localization not seen in isolated crystals 
$[3,4]$. Heterostructures with a single PC interface have been extensively studied. Examples of localized behavior are the surface or interface modes, also known as optical Tamm [5] states (OTSs). These modes can exist at a boundary only if their field amplitudes decay away as the distance from the boundary increases in either direction. This means the wavevectors must be imaginary. In the case of a PC, this occurs if the mode is trying to travel through a PBG. These modes have been found in a variety of photonic structures including 1-D [8, 6, 7, 9] and 2-D [10] $\mathrm{PC}$ interfaces, air-PC surfaces at oblique angles [11], and PCs bordering media with a graded refractive index [12]. Tamm states have also been investigated in various systems containing a $\mathrm{PC}$ with a tunable cap layer adjacent to a uniform medium. Examples include PCs containing superconducting layers [13], systems containing metamaterials, both the PC layers $[14,15]$ and the uniform medium [16], and systems with liquid crystal [17] and chiral [18] cap layers. Note that in Ref. [11], despite the PC being adjacent to a uniform medium with positive dielectric constant, surface modes can still form due to total internal reflection. The component of the wavevector parallel to the boundary, $k_{\|}$, is large enough to cause the normal component, $k_{\perp}$ to become imaginary.

$$
k_{\perp}=\sqrt{k^{2}-k_{\|}^{2}}
$$

A varient of OTSs are the Tamm plasmon-polaritons (TPP) formed at a boundary between a metal and a PC $[19,20,21]$. In order for a TPP to form, the condition,

$$
r_{\text {metal }} r_{P C}=1
$$

must be satisfied. The reflection coefficent $r_{\text {metal }}$ describes the amplitude of the electric field, incident from the PC side of the interface, reflecting off the metallic surface. In the same manner, $r_{P C}$ describes the electric field amplitude from a wave incident from the metallic side reflecting off the PC surface of the interface. 
In the case described in Ref. [19], the TPP is excited at a frequency below the plasma frequency of the metal, implying that $r_{\text {metal }}=-1$. Therefore, to ensure that Eq. 25 remains satisifed, $r_{P C}=-1$, implying that the higher index material in the PC should be adjacent to the metal. In Ref. [20], the plasmon is produced above the plasma frequency. Since the permittivity of the metal is now positive, $r_{\text {metal }}$ flips sign. For the state to exist now, the sign of $r_{P C}$ must also flip, meaning that, in the PC, the low index material is adjacent to the material. Similar to Ref. [11], the state is supported on the metallic side by total internal reflection.

If an interface is generated between two PCs with symmetric unit cells, localized states at the boundary can form that are governed by the bulk band structure of the two crystals. These states are referred to as topological interface states. Xiao et.al. [22] showed that their existence in a PBG can be predicted by ensuring that the imaginary parts of the surface impedances for the two crystals sum to zero in the selected gap. Their work established a relation between the sign of the impedance $Z$ for a PBG and the sum of all Zak [23] phases, $\theta_{m}^{z a k}$, below the gap, where $m$ denote the (isolated) bands,

$$
\operatorname{sign}\left(\operatorname{Im}\left(Z^{(n)}\right)\right)=(-1)^{n+l} \exp \left(i \sum_{m=1}^{n} \theta_{m}^{z a k}\right)
$$

In Eq. 26, $n$ is the PBG where the impedance is calculated and $l$ denotes the number of points where two bands cross below band gap $n$. Due to the PC unit cells possessing inversion symmetry, all Zak phases can only take on the values of $\pi$ or 0 [23], and thus provide a useful measure for identifying topological states. Band gap $n$ contains a topological state if $Z_{L}+Z_{R}=0$, where the subscripts indicate the PCs to the left/right of the interface. Through control of $\theta_{m}^{z a k}$, topological states have been demonstrated in both 1-D [24, 25] and 2-D [26] systems.

Heterostructures with multiple $\mathrm{PC} / \mathrm{PC}$ or $\mathrm{PC} /$ metallic interfaces have more degrees of freedom due to the increased number of tunable parameters, as compared 
to a single interface system, leading to a much richer display of resonant states. Through the control of parameters within the heterostructure, several examples of coupling between resonant states have been demenstrated [21, 27, 28, 29, 30, 31]. As an extension to the work in Ref. [32], the behavior of interface states is investigated in a heterostructure consisting of alternating binary and quaternary PCs. If there is an equal number of binary and quaternary crystals in the stucture, then there is an odd number of crystal interfaces. In this case, if the first PC in the heterostructure is binary (quaternary), then, after the alternating pattern, the last will be quaternary (binary). The orginal topological state from the two crystal hetrostructure remains but is now accompanied by a sequence of resonant states on either side. The total number of states, including the original, is equal to the number of interfaces. For an even number of interfaces, there are two possible configurations. One possibility is to have the first and last PC of the structure be binary. In this case, it is found that the orginal topological state vanishes while the resonant states remain. The other case is to have the first and last crystals be quaternary. With only a single binary PC sandwiched between two quaternary PCs, the topological state splits. If more layers are added in this scenario, keeping the two ends quaternary, the split state is joined by resonant states.

\subsection{Methods}

Our work was conducted using transfer matrix method (TMM) [33]. Keeping with Ref. [32], all variables are made dimensionless for convenience. The lengths of the individual PC layers, $l_{i}$, are scaled to the unit cell period, $\Lambda: d_{i}=l_{i} / \Lambda$ and are such that $\Lambda$ and the optical path, $\Gamma$, for a unit cell are constant. In the heterostructure, shown as the middle image in Fig. 12, the periods for all the individual PCs are equal, as are the optical paths. The binary PCs are the gray regions and the quaternary PCs are the light blue regions. Since there is no fixed 


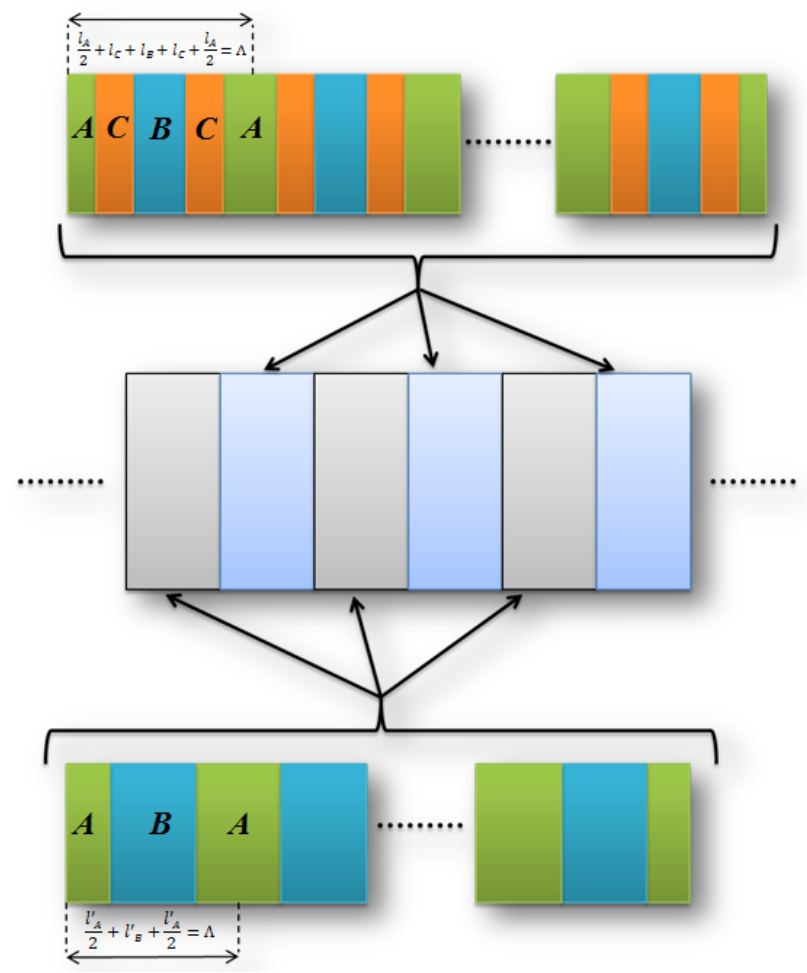

Figure 12: Schematic of PCs and overall heterostructure. The heterostructure is displayed in the middle as repeating light grey and blue "slabs". Each of these "slabs" represent entire photonic crystals. The grey slabs represent binary PCs, displayed at the bottom. In the bottom diagram, dark green is layer $A$ and dark blue is layer $B$. The vertical dashed lines and double arrow show one unit cell. The primes indicate that the individual layer lengths are different from those of the quaternary PC. Note that the PC is capped on both sides with a half-width of layer $A$, making the unit cells symmetric. The top diagram shows the quaternary $\mathrm{PC}$, which is represented in the middle picture as the light blue regions. In the quaternary PC, layers $A$ and $B$ are the same material as in the binary crystal. Layer $C$ is orange. As in the binary case, the symmetric unit cell is displayed by the double arrow.

length scale, we set $\gamma=\Gamma / \Lambda$. For the quaternary PC, shown at the top of Fig. 12, the widths of layers $A$, in green, and $B$, in blue, can be expressed in terms of a free parameter, the width of the introduced layer, $d_{C}$, in orange, [32],

$$
d_{A}=\frac{\gamma-n_{B}-2\left(n_{C}-n_{B}\right) d_{C}}{n_{A}-n_{B}}
$$




$$
d_{B}=\frac{\gamma-n_{A}-2\left(n_{C}-n_{A}\right) d_{C}}{n_{B}-n_{A}}
$$

Note that $d_{C}$ can only take on values in which both Eqs. 27 and 28 are nonnegative. When $d_{C}$ reaches its maximum, the quaternary PC will become binary

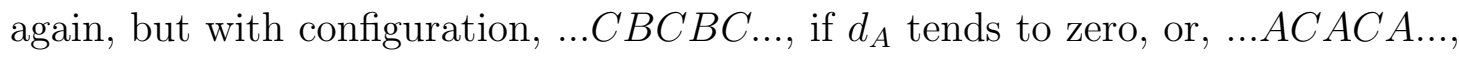
if $d_{B}$ tends to zero. For the special case, $\gamma=n_{C}$, both $d_{A}$ and $d_{B}$ will be zero when $d_{C}$ reaches its maximum; this will result in a uniform layer $C$. The lengths of the layers in the binary PC, displayed at the bottom of Fig. 12, are simply Eqs. 27 and 28 but with $d_{C}=0$ and thus do not change. The index of refraction of layer $j$ is $n_{j}^{2}=\epsilon_{j} \mu_{j}$, where $\epsilon_{j}$ and $\mu_{j}$ are the (relative) permittivites and permeabilites. In the binary and quaternary crystals, the $n_{A}$ 's are the same and the $n_{B}$ 's are the same, although $n_{A} \neq n_{B}[32]$.

For the system described in Fig. 12, we only consider an electric field incident from the left, $E_{1+}$. The reflected field is $E_{1-}$ and the field that is transmitted through the entire structure is $E_{(N+1)+}^{\prime}$. To compute the transmission spectra for the system, first we must construct the transfer matrix, $\mathbf{M}$, from the individual interface matrices, $\mathbf{I}_{j}$, and propagation matrices, $\mathbf{P}_{j}$, where the index, $j$, specifies the layer in question [34],

$$
\begin{gathered}
\mathbf{I}_{j}=\frac{1}{\tau_{j}}\left(\begin{array}{cc}
1 & r_{j} \\
r_{j} & 1
\end{array}\right) \\
\mathbf{P}_{j}=\left(\begin{array}{cc}
e^{2 \pi i n_{j} d_{j} \xi} & 0 \\
0 & e^{-2 \pi i n_{j} d_{j} \xi}
\end{array}\right)
\end{gathered}
$$

where $r_{j}$ and $\tau_{j}$ are the reflection and transmission coefficients, respectively,

$$
\begin{aligned}
& r_{j}=\frac{\mu_{j+1} n_{j}-\mu_{j} n_{j+1}}{\mu_{j+1} n_{j}+\mu_{j} n_{j+1}} \\
& \tau_{j}=\frac{2 \mu_{j+1} n_{j}}{\mu_{j+1} n_{j}+\mu_{j} n_{j+1}}
\end{aligned}
$$


In scaled variables, the phase argument, $i k_{j} l_{j}$ becomes $2 \pi i n_{j} d_{j} \xi$. The frequency, $f$, becomes $\xi=f \Lambda / c_{0}$, where $c_{0}$ is the speed of light in vacuum. The incident and scattered field are related by,

$$
\left(\begin{array}{c}
E_{1+} \\
E_{1-}
\end{array}\right)=\left(\begin{array}{ll}
M_{11} & M_{12} \\
M_{21} & M_{22}
\end{array}\right)\left(\begin{array}{c}
E_{(N+1)+}^{\prime} \\
0
\end{array}\right)
$$

where,

$$
\mathbf{M}=\left(\begin{array}{ll}
M_{11} & M_{12} \\
M_{21} & M_{22}
\end{array}\right)=\prod_{j=1}^{N} \mathbf{I}_{j} \mathbf{P}_{j} \mathbf{I}_{N+1}
$$

The transmitted power is calculated via,

$$
T\left(\xi, d_{C}\right)=\left|\frac{1}{M_{22}}\right|^{2}
$$

\subsection{Results}

In our first investigation, all layers of the heterostructures are assumed to be lossless dielectrics with no material dispersion. For both the binary and quaternary PCs, $\epsilon_{A}=6, \epsilon_{B}=\mu_{A}=\mu_{B}=1$. In the quaternary PC, $\epsilon_{C}=3$ and $\mu_{C}=1$. For simplicity, all PCs are given the same number of unit cells, $N_{\Lambda}$, periods and optical paths. In the following systems, $N_{\Lambda}=4$ and $\gamma=1.5$. With $d_{C}$ as a free parameter, $d_{A}$ and $d_{B}$ of the quaternary PC are described by Eqs. $27 \& 28$. Since the physics of interface states is valid in any PBG, we will restrict ourselves to the $3^{\text {rd }}$ one since this is the lowest gap that produces such states with the described parameters. For convenience, when describing individual PCs of the heterostructures, we will use $b$ for binary PC and $q$ for quaternary PC.

Transmission through the heterosturcture depends on the specific configuration of the binary and quaternary PCs. Fig. 13 displays nine different transmission examples. In Figs. 13(a)-13(c), the stucture is sandwiched between two binary PCs, while in Figs. 13(d)-13(f), the two endlayers are quaternary PCs. In both cases, the number of interfaces between individual PCs from left to right is 2,4 , and 6 . 
Since there is an even number of interfaces in the heterostructure, a single topological peak (Fig. 13(g)) is absent, even though Eq. 26 states that there is a change in the sign of surface impedance between the binary and quaternary components as $d_{C}$ increases from 0 to 0.341 . For the transmission maps in the top row, the heterostructure has the form $b q b, b q b q b$ and $b q b q b q b$. It can be seen that the state in a single interface system splits into two sets of resonances, with one set below the original frequency and the other above. At $d_{C}=0$, all these states exist as pass band modes; however, as $d_{C}$ increases, they begin to wander into the band gap. As these resonant states appear at all values of $d_{C}$, they are not topological in nature, although after the impedance for the quaternary layers flips sign (see Eq. 26), they appear to cluster together and move in a similar manner to the topological state in the single binary-quaternary interface heterostructure. The transmission for all these states remains at unity for all values of $d_{C}$.

When the heterostructure changes from $b q b$ to $b q b q b$, the two states themselves split into pairs such that these pairs (Fig. 13(b)) each have a higher and lower frequency state relative to their respective states in Fig. 13(a). This splitting is illustrated in Fig. 14. A horizontal slice of Fig. 13(b) at $d_{C}=0.25$ is considered, except now the states are plotted for varying thickness of the middle cystral. Each binary and quaternary represent 4 unit cells; $b / 2$ represents 2 unit cells. In Fig. 14(a), we see two distant edge states (blue) in the absence of a middle $b$ : $b q q b$. To see the two interface states, though, we must zoom into the cluttered middle region. These interface states are clearly seen in Fig. 14(b). When two binary unit cells are inserted in the center of the structure $(b q(b / 2) q b)$, there is now strong coupling between the two central states and the two edge states. The edge states rapidly move toward the central region. Inserting another two binary unit cells produces the familiar structure $b q b q b$ and the black transmission profile. 
Doubling the central region causes coupling of the states in each pair to weaken due to the increased distance bewteen the interface pairs $b q b$. This is seen in the magenta curve as the four peaks mostly merge into two, recovering Fig. 13(a) at $d_{C}=0.25$. There is also a new pair of edge states in Fig. 14(a).

An important change occurs in the transmission behavior as $d_{C}$ increases if the sandwiching layers are quaternary. In Fig. 13(d), the heterostructure is $q b q$ and a topological state is observed in the upper half of the map; however, it splits into two separate peaks since there are two interfaces. With additional layers, the structure becomes $q b q b q$ and $q b q b q b q$, shown in Fig. 13(e) and Fig. 13(f) respectively. Here the split topological state is surrounded by resonant states that behave similarily to those described in Fig. 14. To help understand why the topological state appears in the $q b \ldots b q$ but not the $b q \ldots q b$ configuration, it is helpful to examine the behavior of the band at small $d_{C}$. Recall that when Eq. 26 produces opposite signs for the isolated quaternary and binary PCs, the number of resonant states in the middle of the PBG must be equal to the number of interfaces in the composite heterostructure. For $b q \ldots q b$, all the states remain separate (i.e. states do not merge). For example, let's consider Fig. 13(c). Since there are 6 interfaces, the 6 states that enter the band gap are the 3 closest pass band states on either side of the gap. Compare this to Fig. 13(f), where only the two closest states on either side of the PBG wander into the gap when $d_{C}$ increases from 0 . The third closest states to the PBG are seen merging and disappearing with other states in adjacent bands on the far left and far right of the map. As $d_{C}$ continues to increase, there are temporarily only 4 states. Therefore in order to have a total of 6 states, the split topological state must appear after the phase transition.

When the two endlayers are different, we get the sequence $b q \ldots b q$. Figs. 13(g)13(i) are $b q, b q b q$, and $b q b q b q$ respectively. Since there are now an equal number 
of binary and quaternary crystals in the structure, there are an odd number of interfaces and reversing the order of the components $(b q \rightarrow q b)$ will not change the transmission. Fig. 13(g) is the familiar single topological state from heterostruture bq. For Fig. 13(h) \& 13(i), the addition of $b q$ layers produces resonant states that behave like those discussed previously.

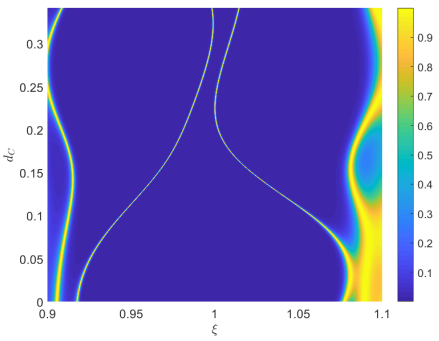

(a)

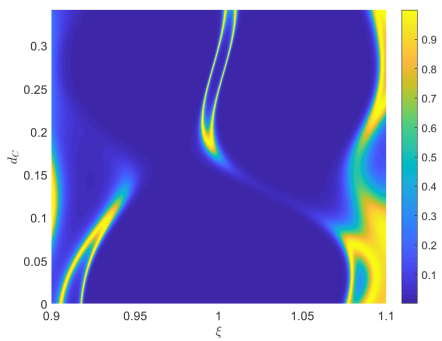

(d)

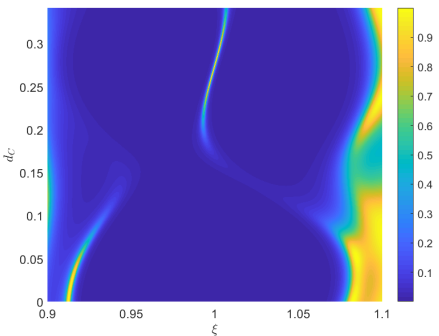

$(\mathrm{g})$

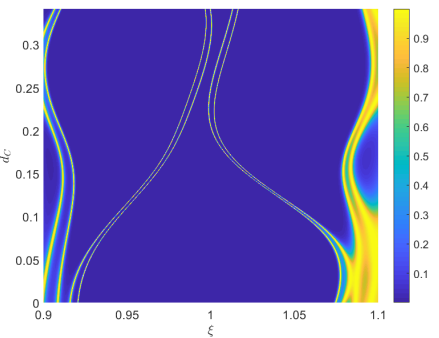

(b)

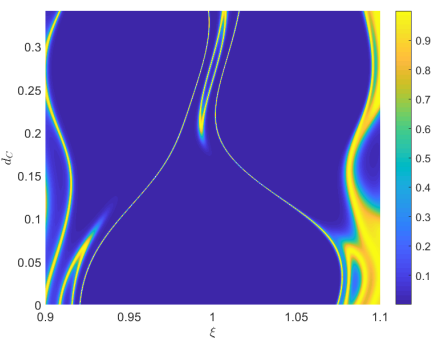

(e)

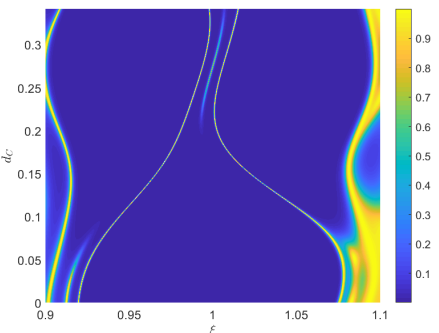

(h)

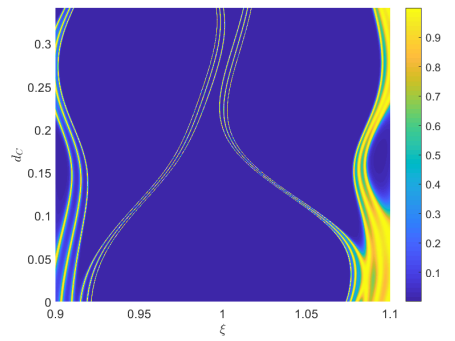

(c)

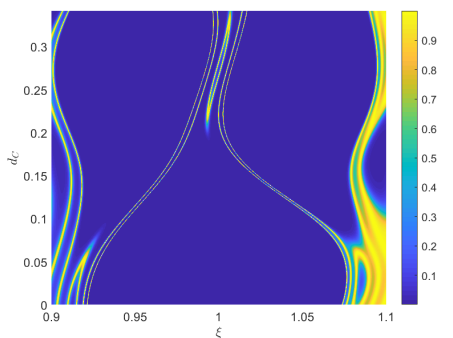

(f)

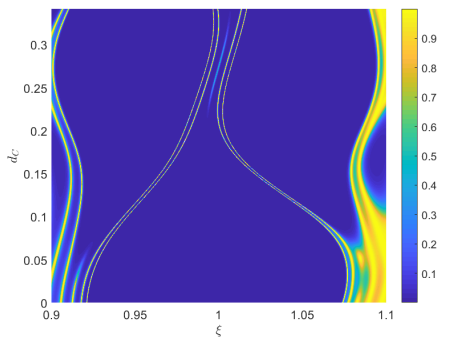

(i)

Figure 13: Transmission map for PC heterostructure in $3^{\text {rd }}$ PBG. The stucture consists of alternating binary $(b)$ and quaternary $(q)$ PCs. Each PC has 4 symmetric unit cells. $\epsilon_{A}=6, \epsilon_{C}=3, \epsilon_{B}=\mu_{A}=\mu_{B}=\mu_{C}=1$ (a) bqb (b) bqbqb (c) $b q b q b q b(\mathrm{~d}) q b q(\mathrm{e}) q b q b q(\mathrm{f}) q b q b q b q(\mathrm{~g}) b q[32]$ (h) $b q b q(\mathrm{i}) b q b q b q$

In our second investigation, layer $C$ is given a permittivity with frequency 


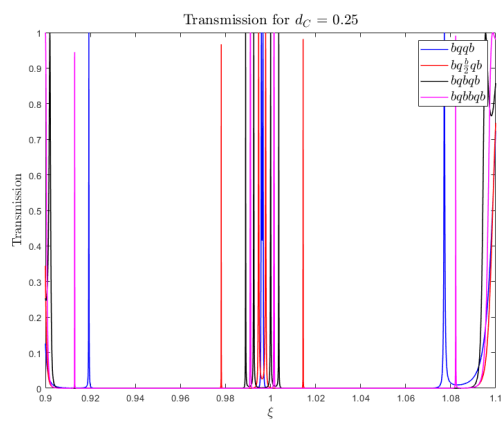

(a)

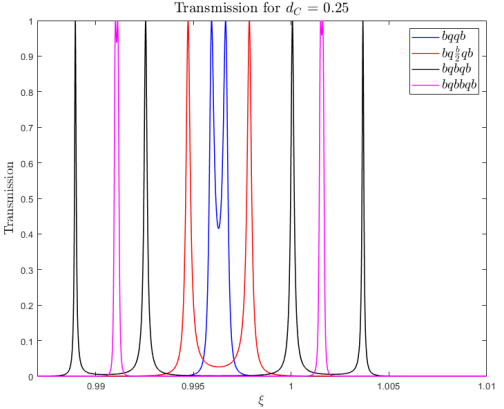

(b)

Figure 14: (a) Transmission resonance behavior for PC heterostucture $b q \ldots q b$ as the number of central binary crystals varies. Each $b$ and $q$ represents 4 unit cells (b/2 is 2 unit cells). (b) Central region of (a).

dependence, in accordance with the Drude model of dispersion,

$$
\epsilon_{C}=1-\frac{\xi_{p}^{2}}{\xi^{2}+i g \xi}
$$

where $\xi_{p}$ and $g$ are the dimensionless plasma and collision frequencies. Eq. 36 is plotted in Fig. 15 with plasma frequency $\xi_{p}=2$ and negligible collision frequency $g=10^{-10}$. Therefore, layer $C$ acts as a metal. Layers $A$ and $B$ remain unchanged. Since the optical path in metal is not constant with frequency, the layer width defined in Eqs. 27 \& 28 are given simplier forms,

$$
\begin{aligned}
& d_{A}=\frac{\gamma-n_{B}}{n_{A}-n_{B}}-d_{C} \\
& d_{B}=\frac{\gamma-n_{A}}{n_{B}-n_{A}}-d_{C}
\end{aligned}
$$

Now, $\gamma$ is only relevant when defining the layer widths before the metal is introduced. As $d_{C}$ increases, the band gap closing points are skewed towards higher frequencies due to the behavior of Eqs. 37 \& 38. As a concequence of this, toppological states in a single interface $b q$ system do not start and terminate at the closing points nor are they positioned near the center of the gap. An example of 
this behavior is shown in Fig. 16. In Fig. 16(a), the transmission map is plotted around $\xi=2$. The metallic layer, $d_{C}$, follows the behavior in Fig. 15. Note that we can have a case where one gap (top center) can support two states. The left state is much sharper than the right one. Also worth noting is that the two center states appear to cross the plasma frequency of the metallic layer without anything unusual happening. This is acceptable because the effective plasma frequency of the entire heterostructure is much lower than the plasma frequency of the metallic inclusion, so the effective permittivity of the heterosructure is positive in the region of these states [35]. This means that all visible gaps in Fig. 16(a) are classifed as PBGs. There are also two distinct groups of Fabrey-Perot resonances. The brighter, more slanted triplets that largely encase the PBGs are caused by coupling among the 3 interfaces of the four unit cells in the quaternary PC. There is also a fainter vertical triplet of resonances between about $1.72<\xi<1.9$, that is caused by the three interfaces in the binary PC. As $d_{C}$ increases, the leftmost topological state eventually appears to turn into one of these resonances and the rightmost of these states breaks away to become the top-center topological state. The equation $\operatorname{Im}\left(Z_{b}+Z_{q}\right)=0$ is plotted in Fig. 16(b), showing the exact location of those five topological states.

As in the all dielectric case, when there are multiple binary/quaternary interfaces, topological states can split; however, the split states are much closer together, meaning that they are more difficult to resolve. Transmission maps for the $q b q$ and $b q b$ configurations are displayed in Fig. 17. While they look very similar to each other and to the single interface system, some subtleties can be pointed out. Resonances in the $q b q$ system are much sharper compared to those in $b q b$. Also the splitting can be seen, although it is more pronounced in $q b q$. Cross sections of the lower center topological state for $d_{C}=0.1$ are shown in both structures 
in Fig. 18 as the number of interface increases. In Fig. 18(a), the transmission is shown for a heterostructures sandwiched between two quaternary PCs. As the number of interfaces increases, each split state itself divides such that the total number equals the number of interfaces. Fig. 18(b) zooms into the left cluster of states. If the heterostructure is bounded by binary PCs, shown in Fig. 18(c), the two central split states appear much closer together. As the number of interfaces increases these two eventually merge and the resultant peak decreases. In the plot, this occurs for six interfaces $(b q b q b q b)$. This makes it appear that there is a missing state; however, similar to the all dielectric heterostructures $q b \ldots b q$ (Figs. 13(d)-13(f)), this merging occurs at lower values of $d_{C}$ as the number of interfaces increases. Therefore if the transmission cross section was taken for, say, $d_{C}=0.08$ rather than for $d_{C}=0.1$, then the central peak for structure $b q b q b q b$ would instead appear as a small doublet, bringing the total number of states to six.

To help understand what is happening within the heterostructure, it is beneficial to compare the optical system to the more familar 1D coupled harmonic oscillator, shown in Fig. 19. The interfaces between the individual PCs act as identical masses and the PCs themselves can be thought of as the spring constants. Since there are two different PCs, two distinct spring constants are used. In this example, the constant $k$ corresponds to the binary PC while $\kappa$ corresponds to the quaternary PC or vice virsa. The topological state will split into a number of states corresponding to the number of interfaces. With an even number of interfaces, the central state vanishes and splits such that half are above the original frequency and half are below. Using this analogy with two interfaces, the lower of the two states is the symmetric state while the higher one is the antisymmetric state [36]. With an odd number of interfaces, the central state still splits as in the 
even case except now the original state remains.This splitting is shown in Fig. 20. Overall, the original and split frequencies can be related by an average,

$$
\xi_{0}^{2}=\frac{1}{N} \sum_{i=1}^{N} \xi_{i}^{2}
$$

where $N$ is the number of PCs in the entire structure and the index, $i$, is summed through all frequencies after the splitting.

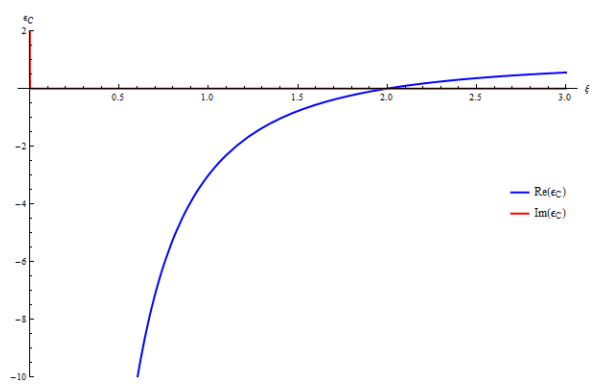

Figure 15: Real and imaginary parts of $\epsilon_{C}$ for metallic layer $C$. Plasma frequency $\xi_{p}=2$ and collision frequency $g=10^{-10}$. Frequencies are scaled according to $\xi=f \Lambda / c_{0}$.

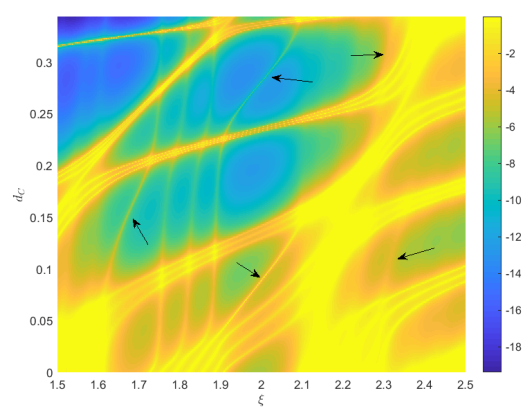

(a)

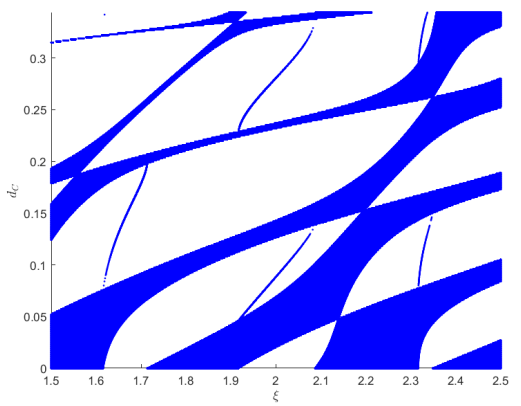

(b)

Figure 16: (a) Transmission map behavior for PC heterostucture $b q$ where the quaternary PC contain metallic layer $C$. $\epsilon_{A}=6, \epsilon_{B}=\mu_{A}=\mu_{B}=\mu_{C}=1$. $\epsilon_{C}$ is given by Eq. 36. Note the five topological states indicated by the arrows. The color scheme is logarithmic. (b) These states correspond to where the imaginary part of the impedance is zero. 


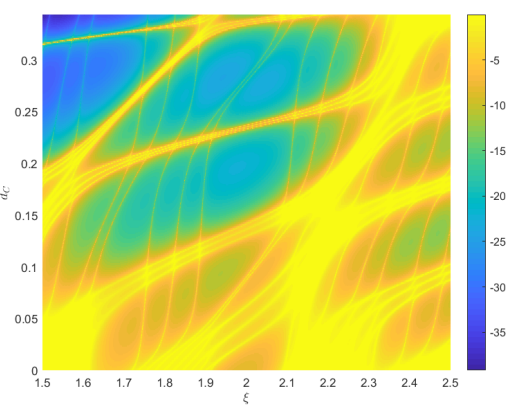

(a)

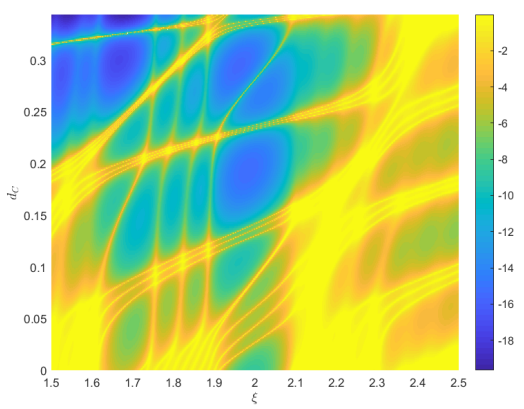

(b)

Figure 17: Transmission map for a double interface heterostructure. Layer $C$ of the quaternary $\mathrm{PC}$ is metallic. Configuration is (a) $q b q(\mathrm{~b}) b q b$

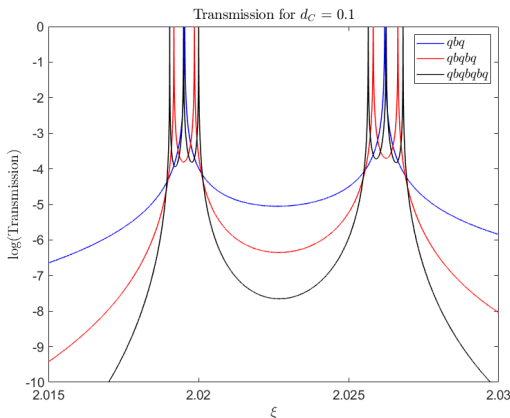

(a)

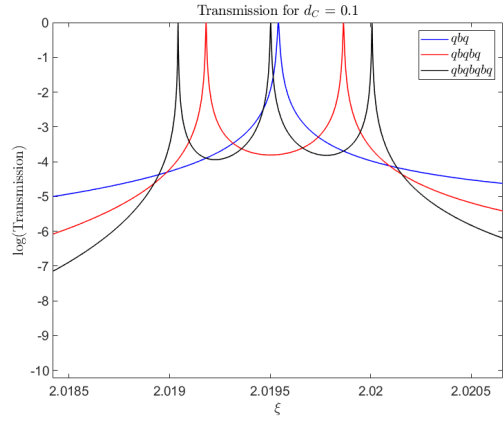

(b)

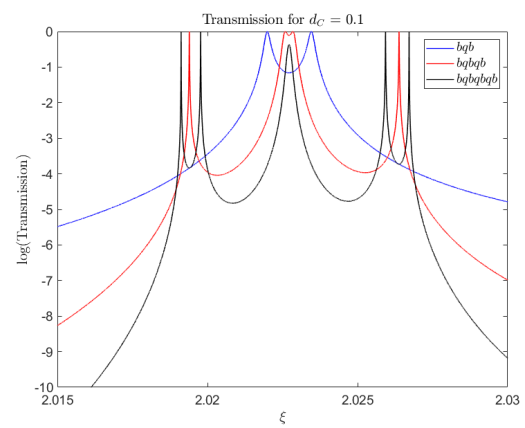

(c)

Figure 18: Transmission cross section for the heterostucture in Fig. 12 with layer $C$ having frequency dependence described by Eq. 36. All other $\epsilon$ and $\mu$ values are the same as in Fig. 13. (a) Transmission for structures of form $q b . . b q$. (b) Zoomed in version of left collection of peaks in (a). (c) Transmission for structures of form $b q \ldots q b$. 


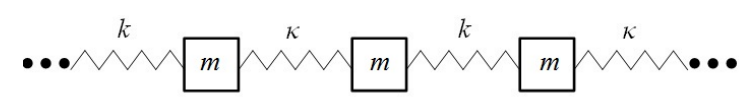

Figure 19: Analogous coupled oscillator model of the heterostructure shown in Fig 12. The spring constants represent the PCs and the masses represent interfaces between PCs.

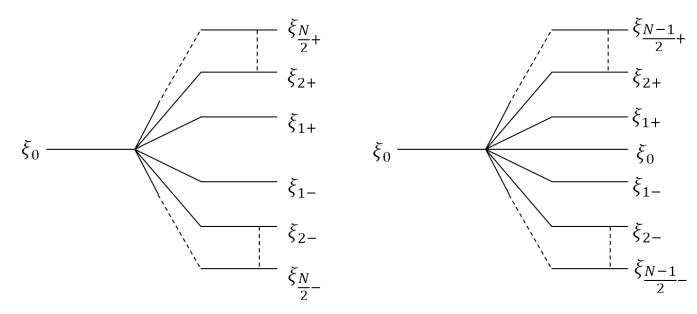

Figure 20: Schematic showing the splitting of the original interface state. The number of interfaces is $N$. The left diagram represents an even number of interfaces while the right one is for an odd number of interfaces.

\subsection{Conclusion}

We have described the evolution of resonant states in a photonic heterostructure composed of alternating binary and quaternary inversion symmetric photonic crystals as the quaternary crystal transforms from one binary to another. This was done by making the tunable layer in the quaternary crystal a free parameter. Two different heterostructures were given, one in which all components were dielectrics and one in which the free parameter was frequency dependent. For the all dielectric case, as shown in Fig. 13, the maximum number of resonant states in a PBG is equal to the number of $\mathrm{PC}$ interfaces for all configurations shown. All configurations (except Figs. 13(d) and 13(g)) possess resonances that started as pass band states for $d_{C}=0$, but the central topological state can vanish, split in two, or remain intact, depending on if the heterostructure is $b q \ldots q b, q b \ldots b q$, or $b q \ldots b q$ respectively. In Fig. 17, it was shown that if the additional layer in the quaternary $\mathrm{PC}$ has frequency dependence, some of the split modes can become much more compressed and difficult to resolve, more so for $b q b$ than $q b q$. Without a constant optical path, the topological states in Figs. 16 and 17 do not start and 
end at PBG closing points, but rather on the edges. These results show that it is possible to generate sequences of resonant states in a binary-quaternary PC heterostructure solely through the manipulation of the geometry of a heterostructure. The fabrication of such a structure could be useful for filtering applications. 


\subsection{References \\ List of References}

[1] E. Yablonovitch, Inhibited Spontaneous Emission in Solid-State Physics and Electronics, Phys. Rev. Lett. 58, 2059 (1987).

[2] S. John, Strong localization of photons in certain disordered dielectric superlattices, Phys. Rev. Lett. 58, 2486 (1987).

[3] E. Istrate, M. Charbonneau-Lefort, and E. H. Sargent, Theory of photonic crystal heterostructure, Phys. Rev. B 66, 075121 (2002).

[4] E. Istrate, and E. H. Sargent, Photonic crystal heterostructures and interfaces, Rev. Mod. Phys. 78, 455 (2006).

[5] I. Tamm, Über eine mögliche Art der Elektronenbindung an Kristalloberflächen, Z. Phys. 76, 849 (1932).

[6] A. P. Vinogradov, A. V. Dorofeenko, S. G. Erokhin, M. Inoue, A. A. Lisyansky, A. M. Merzlikin, and A. B. Granovsky, Surface state peculiarities in one-dimensional photonic crystal interfaces, Phys. Rev. B 74, 045128 (2006).

[7] A. P. Vinogradov, A. V. Dorofeenko, A. M. Merzlikin, and A. A. Lisyansky, Surface states in photonic crystals, Sov. Phys.-Usp 53, 243 (2010).

[8] A. V. Kavokin, I. A. Shelykh, and G. Malpuech, Lossless interface modes at the boundary between two periodic dielectric structures, Phys. Rev. B 72, $233102(2005)$.

[9] D. Gao, W. Mao, R. Zhang, J. Liu, Q. Zhao, W. Y. Tam, and X. Wang, Tunable interface state in one dimensional composite photonic structure Opt. Comm. 453, 124324 (2019)

[10] L. L. Lin, and Z. Y. Li, Interface states in photonic crystal heterostructures, Phys. Rev. B 63, 033310 (2001).

[11] S. Feng, H. Y. Sang, Z. Y. Li, B. Y. Cheng, and D. Z. Zhang, Sensitivity of surface states to the stack sequence of one-dimensional photonic crystals, J. Opt. A: Pure Appl. Opt. 7, 374 (2005)

[12] Y. Zheng, Y. Wang, J. Lou, and P. Xu, Optical Tamm states in photonic structures made of inhomogeneous material, Opt. Comm. 406, 103 (2018)

[13] O. El Abouti, E. H. El Boudouti, Y. El Hassouani, A. Noual, and B. DjafariRouhani, Optical Tamm states in one-dimensional superconducting photonic crystal, Phys. Plasmas 23, 082115 (2016) 
[14] T. B. Wang, C. P. Yin, W. Y. Liang, J. W. Dong, and H. Z. Wang, Electromagnetic surface modes in one-dimensional photonic crystals with dispersive metamaterials, J. Opt. Soc. Am. B 26, 1635 (2009)

[15] J. Barvestani, M. Kalafi, A. Soltani-Vala, and A. Namdar, Backward surface electromagnetic waves in semi-infinite one-dimensional photonic crystals containing left-handed materials, Phys. Rev. A 77, 013805 (2008)

[16] A. Namdar, I. V. Shadrivov, and Y. S. Kivshar, Backward Tamm states in left-handed metamaterials, Appl. Phys. Lett. 89, 114104 (2006)

[17] H. Hajian, B. Rezaei, A. S. Vala, and M. Kalafi, Tuned switching of surface waves by a liquid crystal cap layer in one-dimensional photonic crystals, Appl. Optics 51, 2909 (2012)

[18] J. Bashiri, B. Rezael, J. Barvestani, and C. J. Zapata-Rodriguez, Bloch surface waves engineering in one-dimensional photonic crystals with a chiral cap layer, J. Opt. Soc. Am. B 36, 2106 (2019)

[19] M. Kaliteevski, I. Iorsh, S. Brand, R. A. Abram, J. M. Chamberlain, A. V. Kavokin, and I. A. Shelykh, Tamm plasmon-polaritons: Possible electromagnetic states at the interface of a metal and a dielectric Bragg mirror, Phys. Rev. B 76, 165415 (2007)

[20] S. Brand, M. A. Kaliteevski, and R. A. Abram, Optical Tamm states above the bulk plasma frequency at a Bragg stack/metal interface, Phys. Rev. B 79, 085416 (2009)

[21] H. Zhou, G. Yang, K. Wang, H. Long, and P. Lu, Multiple optical Tamm states at a metal-dielectric mirror interface, Opt. Lett. 35, 4112 (2010)

[22] M. Xiao, Z. Q. Zhang, and C. T. Chan, Surface Impedance and Bulk Band Geometric Phases in One-Dimensional Systems, Phys. Rev. X 4, 021017 (2014)

[23] J. Zak, Berry's Phase for Energy Bands in Solids, Phys. Rev. Lett. 62, 2747 (1989)

[24] K. H. Choi, C. W. Ling, K. F. Lee, Y. H. Tsang, and K. H. Fung, Simultaneous multi-frequency topological edge modes between one-dimensional photonic crystals, Opt. Lett. 41, 1644 (2016)

[25] L. Wang, W. Cai, M. Bie, X. Zhang, and J. Xu, Zak phase and tolopological plasmonic Tamm states in one-dimensional plasmonic crystals, Opt. Express 26, 28963 (2018)

[26] Y. Yang, T. Xu, Y. F. Xu, and Z. H. Hang, Zak phase induced multiband waveguide by two dimensional photonic crystals, Opt. Lett. 42, 3085 (2017) 
[27] Y. Fei, Y. Liu, D. Dong, K. Gao, S. Ren, and Y. Fan, Multiple adjustable optical Tamm states in one-dimensional photonic quasicrystals with predisigned bandgaps, Opt. Express 26, 34872 (2018)

[28] I. Iorsh, P. V. Panicheva, I. A. Slovinskii, and M. A. Kaliteevski, Coupled Tamm Plasmons, Tech. Phys. Lett. 38, 351 (2012)

[29] M. Durach, and A. Rusina, Transforming Fabry-Perot resonaces into a Tamm mode, Phys. Rev. B 86, 235312 (2012)

[30] J. D. Cox, J. Sabarinathan, and M. R. Singh, Resonant Photonic States in Coupled Heterostructure Photonic Crystal Waveguides, Nanoscale Res. Lett. 5, $741(2010)$

[31] J. Hu, W. Liu, W. Xie, W. Zhang, E. Yao, Y. Zhang, and Q. Zhan, Strong coupling of optical interface modes in a $1 D$ topological photonic crystal heterostructure/Ag hybrid system, Opt. Lett. 44, 5642 (2019)

[32] N. Bianchi, and L. Kahn, Topological Photonic States at a 1-D BinaryQuaternary Interface, arXiv:1910.02920v1. (submitted for publication)

[33] A. Yariv, and P. Yeh, Optical Waves in Crystals: Propagation and Control of Laser Radiation, (John Wiley \& Sons, 1984).

[34] S. J. Orfanidis, Electromagnetic Waves and Antennas, (Rutgers University, 2016).

[35] J. Manzanares-Martinez, ANALYTIC EXPRESSION FOR THE EFFECTIVE PLASMA FREQUENCY IN ONE-DIMENSIONAL METALLICDIELECTRIC PHOTONIC CRYSTAL, Progress In Electromagnetic Research M 13, 189 (2010).

[36] S. T. Thornton, and J. B. Marion, Classical Dynamics of Particles and Systems, (Cengage Learning, 2008). 


\title{
MANUSCRIPT 3
}

Branch point crossings and interface states in quaternary photonic crystals using effective medium theory

\author{
Nicholas J Bianchi and Leonard M Kahn \\ University of Rhode Island \\ Physics Department
}

This manuscript is being prepared for submission. 


\subsection{Abstract}

Effective medium theory is used to model a one dimensional lossy dielectric quaternary photonic crystal as a homogeneus slab. The unit cell of the original crystal is inversion symmetric with layer sequence ACBCA. The behavior of the branch frequency singularities in the effective refractive index is investigated as parameters in the layered structure change. A heterostructure composed of multiple photonic crystals is also modeled with effective medium theory. It is shown that the effective refractive index of such a structure possesses regions of normal dispersion that corresponding to localized interface states within the overall regions of anaomalous dispersion corresponding to the photonic band gap.

\section{$3.2 \quad$ Introduction}

Effective medium theory (EMT) is the modeling of scattering parameters, such as reflection and transmission, from a complex heterogeneous structure by replacing it with a partially or fully homogenized material, in an effort to simplify a problem. While any field of wave mechanics can make use of EMT, it has seen significant applications in electromagnetism (EM). In 1956, Rytov [1] proposed that a one dimensional Bragg grating composed of two different isotropic layers could be thought of as a uniform slab with anisotropic effective permittivity and permeability. Since then, EMT has been used to model both photonic crystals $[2,3]$, which have scattering elements on the order of the incident wavelength, and metamaterials [4], where wavelength is several orders of magnitude larger. In the long wavelength regime, EMT was applied to $2 \mathrm{D}[5,6,7]$ and $3 \mathrm{D}[8,9]$ periodic photonic crystals (PCs) by approximating them as 1D Bragg gratings. Reflection from a 1D Bragg grating at oblique angles using EMT was examined [10, 11].

EMT has also been applied the photonic systems for higher frequencies. In 1976, Yariv and Yeh [12] used an effective index profile representation of a binary 
PC whose layers have the same optical path to achieve nonlinear phase matching. It was shown that when a PC is homogenized, the resultant slab behaves as a single negative material inside the photonic band gaps (PBGs) [13]. Later, it was found that a bi-anisotropic parameter (magnetoelectric coupling) is present when homogenizing unit cells without inversion symmetry $[14,15]$. These works also showed that power expansions in frequency for effective parameters becomes invalid at the start of the first PBG. This breakdown was due to the presence of branch points (i.e. singularites) [14]. Liu [14] also showed that effective index and impedance can be modeled with the Kramers-Kroing relations. Various concepts, such as density of states [16], reflection phase [17, 18], and defects [19] have been studied in PCs using EMT.

In this work, we base the design of the laminated PCs from Ref. [20]. The individual layers in the symmetric unit cell are denoted as $A, B$, and $C$ with refractive indices $n_{A}, n_{B}$, and $n_{C}$. The permittivity, $\epsilon$ and permeability, $\mu$ as defined as $n_{i}^{2}=\epsilon_{i} \mu_{i}$ The period, $\Lambda$ and optical path, $\Gamma$, across a unit cell are given as constants, where $\gamma=\Gamma / \Lambda$. As a result, the scaled layer lengths are,

$$
\begin{aligned}
& d_{A}=\operatorname{Re} \frac{\gamma-n_{B}-2\left(n_{C}-n_{B}\right) d_{C}}{n_{A}-n_{B}} \\
& d_{B}=\operatorname{Re} \frac{\gamma-n_{A}-2\left(n_{C}-n_{A}\right) d_{C}}{n_{B}-n_{A}}
\end{aligned}
$$

From the given $\epsilon$ and $\mu$ in the PC, the transfer matrix method [21, 22] is used to calculate transmission and reflection spectra. Then, applying EMT, these spectral functions are used to recover effective index and impedance relations in the associated uniform slab. The primary purpose of this work is to examine the behavior of branch intersections in the effective refractive index as parameters in the corresponding laminated PC vary. It is a well known problem that recovering 
an effective refraction index from transmssion and reflection functions introduces extraneous solutions in the form of multiple branches since the cosine function is multi-valued [23]. The goals of this work are to: (1) review the methods of effective medium theory for one-dimensional systems and compare the analytic and numerical techniques for finding effective parameters, (2) apply EMT to quaternary PCs with tunable parameters to show how branch intersections evolve as said parameters change, and (3) describe a heterostructure composed of binary and quaternary PCs in terms of EMT and explain interface modes in such a system in terms of the effective parameters.

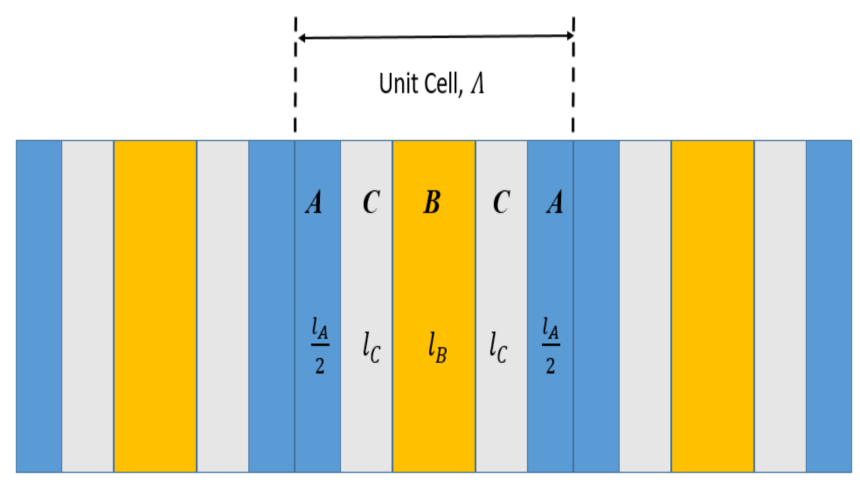

(a)

Figure 21: Inversion symmetric quaternary photonic crystal

\subsection{Methods}

While in most scattering problems the objective is to find reflection and transmission spectra based on the given material parameters (i.e. $\epsilon \& \mu$ or $n \& z)$, with effective medium theory the inverse problem can be performed. In this case, a system has a known or measured spectrum. With this information, a more uniform object can be considered that produces the same scattering. Essentially, this means that for the uniform material to return the same scattering information as the heterogeneous one, the object must act as though it has frequency dependent dispersion rather than spatial dispersion. 
Smith et. al[23] provided an analytical method for extracting the effective refractive index and impedance from the transmission and reflection coefficents, $t$ and $r$ respectively. Matching electric and magnetic boundary conditions for a single slab yields,

$$
\begin{gathered}
t^{-1}=\cos \phi-\frac{i}{2}\left(z+\frac{1}{z}\right) \sin \phi \\
r t^{-1}=-\frac{i}{2}\left(z-\frac{1}{z}\right) \sin \phi
\end{gathered}
$$

where $\phi=n_{e f f} k_{0} L$ for freespace wavevector, $k_{0}=\omega / c$, and slab length, $L$. Using dimensionless variables, $\phi=2 \pi n_{e f f} \xi N$. The length must be an integer multiple, $N$, of the unit cell period, $\Lambda$, and $\xi=f \Lambda / c$ is the scaled frequency. Equations 42 and 43 can then be inverted to produce,

$$
\begin{gathered}
z^{2}=\frac{(r+1)^{2}-t^{2}}{(r-1)^{2}-t^{2}} \\
\cos \phi=X(\xi)=\frac{1}{2 t}\left(1+t^{2}-r^{2}\right)
\end{gathered}
$$

Finding the permittivity and permeability is now trivial, $\epsilon=n / z$ and $\mu=n z$.

It is assumed that the dielectrics in the original unit cell can only have lossy components. Therefore, the positive root is chosen for the impedance in Eq. 44, since the materials are passive [23]. While symmetric unit cells produce well defined, unique values of impedance, it was found that when homogenizing an asymmetric cell, impedance will depend on the order of the layers. This discrepancy is due to the diagonal scattering matrix elements, $S_{11}$ and $S_{22}$ having different amounts of phase advance [24]. Calculating the effective refractive index is more difficult due to the presence of the cosine function. Mathematically, there are inifinite values of $n_{\text {eff }}$ that satisfy Eq. 45, but, physically, there should only be one. 
As before, to avoid phase ambiguity, it is best to consider symmetric unit cells, although $n_{\text {eff }}$ dependence on unit cell asymmetry is less pronounced compared with $z_{\text {eff }}[24]$. Since the original system is periodic, the thickness of the slab should be taken as small as possible to minimize the number of roots in $\cos \phi$; however, since $L=N \Lambda$, the smallest value of $L$ is a single unit cell $(N=1)[23,24,25]$.

If we let $n_{\text {eff }}=n_{R}+i n_{I}$ and $X=X_{R}+i X_{I}$, Eq. 45 can be separated into $[23]$

$$
n_{R}(\xi)=\frac{1}{2 \pi N \xi}\left[\operatorname{Re}\left(\cos ^{-1}(X(\xi))\right)+2 \pi m\right]
$$

and

$$
n_{I}(\xi)=\frac{1}{2 \pi N \xi} \operatorname{Im}\left(\cos ^{-1}(X(\xi))\right)
$$

where $m$ is an integer that denotes the current branch. Figure 22 shows an example of the effective parameters for a photonic crystal with inversion-symmetric quaternary unit cells. Only 1 unit cell is used. Figure 22a shows the effective impedance using Eq. 44. In Fig. 22b, which displays Eq. 46, branch crossing points, denoted by black dots, only occur in regions of anomalous dispersion, where $\frac{d n_{I}}{d \xi}<0$. Figure 22c displays the real part but without the extra branches. Some early attempts at plotting $\operatorname{Re}\left(n_{\text {eff }}\right)$ have resulted in erroneous solutions, such as incorrect branch transitions [26], and incorrect or missing anomalous behavior [27, 28]. The corresponding crossing points are shown in the imaginary part in Fig. 22d. The positions of these points can be found by setting $X_{I}(\xi)=0$ and are independent of $N$. This equation will be used in the next section to explore a rich supply of behaviors of the branch crossing points. Increasing $N$, however, would cause additional crossing points to appear in the regions of normal dispersion, where $\frac{d n_{R}}{d \xi}>0$, with the number of crossing points in these region equal to $N-1$.

An alternative method to find $n_{R}$ and $n_{I}$ is to calculate them numerically. 


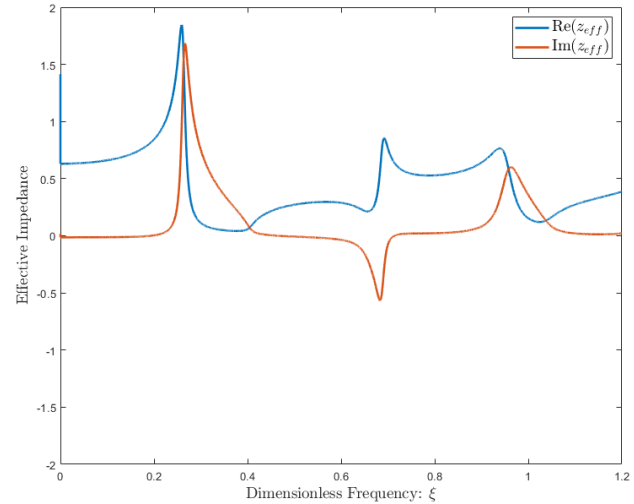

(a)

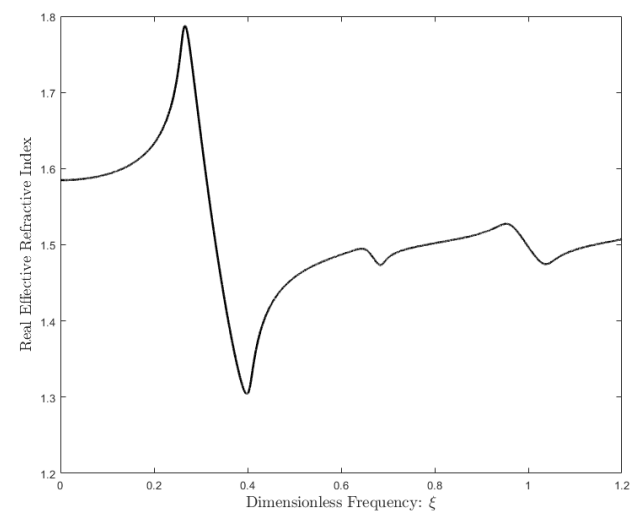

(c)

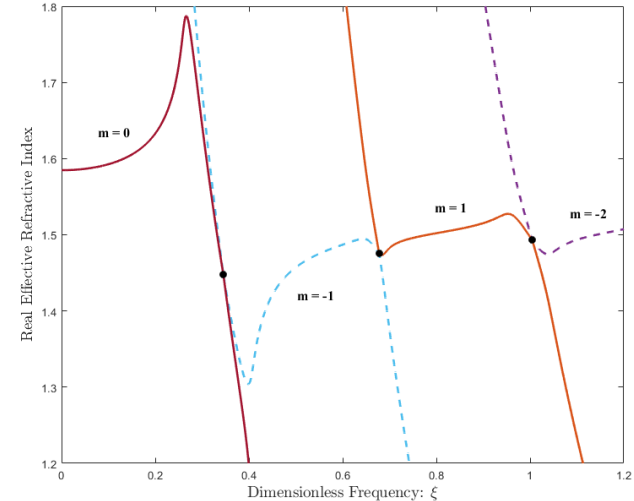

(b)

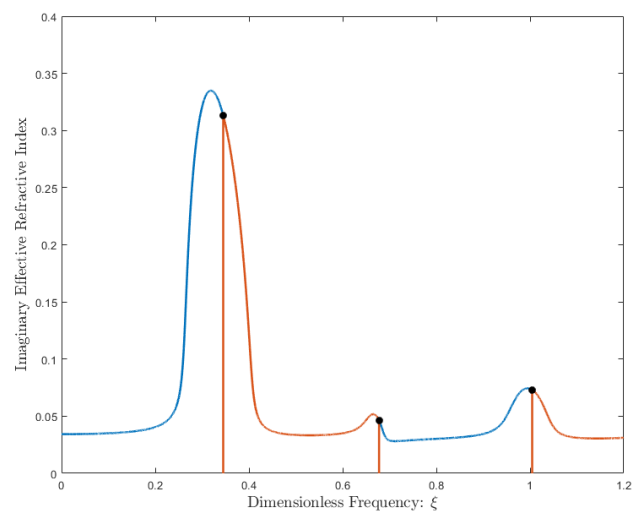

(d)

Figure 22: Example of effective parameters of 1 unit cell from Fig. 21a. In the original unit cell $d_{A}=0.14207, d_{B}=0.45793, d_{C}=0.2, \epsilon_{A}=6+0.2 i, \mu_{A}=1$, $\epsilon_{B}=1, \mu_{B}=1, \epsilon_{C}=3+0.2 i$, and $\mu_{C}=1$.(a) Real and imaginary effective impedance. (b) Real component of refractive index. The values of $m$ specify the branches to ensure $n_{R}$ is continuous and smooth. The black dots highlight the points where the branches connect. (c) Real component of refractive index after nonphysical solutions are removed. (d) Imaginary component of refractive index.

Starting from Eq. 45, we can separate out the real and imaginary equations [21],

$$
\begin{aligned}
& \cos \left(2 \pi N \xi n_{R}\right) \cosh \left(2 \pi N \xi n_{I}\right)=X_{R}(\xi) \\
& \sin \left(2 \pi N \xi n_{R}\right) \sinh \left(2 \pi N \xi n_{I}\right)=-X_{I}(\xi)
\end{aligned}
$$

Note that these equations are both coupled and nonlinear, making solutions challenging to find. As before, the imaginary component is easier to obtain since it is 
only located in hyperbolic functions, meaning that there is only a finite number of possible values it can have; however, we see again that the real component only appears in circular trigonometric functions, meaning we must be careful to select the correct branch. To proceed, it is best to start at zero frequency or at least very close to it. Here all non-physical solutions will diverge. The frequency $\xi$ is run through a list of values and both components are calculated at each step, gradually building an array of values. The main difficulty is selecting an appropriate initial starting value each interation so that $\left(n_{R}, n_{I}\right)$ converges to the correct solution. While the numerical method can still be used at higher frequencies (or higher $N$ ), its practicality starts to falter as possible solutions become closer and closer together, making selecting the correct one more of a challenge. Figure 23 illustrates this problem by logarithmically plotting the difference between the analytical and numerical transmission spectra for 1 and 3 uinits cells. Crystal parameters are the same as in Fig. 22. In Fig. 23a, a single unit cell is used, allowing the arguments of the sine and cosine funtions in Eqs. 48 and 49 to remain small enough to allow for proper convergence of $n_{R}$ and $n_{I}$ for all frequencies. In Fig. 23b, three unit cells are used, and it can be clearly seen that there are three regions, marked by arrows, in which agreement is poor. This is a result of Eqs. 48 and 49 converging to an incorrect solution for those frequency ranges. To remedy this problem for higher $\xi$ (or $N$ ), the same initial $\left(n_{R}, n_{I}\right)$ should not be used throughout the simulation. If it appears that $\left(n_{R}, n_{I}\right)$ will converge to non-physical values for a certain frequency range, then the initial point can be altered for that range.

\subsection{Results and Discussions}

The question now is, how do the crossing frequencies in Fig. 22b evolve as different parameters in the quaternary PC change? As mentioned in the previous section, constructing the real part of the effective refractive index requires knowing 


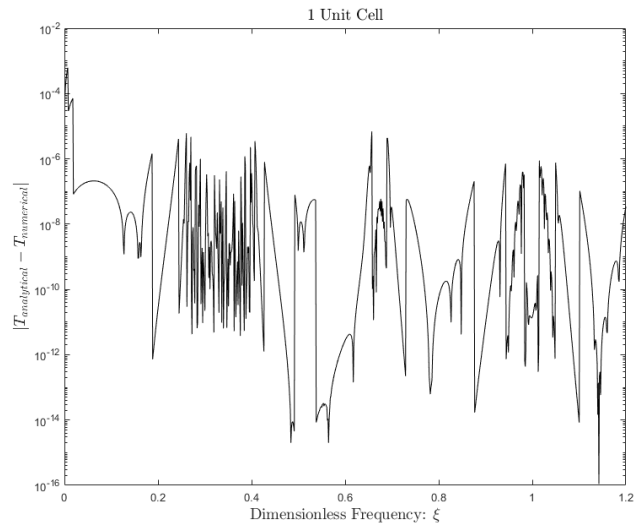

(a)

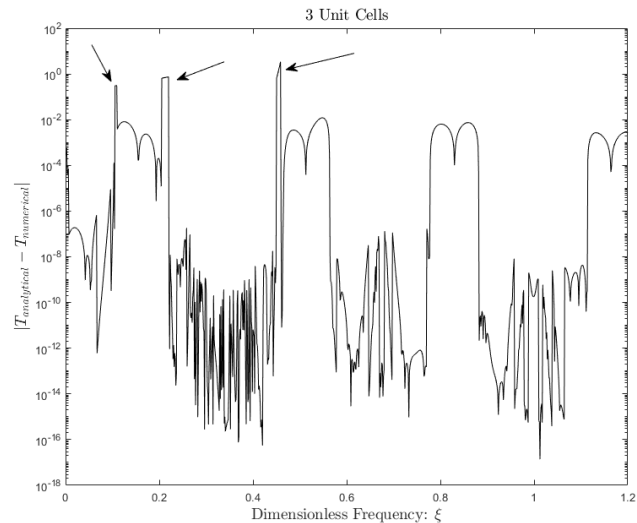

(b)

Figure 23: Difference in transmission calculated analytically with Eqs. 46 and 47 and numerically with Eqs. 48 and 49 (a) 1 unit cell (b) 3 unit cells. Note the regions given by the arrows where convergence is poor. With 3 unit cells the band gaps are more visible, showing better agreement than surrounding areas.

the appropriate branches such that the index is both continuous and smooth. By understanding how properties such as material loss can influence the location of these crossings, the construction of effective index functions can be improved, while reducing the possibility of connecting adjacent branches incorrectly.

We consider a single inversion symmetric quaternary PC made of three different dielectrics, as in Fig. 21. The optical path to unit cell period ratio is $\gamma=\Gamma / \Lambda=1.5$. Only one unit cell is used so that the only crossing points occur in the band gaps. We will consider three different systems and examine the lowest three crossings in each case.

In the first study, $\operatorname{Im}\left(\epsilon_{A}\right)$ was allowed to change, keeping all other parameters constant. The remaining parameters used are: $\operatorname{Re}\left(\epsilon_{A}\right)=6, \mu_{A}=1, \epsilon_{B}=1$, $\mu_{B}=1, \epsilon_{C}=3, \mu_{C}=1$. The results are displayed in Fig. 24. In each plot, the behavior is displayed for four different values of $d_{C}:\{0,0.1,0.2,0.3\}$. For each particular value of $d_{C}$, the general trend is for the crossing frequency to start at a lower value for no loss. Then, as $\operatorname{Im}\left(\epsilon_{A}\right)$ increases, the frequency increases up 


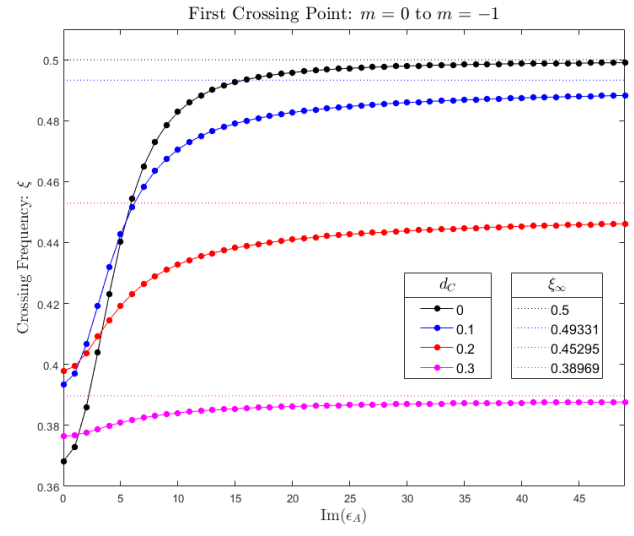

(a)

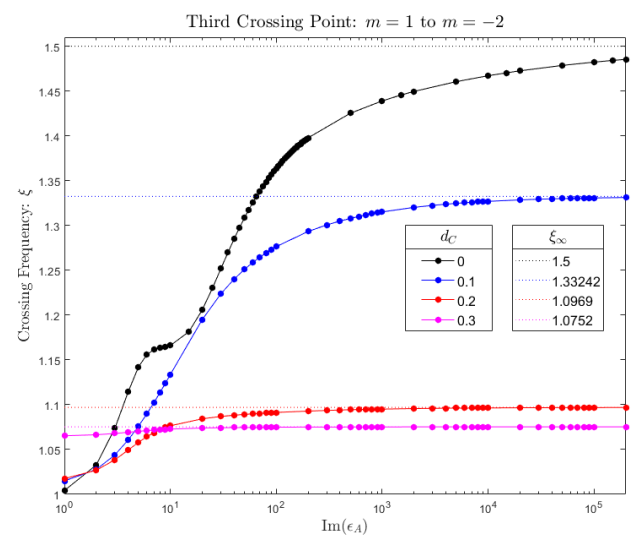

(c)

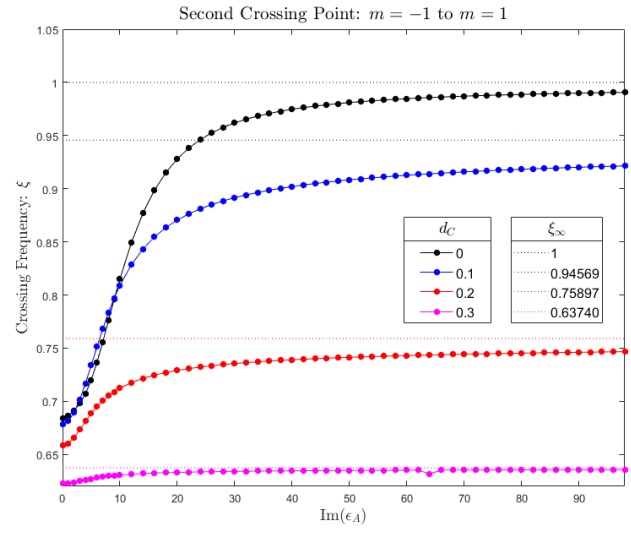

(b)

Figure 24: Behavior of (a) $1^{\text {st }}$ (b) $2^{\text {nd }}$ (c) $3^{\text {rd }}$ crossing point as $\operatorname{Im}\left(\epsilon_{A}\right)$ changes

to some saturation level, $\xi_{\infty}$, marked by horizontal dashed lines. This is because as $\operatorname{Im}\left(\epsilon_{A}\right)$ grows without bound, $d_{A} \rightarrow 0$, in accordance with Eq. 40. In all three figures, as $d_{C}$ increases, there is general trend not only for the saturation frequency to be lower, but also for the initial starting value to be closer to it. This makes sense because as $d_{C}$ increases, $d_{A}$ will go to zero, for the chosen $n_{A}, n_{B}$, and $n_{C}$. The smaller $d_{A}$ is, the less of an impact its loss component will have on the crossing frequnecy. The rate of convergence appears faster in lower band gaps. Both Figs. 24a and 24b show this as the horizontal axis is linear; however, for Fig. 24c, plotting is done on a logarithmic scale to account for the slower 


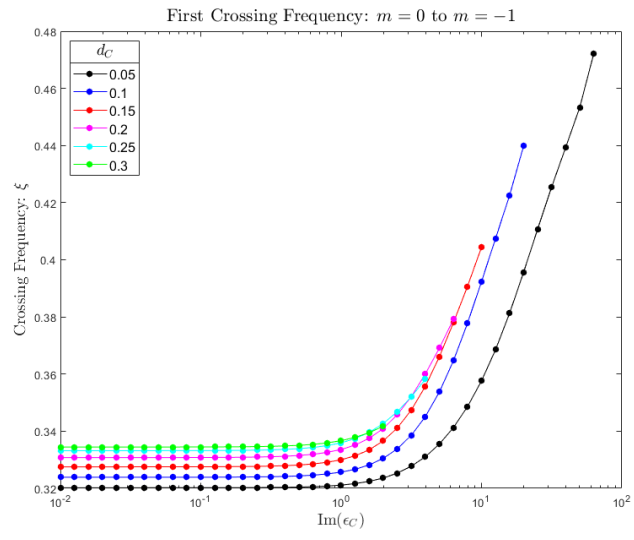

(a)

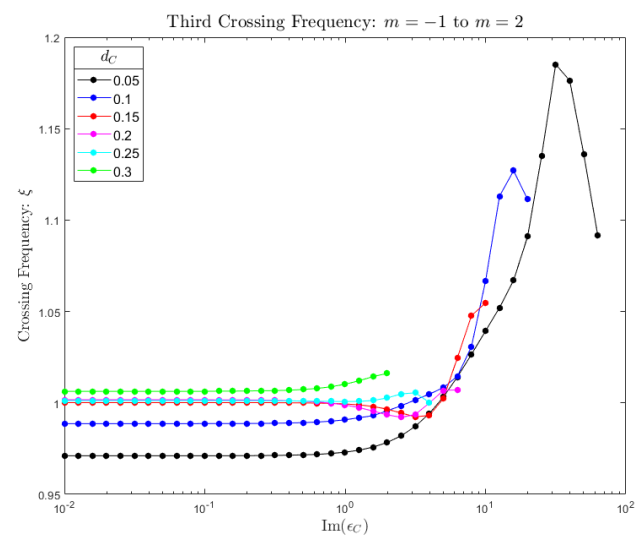

(c)

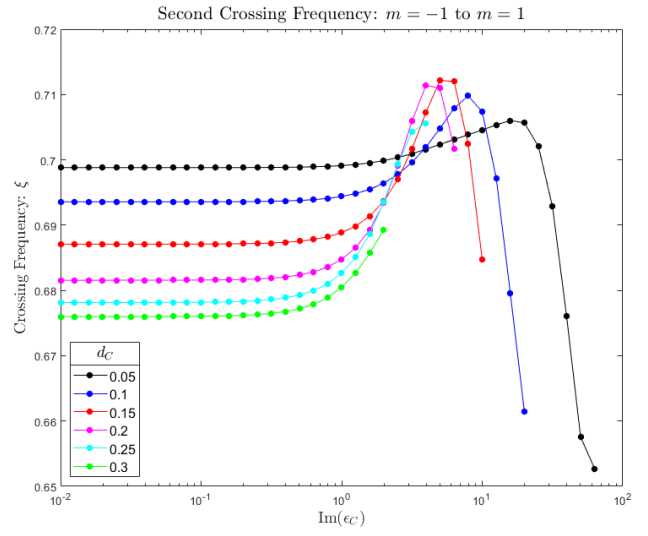

(b)

Figure 25: Behavior of (a) $1^{\text {st }}$ (b) $2^{\text {nd }}$ (c) $3^{\text {rd }}$ crossing point as $\operatorname{Im}\left(\epsilon_{C}\right)$ changes

convergence, especially when $d_{C}=0$. Also in Fig. 24c, note that when $d_{C}=0$, the rate of increase temporarily slows around $\operatorname{Im}\left(\epsilon_{A}\right)=10$ before increasing again.

In the second study, $\operatorname{Im}\left(\epsilon_{C}\right)$ is allowed to change, keeping all other parameters constant. All other parameters are unchanged from the first study and $\epsilon_{A}$ is now lossless. Results are shown in Fig. 25 for six different values of $d_{C}$. Crossing frequencies asymptotically approach constants as $\operatorname{Im}\left(\epsilon_{C}\right) \rightarrow 0$. Note that for larger $d_{C}$, the graphs abruptly end. This is due to the behavior of the layer thicknesses of the corresponding unit cell, given by Eqs. 40 and 41. As $d_{C}$ becomes larger, $d_{A}$ becomes negative and thus invalid at smaller values of $\operatorname{Im}\left(\epsilon_{C}\right)$. The behavior in 


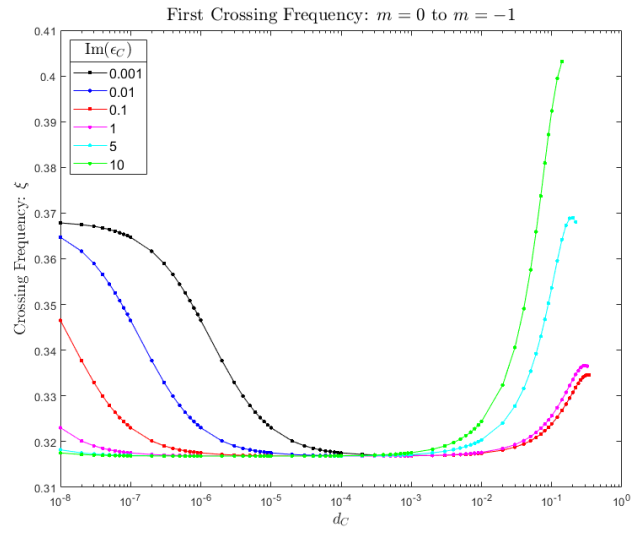

(a)

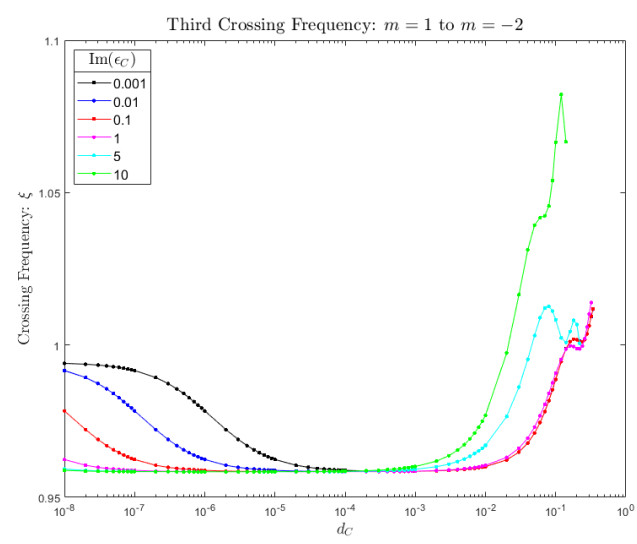

(c)

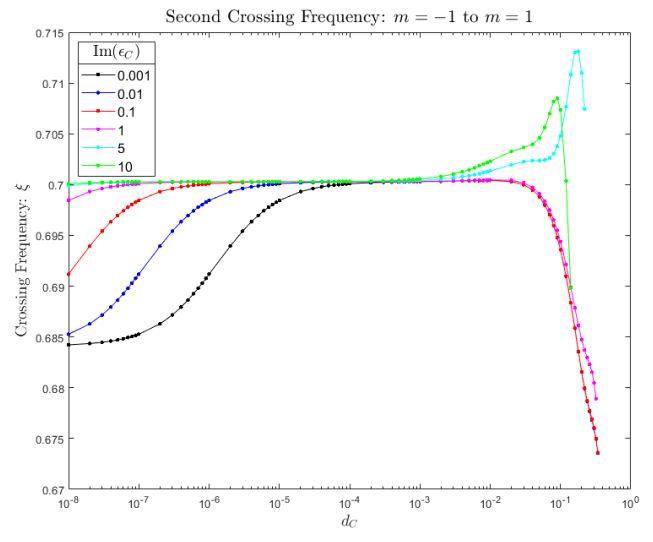

(b)

Figure 26: Behavior of (a) $1^{\text {st }}$ (b) $2^{\text {nd }}$ (c) $3^{\text {rd }}$ crossing point as $d_{C}$ changes

Fig. 25a shows the frequency values increasing monotonically whereas in Fig. 25b and Fig. 25c the frequencies are shown to reach a maximum, at least for smaller $d_{C}$, before sharply declining.

In the third study, $d_{C}$ is allowed to change. Other parameters are: $\epsilon_{A}=$ $6+10^{-8} i, \mu_{A}=1, \epsilon_{B}=1, \mu_{B}=1, \operatorname{Re}\left(\epsilon_{C}\right)=3, \mu_{C}=1$. The tiny imaginary part of $\epsilon_{A}$ produces noticeable behavior in the crossing frequency values for $d_{C} \sim 10^{-7}$ and below. If $\operatorname{Im}\left(\epsilon_{A}\right)$ were zero, then all values would asymptotically approach 0.31682 in Fig. 26a, 0.70029 in Fig. 26b, and 0.95837 in Fig. 26c; however, nonzero $\operatorname{Im}\left(\epsilon_{A}\right)$ elevates the crossing point values in odd band gaps and depresses 
them in even gaps as $d_{C} \rightarrow 0$. Common behavior among all three plots are that as $\operatorname{Im}\left(\epsilon_{C}\right)$ becomes larger, the crossing frequency terminates at lower $d_{C}$. This is only significant for $\operatorname{Im}\left(\epsilon_{C}\right) \geq 1$ and, as in the second study, is due to Eq. 40 eventually becoming negative, which is unphysical. For larger $d_{C}$, behavior of first, second, and third crossing frequencies is quite different. In Fig. 26a, the frequency rises relatively rapidly, more so for larger $\operatorname{Im}\left(\epsilon_{C}\right)$, before leveling off as $d_{C}$ reaches its maximum value. In Fig. 26b, the frequency achieves a maximum before sharply falling. The maximum for $\operatorname{Im}\left(\epsilon_{C}\right)<1$ is present but much smaller. In Fig. 26c, the crossing point frequencies undergo undulatory behavior.

We now consider a heterostructure consisting of a quaternary PC sandwiched between two identical binary PCs (Fig. 21a but with no $C$ layer) [29]. All crystals in the structure are inversion symmetric and so is the entire structure. A system with a binary PC surrounding by two quaternary PCs would also be valid; however, a system with an equal number of both cystals would not (e.g.quaternary-binary). This is because such system, as a whole is not inversion symmetric depsite the crystals composing it to being so. We can apply the techniques in Section 3.3 to this system and find the overall effective refractive index just as for an individual PC. Now though, we do not have the liberty of setting the effective slab thickness equal to a unit cell since now periodicity is broken. We must set the slab thickness equal to the total length of the heterostructure. Since both crystals have the same unit cell period, $L=(2 m+1) N \Lambda$, where $m$ is an integer. The effective index profile is similar to that described in Ref. [12], except now spatially dispersive behavior, caused by the breaking of periodicity via the three different PCs, is superimposed onto the overall spatial dispersion described previously. In Ref. [12], material dispersion was superimposesd onto spatial dispersion.

Coupling between the two PC interfaces results in two distinct interface states, 


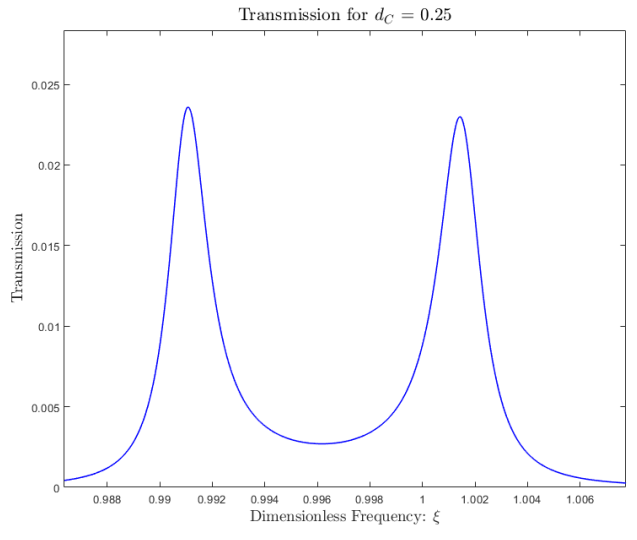

(a)

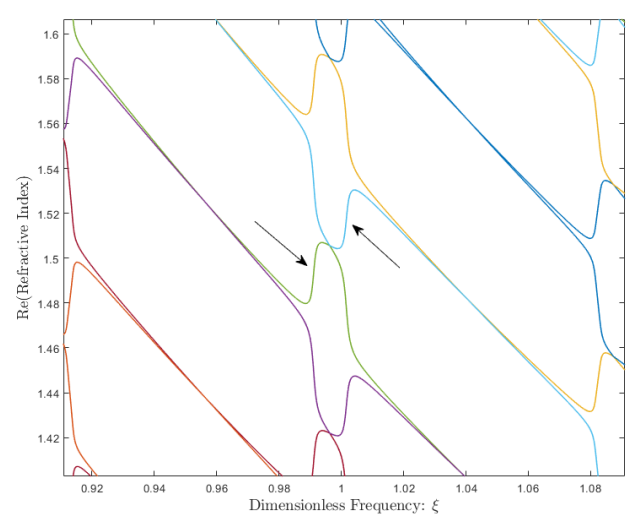

(c)

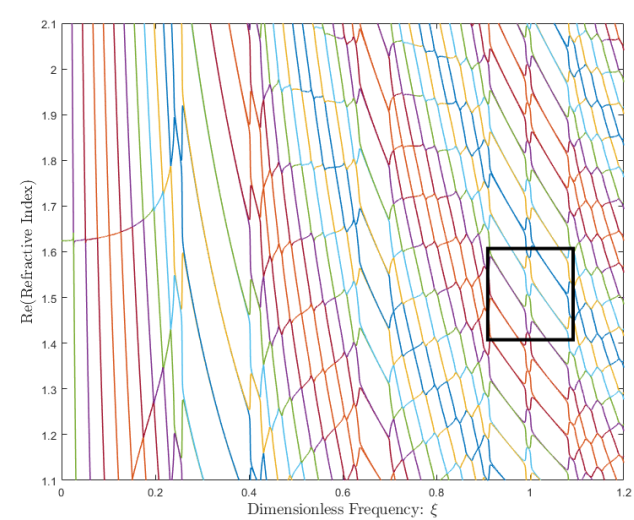

(b)

Figure 27: (a) Two transmission peaks in $3^{\text {rd }}$ band gap for heterostructure: binary/quaternary/binary. Parameters are: $\gamma=1.5, \epsilon_{A}=6+0.01 i, \mu_{A}=1, \epsilon_{B}=1$, $\mu_{B}=1, \epsilon_{C}=3+0.01 i, \mu_{C}=1, d_{C}=0.25 . d_{A}$ and $d_{B}$ are given by Eqs. $40 \&$ 41. (b) Real part of effective refractive index. The boxed region is in (c). (c) The arrows indicate the regions that correspond to the transmission peaks. From left to right, the physcial solution smoothly transitions across the following branches: purple $\rightarrow$ green $\rightarrow$ cyan $\rightarrow$ yellow

displayed in Fig. 27a, in the $3^{\text {rd }}$ band gap for the given parameters: $\gamma=1.5$, $\epsilon_{A}=6+0.01 i, \mu_{A}=1, \epsilon_{B}=1, \mu_{B}=1, \epsilon_{C}=3+0.01 i, \mu_{C}=1, d_{C}=0.25$. If EMT is used to homogenize the entire heterostucture into a uniform slab, the resultant $\operatorname{Re}\left(n_{e f f}\right)$ is displayed in Fig. 27b. The boxed region is displayed in Fig. 27c. Normally, the band gap is represented with a negative derivative in $\operatorname{Re}\left(n_{e f f}\right)$. 
Note, however, the two regions marked by arrows that show brief instances of normal dispersion. These parts correspond to the two transmssion peaks. Small loss terms were added to some of the permittivities to make the branches smooth across the transition points. As in the case for an isolated crystal, the band gaps are represented by a large decrease in the index; however, now each branch crossing contains a small resonance, since the heterostructure, as a whole, is not periodic. This break in periodicity also means the exact behavior of the index is now dependent on the number of unit cells in each PC. As mentioned above, this break in periodicity forces us to use the full length of the structure in Eqs. 46 and 47, leading to a higher number density of branches. It is still possible to apply to analytical and numerical techniques disccssed in Section 3.3 to this system, but it may be more tedious.

\subsection{Conclusion}

We have used effective medium theory to describe some properites of quaternary photonic crystals, both isolated and within heterostructures. For an isolated quaternary PC, the behavior of branch crossing points within the anomalous dispersion region was discussed as various parameters changed. As $\operatorname{Im}\left(\epsilon_{A}\right)$ increases, crossing points eventually reach a saturation frequency, that becomes lower for larger $d_{C}$. In the case of varying $\operatorname{Im}\left(\epsilon_{C}\right)$, keeping all else constant, the crossing points are constant for small $\operatorname{Im}\left(\epsilon_{C}\right)$ but display different behavior depending on the point in question. The highest defined crossing point becomes smaller as $d_{C}$ increases. The roles of $\operatorname{Im}\left(\epsilon_{C}\right)$ and $d_{C}$ are then reversed with $d_{C}$ now as the independent parameter. It is found that asymptotic behavior as $d_{C} \rightarrow 0$ is strongly dependent on $\operatorname{Im}\left(\epsilon_{A}\right)$. The effective index is then examined for a binary/quaternary/binary heterostructure. It was found that interface states within the band gap are represented as rapid increases in an otherwise decreasing region 
of the real part of the refractive index. 


\subsection{References \\ List of References}

[1] S. M. Rytov, Electromagnetic Properties of a Finely Stratified Medium, Sov. Phys. JETP 2, 466 (1956).

[2] E. Yablonovitch, Inhibited Spontaneous Emission in Solid-State Physics and Electronics, Phys. Rev. Lett. 58, 2059 (1987).

[3] S. John, Strong localization of photons in certain disordered dielectric superlattices, Phys. Rev. Lett. 58, 2486 (1987).

[4] D. R. Smith, W. J. Padilla, D. C. Vier, S. C. Nemat-Nasser, and S. Schultz, Composite Medium with Simultaneously Negative Permeability and Permittivity, Phys. Rev. Lett. 76, 4773 (1996)

[5] P. Lalanne, Effective medium theory applied to photonic crystals composed of cubic or square cylinders, Appl. Opt. 35, 5369 (1996)

[6] H. Kikuta, Y. Ohira, H. Kubo, and K. Iwata, Effective medium theory of twodimensional subwavelength gratings in the non-quasi-static limit, J. Opt. Soc. Am. A 15, 1577 (1998)

[7] R. L. Chern, and Y. T. Chen, Effective parameters for photonic crystals with large dielectric contrast, Phys. Rev. B 80, 075118 (2009)

[8] Y. Ono, Transmittance analysis of three dimensional photonic crystals by the effective theory, Appl. Opt. 45, 131 (2006)

[9] S. Datta, C. T. Chan, K. M. Ho, and C. M. Soukoulis, Effective dielectric constant of periodic composite structures, Phys. Rev. B 48, 14936 (1993)

[10] S. Tang, B. Zhu, M. Jia, Q. He, S. Sun, Y. Mei, L. Zhou, Effective-medium theory for one-dimensional gratings, Phys. Rev. B 91, 174201 (2015)

[11] S. Kameda, Effective Medium Theory for Calculating Reflectance from MetalDielectric Multilayered Structure, Jpn. J. Appl. Phys. 51, 042202 (2012)

[12] A. Yariv, and P. Yeh, Electromagnetic propagation in periodic stratified media. II. Birefringence, phase matching, and x-rays lasers, J. Opt. Soc. Am. 67, 438 (1977)

[13] G. Ji-Yong, C. Hong, L. Hong-Qiang, and Z. Ye-Wen, Effective permittivity and permeability of one dimensional dielectric photonic crystal within a band gap, Chinese Phys. 17, 2544 (2008)

[14] Y. Liu, S. Guenneau, and B. Gralak, Causality and passivity properties of effective parameters of electromagnetic multilayered structures, Phys. Rev. B 88, 165104 (2013) 
[15] Y. Liu, S. Guenneau, and B. Gralak, Artificial dispersion via high-order homogenization: magnetoelectric coupling and magnetism from dielectric layers, Proc. R. Soc. London. Ser. A 469, 20130240 (2013)

[16] G. Boedecker, and C. Henkel, All-frequency effective medium theory of a photonic crystal, Opt. Express 11, 1590 (2003)

[17] Q. Zhao, J. Liu, D. Gao, K. You, X. Wang, H. M. Leung, T. K. Yung, R. Zhang, and W. Y. Tam, Reflection phase of photonic bands in finite bidirectional $1 D$ photonic crystals using an effective medium approach, OSA Continuum 1, 332 (2018)

[18] J. Liu, D. Gao, W. Mao, Q. Zhao, H. Ma, Y. Wang, X. Wang, T. K. Yung, and W. Y. Tam, Characterization of free-standing 1D photonic crystals using an effective medium approach, Opt. Lett. 44, 4853 (2019)

[19] T. H. Pei, and Y. T. Huang, The equivalent structure and some optical properties of the periodic-defect photonic crystal, J. Appl. Phys. 109, 073104 (2011)

[20] N. Bianchi, and L. Kahn, Topological Photonic States at a 1-D BinaryQuaternary Interface, (to be published) arXiv:1910.02920

[21] P. Markoš, and C. M. Soukoulis, Wave Propagation From Electrons to Photonic Crystals and Left-Handed Materials, (Princeton Unversity Press, 2008).

[22] A. Yariv, and P. Yeh, Optical Waves in Crystals: Propagation and Control of Laser Radiation, (John Wiley \& Sons, 1984).

[23] D. R. Smith, S. Schultz, P. Markos, and C. M. Soukoulis, Determination of effective permittivity and permeability of metamaterials from reflection and transmission coefficents, Phys. Rev. B 65, 195104 (2002)

[24] D. R. Smith, D. C. Vier, Th. Koschny, and C. M. Soukoulis, Electromagnetic parameters retrieval from inhomogeneous metamaterials, Phys. Rev. E 71, 036617 (2005)

[25] X. Chen, T. M Grzegorczyk, B. I. Wu, J. Pacheco Jr, and J. A. Kong, Robust method to retrieve the constitutive effective parameters of metamaterials, Phys. Rev. E 70, 016608 (2004)

[26] M. Notomi, Theory of light propagation in strongly modulated photonic crystals: Refractionlike behavior in the vicinity of the photonic band gap, Phys. Rev. B 62, 10696 (2000)

[27] J. P. Dowling, and C. M. Bowden, Anomalous index of refraction in photonic bandgap materials, J. Mod. Opt. 41, 345 (1994) 
[28] D. Y. Jeong, Y. H. Ye, and Q. M. Zhang, Effective optical properties associated with wave propagation in photonic crystals of finite length along the propagation direction, J. Appl. Phys. 92, 4194 (2002)

[29] N. Bianchi, and L. Kahn, Optical States in a 1-D Superlattice with Multiple Photonic Crystal Interfaces, (to be published) arXiv:2001.01165 


\section{APPENDIX}

\section{Transfer Matrix Method for general quaternary unit cell}

In this appendix, the dispersion relation for a PC with a general 4-layer unit cell is derived. The derivation follows a similar format to the binary unit cell, found in [1]. The direction of propagation is normal to the interfaces in the $+\hat{\mathbf{z}}$ direction, with $\mathbf{E}=E_{x} \hat{\mathbf{x}}$ and $\mathbf{H}=H_{y} \hat{\mathbf{y}}$, where:

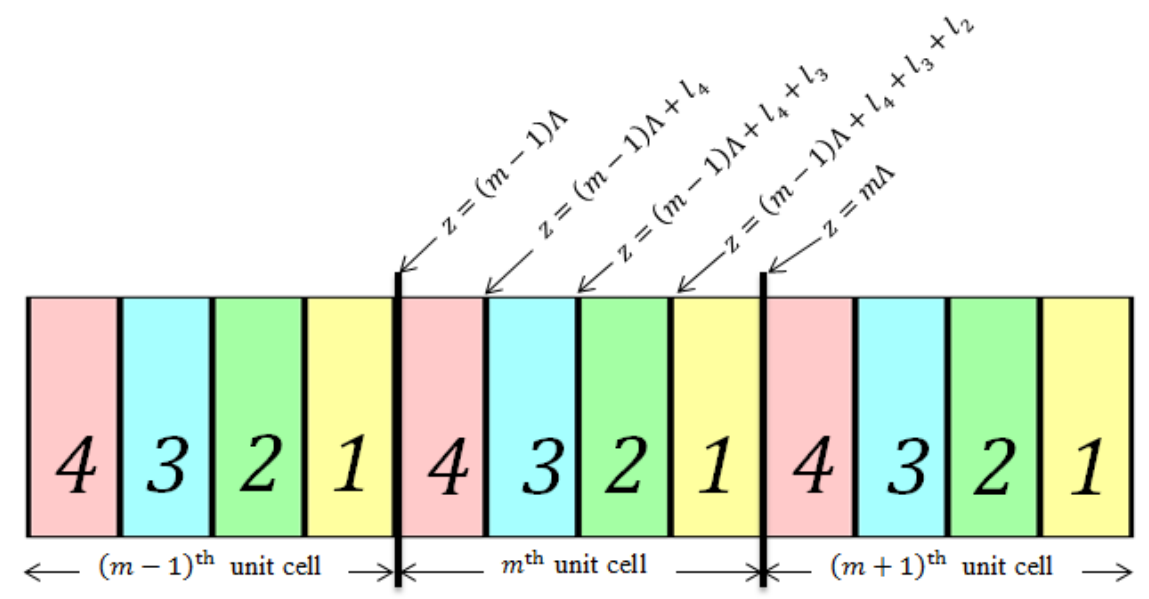

Figure A.1: Diagram of the PC used in the calculations below

$$
\begin{gathered}
E_{x}=a_{m}^{(\alpha)} e^{-i k_{\alpha}(z-m \Lambda)}+b_{m}^{(\alpha)} e^{i k_{\alpha}(z-m \Lambda)} \\
H_{y}=\frac{1}{i \omega \mu_{\alpha} \mu_{0}} \frac{\partial E_{x}}{\partial z}
\end{gathered}
$$

In Fig. A.1, the unit cell, $m$, is composed of layers, $\alpha=\{1,2,3,4\}$. In Eq. (A.2), $\omega$ is angular frequency, $\mu_{\alpha}$ is the relative permeability of layer $\alpha$, and $\mu_{0}$ is the free space permeability. Matching Eq. (A.1) and Eq. (A.2) across interface $z=$ $(m-1) \Lambda$, 


$$
\left(\begin{array}{cc}
1 & 1 \\
1 & -1
\end{array}\right)\left(\begin{array}{l}
a_{m-1}^{(1)} \\
b_{m-1}^{(1)}
\end{array}\right)=\left(\begin{array}{cc}
e^{i k_{4} \Lambda} & e^{-i k_{4} \Lambda} \\
\frac{z_{1}}{z_{4}} e^{i k_{4} \Lambda} & -\frac{z_{1}}{z_{4}} e^{-i k_{4} \Lambda}
\end{array}\right)\left(\begin{array}{l}
a_{m}^{(4)} \\
b_{m}^{(4)}
\end{array}\right)
$$

Simplifying Eq. (A.3) yields,

$$
\left(\begin{array}{l}
a_{m-1}^{(1)} \\
b_{m-1}^{(1)}
\end{array}\right)=\frac{1}{2}\left(\begin{array}{ll}
\left(1+\frac{z_{1}}{z_{4}}\right) e^{i k_{4} \Lambda} & \left(1-\frac{z_{1}}{z_{4}}\right) e^{-i k_{4} \Lambda} \\
\left(1-\frac{z_{1}}{z_{4}}\right) e^{i k_{4} \Lambda} & \left(1+\frac{z_{1}}{z_{4}}\right) e^{-i k_{4} \Lambda}
\end{array}\right)\left(\begin{array}{c}
a_{m}^{(4)} \\
b_{m}^{(4)}
\end{array}\right)
$$

Matching Eq. (A.1) and Eq. (A.2) across interface $z=(m-1) \Lambda+l_{4}$,

$$
\left(\begin{array}{cc}
e^{i k_{4}\left(\Lambda-l_{4}\right)} & e^{-i k_{4}\left(\Lambda-l_{4}\right)} \\
e^{i k_{4}\left(\Lambda-l_{4}\right)} & -e^{-i k_{4}\left(\Lambda-l_{4}\right)}
\end{array}\right)\left(\begin{array}{c}
a_{m}^{(4)} \\
b_{m}^{(4)}
\end{array}\right)=\left(\begin{array}{cc}
e^{i k_{3}\left(\Lambda-l_{4}\right)} & e^{-i k_{3}\left(\Lambda-l_{4}\right)} \\
\frac{z_{4}}{z_{3}} e^{i k_{3}\left(\Lambda-l_{4}\right)} & -\frac{z_{4}}{z_{3}} e^{-i k_{3}\left(\Lambda-l_{4}\right)}
\end{array}\right)\left(\begin{array}{c}
a_{m}^{(3)} \\
b_{m}^{(3)}
\end{array}\right)
$$

Simplifying Eq. (A.5) yields,

$$
\left(\begin{array}{c}
a_{m}^{(4)} \\
b_{m}^{(4)}
\end{array}\right)=\frac{1}{2}\left(\begin{array}{ll}
\left(1+\frac{z_{4}}{z_{3}}\right) e^{i\left(k_{3}-k_{4}\right)\left(\Lambda-l_{4}\right)} & \left(1-\frac{z_{4}}{z_{3}}\right) e^{-i\left(k_{3}+k_{4}\right)\left(\Lambda-l_{4}\right)} \\
\left(1-\frac{z_{4}}{z_{3}}\right) e^{i\left(k_{3}+k_{4}\right)\left(\Lambda-l_{4}\right)} & \left(1+\frac{z_{4}}{z_{3}}\right) e^{-i\left(k_{3}-k_{4}\right)\left(\Lambda-l_{4}\right)}
\end{array}\right)\left(\begin{array}{c}
a_{m}^{(3)} \\
b_{m}^{(3)}
\end{array}\right)
$$

Matching Eq. (A.1) and Eq. (A.2) across interface $z=(m-1) \Lambda+l_{4}+l_{3}$,

$$
\left(\begin{array}{cc}
e^{i k_{3}\left(l_{1}+l_{2}\right)} & e^{-i k_{3}\left(l_{1}+l_{2}\right)} \\
e^{i k_{3}\left(l_{1}+l_{2}\right)} & -e^{-i k_{3}\left(l_{1}+l_{2}\right)}
\end{array}\right)\left(\begin{array}{c}
a_{m}^{(3)} \\
b_{m}^{(3)}
\end{array}\right)=\left(\begin{array}{cc}
e^{i k_{2}\left(l_{1}+l_{2}\right)} & e^{-i k_{2}\left(l_{1}+l_{2}\right)} \\
\frac{z_{3}}{z_{2}} e^{i k_{2}\left(l_{1}+l_{2}\right)} & -\frac{z_{3}}{z_{2}} e^{-i k_{2}\left(l_{1}+l_{2}\right)}
\end{array}\right)\left(\begin{array}{c}
a_{m}^{(2)} \\
b_{m}^{(2)}
\end{array}\right)
$$

Simplifying Eq. (A.7) yields,

$$
\left(\begin{array}{c}
a_{m}^{(3)} \\
b_{m}^{(3)}
\end{array}\right)=\frac{1}{2}\left(\begin{array}{ll}
\left(1+\frac{z_{3}}{z_{2}}\right) e^{i\left(k_{2}-k_{3}\right)\left(l_{1}+l_{2}\right)} & \left(1-\frac{z_{3}}{z_{2}}\right) e^{-i\left(k_{2}+k_{3}\right)\left(l_{1}+l_{2}\right)} \\
\left(1-\frac{z_{3}}{z_{2}}\right) e^{i\left(k_{2}+k_{3}\right)\left(l_{1}+l_{2}\right)} & \left(1+\frac{z_{3}}{z_{2}}\right) e^{-i\left(k_{2}-k_{3}\right)\left(l_{1}+l_{2}\right)}
\end{array}\right)\left(\begin{array}{c}
a_{m}^{(2)} \\
b_{m}^{(2)}
\end{array}\right)
$$


Matching Eq. (A.1) and Eq. (A.2) across interface $z=(m-1) \Lambda+l_{4}+l_{3}+l_{2}$,

$$
\left(\begin{array}{cc}
e^{i k_{2} l_{1}} & e^{-i k_{2} l_{1}} \\
e^{i k_{2} l_{1}} & -e^{-i k_{2} l_{1}}
\end{array}\right)\left(\begin{array}{c}
a_{m}^{(2)} \\
b_{m}^{(2)}
\end{array}\right)=\left(\begin{array}{cc}
e^{i k_{1} l_{1}} & e^{-i k_{1} l_{1}} \\
\frac{z_{2}}{z_{1}} e^{i k_{1} l_{1}} & -\frac{z_{2}}{z_{1}} e^{-i k_{1} l_{1}}
\end{array}\right)\left(\begin{array}{c}
a_{m}^{(1)} \\
b_{m}^{(1)}
\end{array}\right)
$$

Simplifying Eq. (A.9) yields,

$$
\left(\begin{array}{c}
a_{m}^{(2)} \\
b_{m}^{(2)}
\end{array}\right)=\frac{1}{2}\left(\begin{array}{cc}
\left(1+\frac{z_{2}}{z_{1}}\right) e^{i\left(k_{1}-k_{2}\right) l_{1}} & \left(1-\frac{z_{2}}{z_{1}}\right) e^{-i\left(k_{1}+k_{2}\right) l_{1}} \\
\left(1-\frac{z_{2}}{z_{1}}\right) e^{i\left(k_{1}+k_{2}\right) l_{1}} & \left(1+\frac{z_{2}}{z_{1}}\right) e^{-i\left(k_{1}-k_{2}\right) l_{1}}
\end{array}\right)\left(\begin{array}{c}
a_{m}^{(1)} \\
b_{m}^{(1)}
\end{array}\right)
$$

Plugging Eq. (A.10) into the RHS of Eq. (A.8), Eq. (A.8) into the RHS of Eq. (A.6), and finally, Eq. (A.6) into the RHS of Eq. (A.4),

$$
\left(\begin{array}{l}
a_{m-1}^{(1)} \\
b_{m-1}^{(1)}
\end{array}\right)=\left(\begin{array}{ll}
t_{11} & t_{12} \\
t_{21} & t_{22}
\end{array}\right)\left(\begin{array}{l}
a_{m}^{(1)} \\
b_{m}^{(1)}
\end{array}\right)
$$

where the four matrix elements are given by,

$$
\begin{aligned}
t_{11} & =e^{i \phi_{1}}\left(\cos \phi_{2} \cos \phi_{3} \cos \phi_{4}+i z_{12}^{+} \sin \phi_{2} \cos \phi_{3} \cos \phi_{4}+i z_{13}^{+} \cos \phi_{2} \sin \phi_{3} \cos \phi_{4}\right. \\
& -z_{23}^{+} \sin \phi_{2} \sin \phi_{3} \cos \phi_{4}+i z_{14}^{+} \cos \phi_{2} \cos \phi_{3} \sin \phi_{4}-z_{24}^{+} \sin \phi_{2} \cos \phi_{3} \sin \phi_{4} \\
& \left.-z_{34}^{+} \cos \phi_{2} \sin \phi_{3} \sin \phi_{4}-i z_{1324}^{+} \sin \phi_{2} \sin \phi_{3} \sin \phi_{4}\right)
\end{aligned}
$$

$$
\begin{aligned}
t_{12} & =e^{-i \phi_{1}}\left(i z_{12}^{-} \sin \phi_{2} \cos \phi_{3} \cos \phi_{4}+i z_{13}^{-} \cos \phi_{2} \sin \phi_{3} \cos \phi_{4}+z_{23}^{-} \sin \phi_{2} \sin \phi_{3} \cos \phi_{4}\right. \\
& +i z_{14}^{-} \cos \phi_{2} \cos \phi_{3} \sin \phi_{4}+z_{24}^{-} \sin \phi_{2} \cos \phi_{3} \sin \phi_{4}+z_{34}^{-} \cos \phi_{2} \sin \phi_{3} \sin \phi_{4} \\
& \left.-i z_{1324}^{-} \sin \phi_{2} \sin \phi_{3} \sin \phi_{4}\right)
\end{aligned}
$$

$$
t_{21}=t_{12}^{*}
$$




$$
t_{22}=t_{11}^{*}
$$

where $z_{i j}$ is given by Eq. (13) and $z_{1324}$ is:

$$
z_{1324}^{ \pm}=\frac{1}{2}\left(\frac{z_{1} z_{3}}{z_{2} z_{4}} \pm \frac{z_{2} z_{4}}{z_{1} z_{3}}\right)
$$

In Eq. (A.12) and Eq. (A.13), $\phi_{i} \equiv k_{i} l_{i}$. The dispersion relation can be written using the equation $2 \cos (\kappa \Lambda)=t_{11}+t_{22}$ :

$$
\begin{aligned}
\cos (\kappa \Lambda) & =\cos \phi_{1} \cos \phi_{2} \cos \phi_{3} \cos \phi_{4}-z_{12}^{+} \sin \phi_{1} \sin \phi_{2} \cos \phi_{3} \cos \phi_{4} \\
& -z_{13}^{+} \sin \phi_{1} \cos \phi_{2} \sin \phi_{3} \cos \phi_{4}-z_{14}^{+} \sin \phi_{1} \cos \phi_{2} \cos \phi_{3} \sin \phi_{4} \\
& -z_{23}^{+} \cos \phi_{1} \sin \phi_{2} \sin \phi_{3} \cos \phi_{4}-z_{24}^{+} \cos \phi_{1} \sin \phi_{2} \cos \phi_{3} \sin \phi_{4} \\
& -z_{34}^{+} \cos \phi_{1} \cos \phi_{2} \sin \phi_{3} \sin \phi_{4}+z_{1324}^{+} \sin \phi_{1} \sin \phi_{2} \sin \phi_{3} \sin \phi_{4}
\end{aligned}
$$

Note that Eqs. (A.12) - (A.17) reduce to the expressions for the two and three layer unit cell when $\phi_{3}=\phi_{4}=0$ and $\phi_{4}=0$, respectively. If we let $1 \rightarrow A, 2 \rightarrow C$, $3 \rightarrow B$, and $4 \rightarrow C$, the dispersion relation reduces to Eq. (15). Alternatively, by using the product to sum identities:

$$
\begin{aligned}
\cos \phi_{i} \cos \phi_{j} & =\frac{1}{2}\left(\cos \left(\phi_{i}-\phi_{j}\right)+\cos \left(\phi_{i}+\phi_{j}\right)\right) \\
\sin \phi_{i} \sin \phi_{j} & =\frac{1}{2}\left(\cos \left(\phi_{i}-\phi_{j}\right)-\cos \left(\phi_{i}+\phi_{j}\right)\right)
\end{aligned}
$$

Eq. (A.17) can be written as: 


$$
\begin{aligned}
8 \cos (\kappa \Lambda) & =\left(1+z_{12}^{+}+z_{13}^{+}+z_{14}^{+}+z_{23}^{+}+z_{24}^{+}+z_{34}^{+}+z_{1324}^{+}\right) \cos \left(\phi_{1}+\phi_{2}+\phi_{3}+\phi_{4}\right) \\
& +\left(1-z_{12}^{+}+z_{13}^{+}+z_{14}^{+}-z_{23}^{+}-z_{24}^{+}+z_{34}^{+}-z_{1324}^{+}\right) \cos \left(\phi_{1}-\phi_{2}+\phi_{3}+\phi_{4}\right) \\
& +\left(1+z_{12}^{+}-z_{13}^{+}+z_{14}^{+}-z_{23}^{+}+z_{24}^{+}-z_{34}^{+}-z_{1324}^{+}\right) \cos \left(\phi_{1}+\phi_{2}-\phi_{3}+\phi_{4}\right) \\
& +\left(1+z_{12}^{+}+z_{13}^{+}-z_{14}^{+}+z_{23}^{+}-z_{24}^{+}-z_{34}^{+}-z_{1324}^{+}\right) \cos \left(\phi_{1}+\phi_{2}+\phi_{3}-\phi_{4}\right) \\
& +\left(1-z_{12}^{+}-z_{13}^{+}+z_{14}^{+}+z_{23}^{+}-z_{24}^{+}-z_{34}^{+}+z_{1324}^{+}\right) \cos \left(\phi_{1}-\phi_{2}-\phi_{3}+\phi_{4}\right) \\
& +\left(1-z_{12}^{+}+z_{13}^{+}-z_{14}^{+}-z_{23}^{+}+z_{24}^{+}-z_{34}^{+}+z_{1324}^{+}\right) \cos \left(\phi_{1}-\phi_{2}+\phi_{3}-\phi_{4}\right) \\
& +\left(1+z_{12}^{+}-z_{13}^{+}-z_{14}^{+}-z_{23}^{+}-z_{24}^{+}+z_{34}^{+}+z_{1324}^{+}\right) \cos \left(\phi_{1}+\phi_{2}-\phi_{3}-\phi_{4}\right) \\
& +\left(1-z_{12}^{+}-z_{13}^{+}-z_{14}^{+}+z_{23}^{+}+z_{24}^{+}+z_{34}^{+}-z_{1324}^{+}\right) \cos \left(\phi_{1}-\phi_{2}-\phi_{3}-\phi_{4}\right)
\end{aligned}
$$

\section{A.1 References \\ List of References}

[1] A. Yariv, and P. Yeh, Optical Waves in Crystals: Propagation and Control of Laser Radiation, (John Wiley \& Sons, 1984). 